
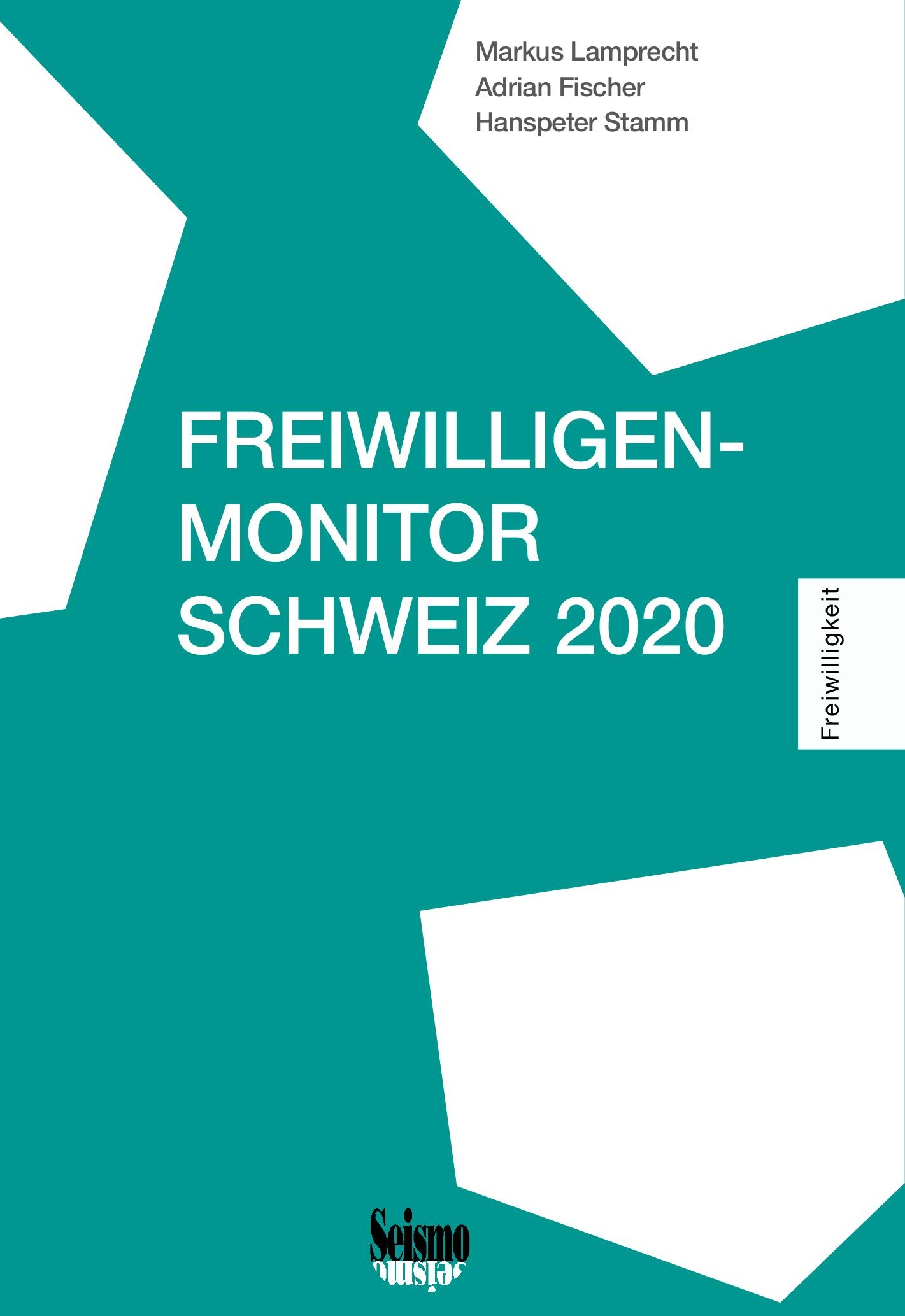
Markus Lamprecht, Adrian Fischer, Hanspeter Stamm

Freiwilligen-Monitor Schweiz 2020 
Die Reihe «Freiwilligkeit» im Seismo Verlag wird von der Schweizerischen Gemeinnützigen Gesellschaft (SGG) herausgegeben. Die Publikationen thematisieren Fragen zur Freiwilligenarbeit und zum zivilgesellschaftlichen Engagement in der Schweiz. Forschungsprojekte im Bereich Freiwilligenarbeit können jeweils bis zum 15. März bzw. 15. September bei der SGG eingereicht werden und werden von deren «Kommission Forschung Freiwilligkeit» (KFF) begleitet und zur Publikation vorgeschlagen.

\section{Mitglieder der KFF}

Peter Farago, Prof. Dr. (Präsident KFF), ehem. Direktor FORS in Lausanne Heinz Altorfer, Vize-Präsident der SGG, ehem. Leiter der Abt. Soziales bei Migros Kulturprozent

Jeannette Behringer, Dr., Fachstelle Gesellschaft \& Ethik der Ev.-ref. Landeskirche Zürich Sandro Cattacin, Prof. Dr., Directeur de I'Institut de recherches sociologiques, Université de Genève

Cornelia Hürzeler, Projektleitung Zivilgesellschaft beim Migros-Kulturprozent, Zürich Markus Lamprecht, Dr., Soziologe, Lamprecht \& Stamm Sozialforschung und Beratung Lukas Niederberger, Geschäftsleiter Schweizerische Gemeinnützige Gesellschaft (SGG) Jacqueline Schön-Bühlmann, Bundesamt für Statistik BFS, Neuchâtel Christian Staerklé, Prof. Dr. au Laboratoire de psychologie sociale, Université de Lausanne Muriel Surdez, Prof. Dr., Soziologin, Université de Fribourg

\section{Adresse zur Einreichung von Forschungsgesuchen im Bereich Freiwilligenarbeit}

Schweizerische Gemeinnützige Gesellschaft (SGG)

Schaffhauserstrasse 7 / Postfach 322

$\mathrm{CH}-8042$ Zürich

www.sgg-ssup.ch 
Markus Lamprecht

Adrian Fischer

Hanspeter Stamm

Freiwilligen-Monitor Schweiz 2020
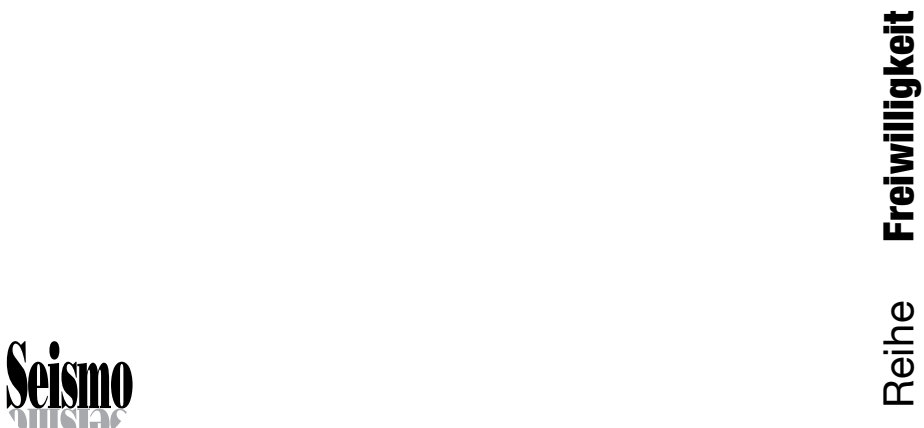

$\frac{\text { c }}{\frac{1}{(1)}}$ 
Publiziert von

Seismo Verlag, Sozialwissenschaften und Gesellschaftsfragen AG

Zürich und Genf

www.seismoverlag.ch

buch@seismoverlag.ch

Texte (C) 2020 Die Autoren

Umschlag: holenstein \& holenstein, visuelle kommunikation gmbh, Zürich

\section{BY NC ND Commons Namensnennung - Nicht kommerziell - Keine Bearbeitungen (CC BY-NC-ND 4.0) International Lizenz}

ISBN 978-3-03777-215-7 (Print)

ISBN 978-3-03777-733-6 (PDF)

http://doi.org/10.33058/seismo.30733

Der Seismo Verlag wird vom Bundesamt für Kultur

mit einer Förderprämie für die Jahre 2019-2020 unterstützt. 


\section{Inhaltsverzeichnis}

Vorwort $\quad 7$

Das Wichtigste in Kürze $\quad 9$

Einleitung

$1 \quad$ Freiwilligkeit $\quad \underline{19}$

1.1 Die verschiedenen Formen von Freiwilligkeit $\quad 21$

1.2 Die Veränderung des freiwilligen Engagements $\quad 29$

$2 \quad$ Formelle Freiwilligenarbeit und Ehrenamt $\quad \underline{37}$

2.1 Freiwilligenarbeit in Vereinen und Organisationen $\quad 39$

2.2 Das soziale Profil der Freiwilligen in Vereinen und Organisationen 46

2.3 Art der Tätigkeiten der Freiwilligen $\quad 51$

$3 \quad$ Informelle Freiwilligenarbeit $\quad \underline{61}$

3.1 Informelle Freiwilligentätigkeiten im eigentlichen und weiten Sinne 63

$\begin{array}{lll}3.2 & \text { Das soziale Profil der informell Freiwilligen } & 69\end{array}$

$4 \quad$ Spenden und weitere Formen von Freiwilligkeit $\quad \underline{77}$

4.1 Spendentätigkeit $\quad 79$

4.2 Das soziale Profil der Spenderinnen und Spender 81

4.3 Freiwilliges Engagement im Internet $\quad 85$

$\begin{array}{lll}4.4 & \text { Sharing Economy } & 88\end{array}$

$\underline{5} \quad$ Motive, Mobilisierung und Potenzial $\quad \underline{93}$

5.1 Motive und Zufriedenheit der formellen Freiwilligen $\quad 95$

5.2 Motive der informell Freiwilligen und der Spendenden $\quad 101$

5.3 Potenzial und Mobilisierung zukünftiger Freiwilliger $\quad 104$

5.4 Unterstützungsleistungen für die Freiwilligenarbeit $\quad 110$

$\underline{6} \quad$ Politisches Engagement, Vertrauen und die Freiwilligkeit $\quad \underline{117}$

Untersuchungsmethode und Stichprobe $\quad 127$

Literaturverzeichnis 135

Dank 143 



\section{Vorwort}

Liebe Leserin, lieber Leser

Die Entwicklung der Freiwilligenarbeit wird gerne reduziert auf wenige kurze Schlagzeilen:

> Freiwilligenarbeit nimmt in Vereinen drastisch ab.

> Freiwillige wollen zunehmend mitreden und mitbestimmen.

> Junge Freiwillige wollen flexibel und online wirken und Kompetenzen erwerben.

> Das Potenzial für mehr Freiwillige liegt bei AusländerInnen, Jungen und Personen über 6o Jahren.

Nimmt man sich allerdings Zeit, den Freiwilligen-Monitor mit seinen zahlreichen Tabellen und Grafiken genauer zu studieren, wird das Bild der Freiwilligenarbeit komplexer und differenzierter. Zwar sind nach wie vor mehr Männer als Frauen formell freiwillig in Vereinen tätig. Aber wenn man bedenkt, dass es sehr viel mehr Sportvereine als kulturelle und soziale Organisationen gibt, muss man diese Zahlen stark relativieren. Und in der informellen Freiwilligenarbeit, die mehrheitlich Care-Arbeit umfasst, sind viel mehr Frauen als Männer tätig. Schaut man aber auch hier genauer hin, stellt man fest, dass Männer, die zu weniger als 7o Prozent erwerbstätig sind, ebenso oft unbezahlt fürs Gemeinwohl wirken wie Frauen.

Der Freiwilligen-Monitor Schweiz 2020 hat drei Bereiche genauer untersucht: Erstens wurde nach den Gründen gefragt, warum Menschen nicht mehr oder noch nicht freiwillig tätig sind und unter welchen Umständen sie sich in bestimmten Bereichen freiwillig engagieren würden. Es wurde zweitens differenziert nach den von unbezahlten Engagements Begünstigten gefragt, um künftig Freiwilligenarbeit und Angehörigenbetreuung besser zuordnen zu können. Und drittens wurden die Formen finanzieller Vergütungen und Entschädigungen genauer erfragt, um künftig unbezahlte Freiwilligenarbeit und entschädigtes gemeinnütziges Engagement klarer unterscheiden zu können.

Gerade in der Corona-Krise haben Bundesämter und Kantone, Unternehmen und Schulen den Wert des freiwilligen Engagements stärker entdeckt und Freiwilligenarbeit als Quelle und Ausdruck einer lebendigen und solidarischen Gesellschaft erlebt. Wenn die verschiedenen gesellschaftlichen 
AkteurInnen das freiwillige Engagement vermehrt gemeinsam fördern, stehen die Karten gut, dass wir auch künftig gesellschaftlichen Herausforderungen mit innovativen Lösungen erfolgreich begegnen werden.

Eine anregende Lektüre wünscht Ihnen

Lukas Niederberger

Geschäftsleiter der SGG 


\section{Das Wichtigste in Kürze}

> Freiwilligkeit ist ein weites Feld. Der Freiwilligen-Monitor Schweiz zeigt nicht nur, wieviel Freiwilligenarbeit in der Schweiz geleistet wird. Er zeigt auch, dass es verschiedenste Formen von Engagement und unterschiedlichste Tätigkeitsbereiche gibt und dass sich Motive, Potenziale, Herausforderungen und Unterstützungsmassnahmen jeweils stark unterscheiden können.

> Die Schweizer Bevölkerung ist sehr engagiert: 39 Prozent der Bevölkerung im Alter ab 15 Jahren sind formell freiwillig innerhalb von Vereinen oder Organisationen tätig; 46 Prozent leisten informelle Freiwilligenarbeit, indem sie ausserhalb von Vereinen oder Organisationen Betreuungs- und Pflegearbeit leisten, anderen Personen beistehen oder bei Anlässen und Projekten mithelfen. Eine weitere Form von Freiwilligkeit ist das Spenden: 71 Prozent der Bevölkerung spenden Geld, 7 Prozent spenden Blut.

> Die Schweiz ist das Land der Vereine. Die hohe Zahl an Freiwilligen in den Vereinen und gemeinnützigen Organisationen erklärt sich auch durch die vielen Mitgliedschaften. Drei Viertel der Schweizer Bevölkerung im Alter ab 15 Jahren sind Mitglied in einem Verein oder einer gemeinnützigen Organisation. 61 Prozent der Bevölkerung machen dort aktiv mit. Am meisten Mitglieder zählen die Sportclubs vor den Spiel-, Hobby-und Freizeitvereinen, den kulturellen Vereinen und den Religionsgemeinschaften, Kirchen und kirchennahen Organisationen. Die Zahl der Freiwilligen bleibt erstaunlich stabil. In den vergangenen zehn Jahren kann weder ein markanter Rückgang noch ein klarer Anstieg von Freiwilligkeit festgestellt werden. Weder beim Spenden noch bei der formellen oder informellen Freiwilligenarbeit ist der Anteil an engagierten Personen eindeutig gesunken oder gestiegen. Was für die Freiwilligenarbeit im Allgemeinen gilt, gilt aber nicht mit Blick auf die verschiedenen Bereiche der Freiwilligenarbeit. Während man beim Sport, bei den Interessenverbänden und im öffentlichen Dienst eine Abnahme der formellen Freiwilligkeit beobachten kann, zählen wir in den Spiel-, Hobby- und Freizeitvereinen, in den kulturellen 
Vereinen sowie in den sozialen und karitativen Organisationen mehr Freiwillige.

> Das soziale Profil der formell Freiwilligen könnte sich in den nächsten Jahren verändern. Überproportional in Vereinen und gemeinnützigen Organisationen organisieren sich Männer, Personen im Alter von 45 bis 74 Jahren sowie BewohnerInnen auf dem Land und in der Deutschschweiz. Ein überdurchschnittliches Engagement zeigen auch Personen mit höherer Bildung und höherem Einkommen, Personen mit Schweizer Pass sowie Eltern mit Kindern im Alter von mehr als sechs Jahren. Das Potenzial scheint in diesen Bevölkerungsgruppen aber zunehmend ausgeschöpft zu sein. Frauen, Jüngere, Personen in der französisch-und italienischsprachigen Schweiz, StadtbewohnerInnen und in der Schweiz lebende Ausländerinnen und Ausländer zeigen eine hohe Bereitschaft, zukünftig mehr formelle Freiwilligenarbeit zu leisten. Obwohl Freiwilligenarbeit per definitionem unentgeltlich erbracht wird, wurden für den Freiwilligen-Monitor auch unterschiedliche Formen von gemeinnützigen Tätigkeiten erfragt, die finanziell vergütet oder materiell entschädigt werden. Am häufigsten erfolgt die Anerkennung für die geleistete Arbeit in Form von Essenseinladungen. Ein Fünftel der formell Freiwilligen erhält finanzielle Entschädigungen, die über Spesenentschädigungen hinausgehen. Männer werden eher finanziell entschädigt, und die Entschädigungen sind auch höher als bei den Frauen. Generell werden Ämter eher finanziell entschädigt, vor allem wenn es politische oder öffentliche Ämter betrifft. Freiwilligenarbeit mit einer finanziellen Entschädigung in Form von Honoraren, pauschalen Vergütungen oder Sitzungsgeldern gilt streng genommen nicht mehr als Freiwilligenarbeit, sondern als gemeinnütziges Engagement, selbst wenn es von Befragten als Freiwilligenarbeit empfunden und bezeichnet wird.

> Bei der informellen Freiwilligenarbeit handelt es sich oft um unbezahlte Care-Arbeit, und die Personen, die dabei betreut und gepflegt werden, sind häufig Verwandte oder Bekannte der Helfenden. Im Gegensatz zur formellen Freiwilligenarbeit wird informelle Freiwilligenarbeit häufiger von Frauen geleistet. Viele informell Freiwillige sind bereits pensioniert: Sie hüten einerseits ihre Enkelkinder und kümmern sich 
andererseits um ihre betagten Eltern. Solche Betreuungsarbeiten im familiären Umfeld können wegen bestehender moralischer Verpflichtungen streng genommen nicht als Freiwilligenarbeit gelten, selbst wenn die befragten Engagierten diese Dienste subjektiv als Freiwilligenarbeit empfinden und bezeichnen. Im Freiwilligen-Monitor wird deshalb zwischen informeller Freiwilligenarbeit im eigentlichen Sinne und informeller Freiwilligenarbeit im weiten Sinne unterschieden.

Weit verbreitet ist die Nachbarschaftshilfe. 72 Prozent der Bevölkerung im Alter ab 15 Jahren erbringen im Laufe eines Jahres kleine nachbarschaftliche Hilfeleistungen wie Aushelfen mit Kleinigkeiten, Briefkasten leeren oder Pflanzen giessen. Nachbarschaftshilfe ist in der Stadt genauso üblich wie auf dem Land.

Die Spendenbereitschaft und die Höhe der Spenden steigen mit dem Alter und dem verfügbaren Einkommen. Bezogen auf das jeweilige Einkommen sind GrossverdienerInnen aber nicht grosszügiger als Personen mit kleinem Lohn. Ältere Menschen spenden besonders häufig für junge Menschen und Kinder, für die Bekämpfung von Krankheiten und die Pflege von Kranken.

Das Internet und die Sharing Economy bieten neue, vielversprechende Formen von Freiwilligkeit. Bei den Internet-Freiwilligen handelt es sich häufig um junge, männliche Städter. Viel Internet-Freiwilligkeit geschieht im Rahmen der formellen Freiwilligentätigkeit in Vereinen und Organisationen. Immerhin ein gutes Drittel der InternetFreiwilligen engagiert sich aber ausschliesslich im Netz. Mit Blick auf die Sharing Economy zeigen nicht nur jüngere Personen eine hohe Bereitschaft, persönliche Dinge wie Bücher, Werkzeuge, das Auto oder gar die Wohnung zu teilen. Je persönlicher ein Gegenstand ist, desto weniger möchte man ihn allerdings teilen.

Wer sich formell freiwillig engagiert, will mit anderen Menschen etwas unternehmen und bewegen, ihnen helfen, sich dabei weiterentwickeln und seine Kenntnisse und Fähigkeiten erweitern sowie Spass haben. Die finanziellen Entschädigungen werden dagegen nur selten als Beweggrund genannt. Mehr als das Geld locken die Herausforderung, die Verantwortung und die Abwechslung. Die grosse Mehrheit der 
Ehrenamtlichen ist zufrieden mit ihrer Freiwilligentätigkeit und würde das Amt noch einmal übernehmen.

> Für die Beendigung eines freiwilligen Engagements sind in erster Line persönliche Gründe verantwortlich - insbesondere die Schwierigkeit, Beruf, Familie und Freiwilligentätigkeit unter einen Hut zu bringen. Neben der Zeitknappheit führen aber auch mangelnder Teamgeist, eine überhandnehmende Bürokratie oder fehlende Anerkennung zum Ausstieg.

> Obwohl sich schon viele in irgendeiner Form freiwillig engagieren, gibt es auch ungenutztes Potenzial. Die grundsätzliche Bereitschaft, sich neu bzw. erneut zu engagieren, ist vorhanden. Die Bedingungen für einen (Wieder-)Einstieg in die Freiwilligenarbeit sind: genügend Zeit, ein gutes Thema, Flexibilität und ein funktionierendes Team. Ein Engagement in sozialen und karitativen Organisationen sowie in Umwelt- und Tierschutzorganisationen scheint besonders viele NeueinsteigerInnen zu reizen.

> Bei der informellen Freiwilligenarbeit steht das Helfen im Zentrum. Es geht um soziale Kontakte - häufig auch zwischen den Generationen -, um das Gefühl gebraucht zu werden und etwas zurückzugeben. Es geht aber auch um die Erweiterung eigener Erfahrungen und Kenntnisse, um das Pflegen von Netzwerken, um Abwechslung und Spass. Recht viele Helfende würden sich bei ihren Betreuungs- und Pflegearbeiten mehr Unterstützung seitens anderer Angehöriger oder seitens des Staates wünschen.

> Freiwillige Tätigkeiten fördern das Vertrauen in andere Personen. Wer Freiwilligenarbeit leistet, hat deutlich mehr Vertrauen in seine Mitmenschen als diejenigen, die dies nicht tun. Besonders viel Vertrauen haben Personen, die in Vereinen und Organisationen freiwillig tätig sind. Freiwilligkeit wirkt sich auch auf das Vertrauen in die politischen Institutionen aus, nicht aber auf das Vertrauen in die Medien und die Wissenschaft.

> Die breite Darstellung von Freiwilligkeit und Freiwilligenarbeit im Monitor 2020 macht sichtbar, wie hoch das Engagement der Schweizer Bevölkerung ist und wie verschieden die Formen und die Motive des Engagements sein können. Gleichzeitig zeigt sich aber auch, dass 
die Grenzen zur Erwerbsarbeit auf der einen Seite sowie zur CareArbeit im familiären Nahraum auf der anderen Seite fliessend sind. Hier bedarf es einer vertieften Diskussion um die Kennzeichen und die zukünftige Erfassung der Freiwilligenarbeit. 



\section{Einleitung}

Eine Schweiz ohne Freiwilligenarbeit ist schwer vorstellbar. Bei der Besetzung politischer Ämter und Gremien, bei den Hilfswerken und Kirchen, in sozialen Bewegungen, bei vielen Freizeitaktivitäten, im Pflegebereich oder bei der Nachbarschaftshilfe: Überall spielt die Freiwilligenarbeit eine zentrale Rolle. Ohne freiwilliges Engagement würde eine tragende Säule unseres Gemeinwesens wegbrechen. Freiwilligenarbeit ist aber nicht nur für die Gesellschaft von unbezahlbarem Wert, sie kann auch für die Freiwilligen selbst ein grosser Gewinn sein. Freiwilligenarbeit bringt Menschen zusammen, schafft Kontakte und Freundschaften, erweitert den Horizont, stärkt das Selbstwertgefühl und eröffnet Partizipationschancen jenseits der Einbindung durch Erwerbsarbeit und Familie. In Freiwilligenorganisationen kann man nicht nur seine Kenntnisse und Fähigkeiten erweitern, man lernt in Vereinen die demokratischen Spielregeln, Solidarität und Kompromissbereitschaft und gewinnt Vertrauen in seine Mitmenschen und in die Institutionen.

Die Bedeutung der Freiwilligenarbeit für die Gesellschaft und die Freiwilligen untersucht der Freiwilligen-Monitor Schweiz. Der FreiwilligenMonitor ergänzt und vertieft seit 2007 die vom Bundesamt für Statistik im Rahmen der Schweizerischen Arbeitskräfteerhebung (SAKE) erhobenen Merkmale und Bedingungen der Freiwilligenarbeit um wichtige Kontextfaktoren zur Ausübung und zu vielen subjektiven Komponenten. Der Freiwilligen-Monitor fragt nicht nur, wo und in welchem Umfang sich die Bevölkerung freiwillig engagiert, sondern vor allem auch, weshalb und wie sie dies tut bzw. weshalb sie es nicht tut. Dank seiner grossen Stichprobe ermöglicht der Freiwilligen-Monitor differenzierte und zuverlässige Aussagen zu verschiedenen Arten der Freiwilligkeit. Er vermag Unterschiede zwischen verschiedenen Bevölkerungsgruppen und Regionen detailliert zu erfassen sowie Potenziale und Chancen zu erkennen. Der Freiwilligen-Monitor zeigt, wie vielfältig und facettenreich Freiwilligenarbeit ist und wie unterschiedlich sich die Problemlagen und Herausforderungen heute darstellen.

Der Freiwilligen-Monitor Schweiz wird seit Beginn von der Schweizerischen Gemeinnützigen Gesellschaft SGG herausgegeben. Zur Trägerschaft des Monitors gehören zudem das Migros-Kulturprozent, die Beisheim Stiftung sowie zo Partnerorganisationen. Der Freiwilligen-Monitor wird in enger 
Zusammenarbeit mit dem Bundesamt für Statistik realisiert. Die ersten drei Monitore (2007, 2010 und 2016) wurden vom Institut für Politikwissenschaft der Universität Bern unter Leitung von Markus Freitag durchgeführt. Er und sein Team legten den Grundstein für eine umfassende Dauerbeobachtung der Freiwilligenarbeit in der Schweiz. Der Freiwilligen-Monitor 2020 wurde von Lamprecht \& Stamm Sozialforschung und Beratung durchgeführt.

Der Freiwilligen-Monitor kann auf das Engagement und die Unterstützung vieler Personen zählen. Ein besonderer Dank geht an die Projektgruppe Monitor, die das Projekt in den verschiedenen Phasen begleitet und unterstützt hat. Unter der Leitung von Lukas Niederberger und Peter Fargo wirkten dabei folgende Personen mit: Heinz Altorfer, Jeannette Behringer, Sandro Cattacin, Cornelia Hürzeler, Patrizia Rezzoli, Jacqueline SchönBühlmann und Emmanuelle Sierro-Schenk. Für ihren tatkräftigen Support bei der Durchführung der Erhebung danken wir Susanne Graf vom LINK Institut in Luzern.

Der grösste Dank geht an die 5002 Personen, die sich freiwillig und unentgeltlich die Zeit genommen haben, den Fragebogen auszufüllen. Auf ihren Erfahrungen und Einschätzungen beruhen alle Zahlen im FreiwilligenMonitor. Wir hoffen, dass der Freiwilligen-Monitor nicht nur ein Monitor über die Freiwilligen, sondern auch ein Monitor für die Freiwilligen ist. 



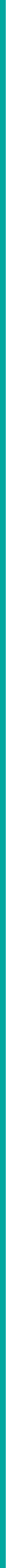




\section{Freiwilligkeit}

2 Formelle Freiwilligenarbeit

2 und Ehrenamt

3 Informelle Freiwilligenarbeit

4 Spenden und weitere Formen von Freiwilligkeit

5 Motive, Mobilisierung und Potenzial

Politisches Engagement, Vertrauen

6

und die Freiwilligkeit

Untersuchungsmethode und Stichprobe

Literaturverzeichnis 



\subsection{Die verschiedenen Formen von Freiwilligkeit}

\section{Was ist freiwilliges Engagement?}

Im Freiwilligen-Monitor wird bewusst eine breite Definition von Freiwilligkeit und freiwilligem Engagement verwendet. Dies geschieht mit der Absicht, möglichst die gesamte Bandbreite von freiwilligen, gemeinwohlförderlichen Leistungen zu erfassen (vgl. Ammann 2001; Ammann 2008, StadelmannSteffen et al. 2007; Stadelmann-Steffen et al. 2010; Freitag et al. 2016). Die Begriffsbestimmung im Freiwilligen-Monitor lehnt sich an die aus dem englischen Sprachraum stammende Bezeichnung Volunteering an. Unter Volunteering wird jede unbezahlte Aktivität verstanden, bei der Zeit oder Geld aufgewendet wird, um einer Person ausserhalb des eigenen Haushalts, einer Gruppe von Menschen oder einer Organisation zu nützen (Wilson 2ooo, 215). Anhand dieser Definition von Volunteering lassen sich drei Kriterien für freiwilliges Engagement benennen, die Freiwilligenarbeit von anderen gesellschaftlichen Bereichen unterscheiden.

1) Die Aktivität ist unbezahlt: Mit dem Kriterium der Unentgeltlichkeit grenzt sich Freiwilligenarbeit gegen die für Lohn und Bezahlung geleistete Erwerbsarbeit ab. Freiwilligenarbeit ist Nichterwerbsarbeit. Kleinere Aufwandentschädigungen kommen allerdings bei der Freiwilligenarbeit immer wieder vor. Wie hoch die Spesen und Vergütungen für ein Engagement sein dürfen, damit dieses noch als unentgeltliches Engagement gelten kann, und ab wann das Engagement zum finanziell vergüteten Einsatz wird, ist nicht eindeutig definiert.

2) Die Nutzniessenden sind Personen ausserhalb des eigenen Haushalts: Mit diesem Kriterium grenzt sich Freiwilligenarbeit von der Haus- und Familienarbeit sowie von Pflegeleistungen für Haushaltsangehörige ab. Dass diese Abgrenzung nicht ganz scharf und auch nicht ganz befriedigend ist, werden wir weiter unten noch sehen. 
3) Die Aktivität hat für andere Personen einen Nutzen: Eine Aktivität, die in erster Linie der handelnden Person nützt, ist kein freiwilliges Engagement. Ein guter Massstab dafür ist das sogenannte «Drittpersonen-Kriterium» (Bühlmann und Schmid 1999). Nach diesem Kriterium könnte die Aktivität an eine dritte Person gegen Bezahlung übertragen werden. Das heisst, die Aktivität muss für andere Personen oder Organisationen einen Wert erbringen, für den sie zu zahlen bereit wären. Wer einfach an einem Sporttraining teilnimmt, spendet keinen entsprechenden Nutzen. Wer das Training leitet und organisiert, aber sehr wohl. Das Sammeln von Unterschriften kann - wie Beispiele aus jüngerer Zeit zeigen - auch gegen Bezahlung an eine Organisation übertragen werden. Wer gratis Unterschriften sammelt, leistet Freiwilligenarbeit.

\section{Freiwillig und gemeinnützig}

Den drei Kriterien von Volunteering muss man noch hinzufügen, dass das Engagement nicht nur unentgeltlich, sondern eben auch freiwillig erfolgt. Freiwilligenarbeit geschieht aus freiem Willen und wird selbstbestimmt ohne rechtlichen Zwang ausgeübt (vgl. Nadai 2004). Dies unterscheidet sie - wie wir weiter unten sehen werden - von der Milizarbeit.

An Freiwilligenarbeit wird schliesslich der Anspruch gestellt, dass sie sich am Gemeinwohl orientiert und einen gemeinnützigen Beitrag leistet. In diesem Zusammenhang stellt sich die Frage, ab wann ein Verein als gemeinnützig gelten kann. Die Diskussion darüber, ob die Solidarität gemeinsamer Interessen, Lebenswelten und Freundschaften bereits ausreicht oder ob es eine über die eigene Gruppe hinausreichende Solidarität braucht, damit eine Freizeitaktivität zur Freiwilligenarbeit wird, ist im Monitor bewusst ausgeklammert. Die freiwillige und unentgeltlich Übernahme von Aufgaben in einem Verein zum Nutzen der anderen Vereinsmitglieder wird als Freiwilligenarbeit erfasst, unabhängig davon, als wie «gemeinnützig» man die Ziele des Vereins beurteilt.

Die Definition und Messung von Freiwilligkeit im Monitor orientiert sich auch an der Schweizerischen Arbeitskräfteerhebung (SAKE) des Bundesamtes für Statistik. Im Fragemodul «Unbezahlte Arbeit» werden darin seit 1997 in regelmässigen Zeitabständen Fragen zum freiwilligen Engagement gestellt. Der Freiwilligenmonitor wurde so konzipiert, dass sich die beiden 
Erhebungen ergänzen (Farago 2007, Ammann 2011). Entsprechend wurden Kernfragen im Freiwilligen-Monitor so gestellt, dass Vergleiche mit der SAKE möglich sind.

Je nachdem, in welchem Umfeld sie ausgeübt wird, lassen sich verschiedene Formen von Freiwilligkeit unterscheiden, die im Folgenden kurz erläutert werden. Wie wir sehen werden, hält sich die Wirklichkeit nur bedingt an unsere Definitionen und Vorgaben. Die Realität ist komplexer. Die Übergänge sind oft nicht scharf abgrenzbar, sondern fliessend.

\section{Freiwilligkeit: Freiwilligenarbeit und Spenden}

Aufder allgemeinsten und obersten begrifflichen Ebene steht die «Freiwilligkeit». Unter Freiwilligkeit werden alle Formen von Engagement verstanden, bei denen Personen eigene Ressourcen anderen Personen, Gruppen oder Organisationen freiwillig zur Verfügung stellen. Zur Freiwilligkeit gehört neben der Freiwilligenarbeit, die auf die Ressource Zeit zurückgreift, auch das Spenden, bei dem Geld, Naturalien oder Prestige eingebracht werden. Letzteres ist dann der Fall, wenn jemand seinen Namen für eine gute Sache zur Verfügung stellt. Wenngleich auch bei vielen Amtsübernahmen das Prestige des Amtsträgers oder der Amtsträgerin eine Rolle spielt und miteingebracht wird, ist das reine Spenden von Prestige doch relativ selten und wird im Freiwilligen-Monitor nicht erfasst. Es wird aber erfasst, wieviel Geld jemand spendet und an welche Form von Organisationen. Das Spenden von Naturalien kann von Schokolade im Rahmen der Aktion «2 X Weihnachten» über das Spenden von Kleidern oder Spielsachen bis zur Spende von Infrastruktur - zum Beispiel von sanitären Anlagen für ein Flüchtlingslager in einem Krisengebiet - reichen. Da hohe Sachspenden häufig an ihrem Geldwert gemessen werden, sind sie teilweise in den Geldspenden enthalten und werden nicht separat erfragt. Wir erfassen im Freiwilligen-Monitor aber eine wichtige Form von Naturalien-Spende - nämlich das Spenden von Blut. Spenden ist in der Schweizer Bevölkerung weit verbreitet. 71 Prozent der Schweizer Bevölkerung im Alter ab 15 Jahren haben im letzten Jahr Geld gespendet, 7 Prozent spendeten Blut. Spenden und freiwillige Tätigkeit zusammengezählt üben ganze 94 Prozent der Schweizer Bevölkerung eine Form von Freiwilligkeit aus (vgl. Abbildung 1.1). Mehr Informationen zum 
Spenden findet man in Kapitel 4, in dem wir uns auch näher mit der InternetFreiwilligkeit und der Sharing Economy befassen.

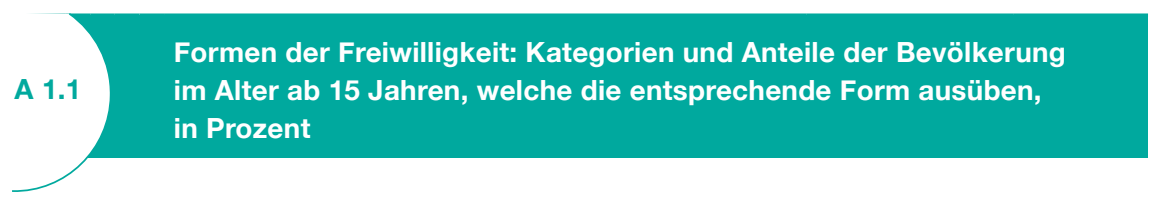

\section{Freiwilligkeit}

formelle und informelle Freiwilligenarbeit (inkl. gemeinnützige Engagements mit geringen Entschädigungen und unbezahlte Care-Arbeit für verwandte Personen ausserhalb des Haushalts), nachbarschaftliche Hilfeleistungen, Internet-Freiwilligkeit, Geld spenden, Blut spenden

Spenden von Geld, Naturalien und Prestige

\section{Geld spenden}

Blut spenden

Freiwilligenarbeit: formell und informell

inkl. gemeinnützige Engagements mit geringen Entschädigungen und unbezahlte Care-Arbeit für verwandte Personen ausserhalb des Haushalts

Formelle Freiwilligenarbeit

inkl. gemeinnützige Engagements mit geringen Entschädigungen

Formelle Freiwilligenarbeit ohne Amt

inkl. gemeinnützige Engagements mit geringen Entschädigungen

\section{Formelle Freiwilligenarbeit mit Amt/Ehrenamt}

inkl. gemeinnützige Engagements mit geringen Entschädigungen

Informelle Freiwilligenarbeit (im weiten Sinne)

inkl. unbezahlte Care-Arbeit für verwandte Personen ausserhalb des Haushalts

Informelle Freiwilligenarbeit im eigentlichen Sinne

ohne unbezahlte Care-Arbeit für verwandte Personen ausserhalb des Haushalts

\section{Sonderformen von Freiwilligkeit bzw. Freiwilligenarbeit}

Nachbarschaftliche Hilfeleistungen

Internet-Freiwilligkeit

Hinweise: Bei der formellen und der informellen Freiwilligenarbeit wurde nach der Ausübung in den vorangehenden vier Wochen gefragt, beim Spenden, den nachbarschaftlichen Hilfeleistungen und der Internet-Freiwilligkeit nach der Ausübung in den vorangehenden zwölf Monaten. Lesebeispiel: 39 Prozent der Bevölkerung leisten formelle Freiwilligenarbeit im Rahmen eines Vereins oder einer anderen Organisation. 16 Prozent haben dabei ein Amt inne, in welches sie gewählt oder berufen wurden (Ehrenamt). 23 Prozent engagieren sich in Vereinen oder anderen Organisationen ohne gewähltes Ehrenamt. 


\section{Freiwilligenarbeit: informell und formell}

Bei der Freiwilligenarbeit wird nicht Geld oder Blut gespendet, sondern Zeit. Neben Freiwilligenarbeit ist dabei auch von Freiwilligentätigkeit und freiwilligem Engagement die Rede. Wer Letzteres bevorzugt, will häufig die Differenz zur Erwerbsarbeit herausstreichen. Freiwilliges Engagement sei nicht nur nicht bezahlt, sondern auch nicht auf die Produktion von Gütern und Dienstleistungen in einem Betrieb ausgerichtet. Sowohl im englischen Begriff «Volunteering» als auch im französischen Begriff «bénévolat» wird ebenfalls der Aspekt der Freiwilligkeit betont, ohne eine Beziehung zu Arbeit herzustellen. Im Monitor wird keine Unterscheidung zwischen freiwilligem Engagement und Freiwilligenarbeit gemacht. Die Begriffe Freiwilligenarbeit, Freiwilligentätigkeit und freiwilliges Engagement werden synonym verwendet.

Formelle Freiwilligenarbeit erfolgt im Rahmen eines Vereins oder einer Organisation. Informelle Freiwilligenarbeit geschieht ausserhalb von Organisationen. Formelle Freiwilligenarbeit ist - vor allem wenn es sich um ein Amt handelt -zielgerichteter und geregelter; informelle Freiwilligenarbeit ist weniger organisiert, mehr privat als öffentlich und verläuft spontaner und individueller. Diese Unterscheidung ist in der Praxis aber nicht immer offensichtlich. So kann die gleiche Aktivität, je nachdem in welchem Kontext sie erfolgt, formelle oder informelle Freiwilligenarbeit sein. Wenn Sie an dem von Ihren NachbarInnen organisierten Grillfest Würste braten, handelt es sich um informelle Freiwilligenarbeit, wenn das Grillfest vom Quartierverein organisiert wurde, wird das Grillieren zur formellen Freiwilligenarbeit.

\section{Informelle Freiwilligenarbeit}

Da sie nicht im Rahmen einer Organisation, eines Vereins oder einer Institution und häufig spontaner erfolgt, ist die Erfassung von informeller Freiwilligenarbeit schwieriger. Im Monitor wird gefragt, ob man in den letzten vier Wochen unbezahlte Arbeit ausserhalb von Vereinen und Organisationen geleistet habe. Als Beispiele dafür werden Kinderhüten, Personen betreuen und pflegen, Transporthilfe, Nachbarschaftshilfe sowie die Mithilfe an Anlässen und Festlichkeiten angegeben. Entscheidend ist, dass sich diese Tätigkeiten - wie eingangs erwähnt - an Personen ausserhalb des eigenen Haushalts richten. Mit diesem Kriterium wird die informelle Freiwilligenar- 
beit von den Haus-, Betreuungs- und Pflegearbeiten innerhalb der engeren, unter einem Dach zusammenlebenden Familie abgegrenzt.

Für Pflege- und Betreuungsarbeiten wird heute auch häufig der englische Begriff Care-Arbeit verwendet, der neben der körperbezogenen Pflege auch das allgemeine «sich um eine Person kümmern» und entsprechende Unterstützungsleistungen einschliesst (Haberkern 2009). Während unbezahlte Care-Arbeit für Personen ausserhalb des eigenen Haushalts sowie ausserhalb des familiären Umfelds zur informellen Freiwilligenarbeit zählt, gehört Care-Arbeit für Haushaltsmitglieder und Verwandte nicht dazu (vgl. dazu auch Vogel und Tesch-Römer 2016). Neben dem Haushaltskriterium wurde im vorliegenden Monitor deshalb zusätzlich unterschieden, ob die informelle Tätigkeit für nächste Verwandte, andere Verwandte oder nichtverwandte Personen erfolgt. Wenn jemand sich auch für nichtverwandte Personen ausserhalb seines Haushalts engagiert, sprechen wir von informeller Freiwilligenarbeit im eigentlichen Sinne, da das Engagement über den familialen Nahraum hinausgeht und Nachbarinnen, Kollegen oder unbekannte Personen davon profitieren. Wenn wir zusätzlich zu dieser Freiwilligenarbeit im eigentlichen Sinne auch das Engagement von Personen berücksichtigen, die sich ausschliesslich um Verwandte ausserhalb des Haushaltes kümmern, so sprechen wir von Freiwilligenarbeit im weiten Sinne.

Informelle Freiwilligenarbeit im eigentlichen Sinne leisteten in den vier Wochen vor der Befragung 35 Prozent der Bevölkerung im Alter ab 15 Jahren, Freiwilligenarbeit im weiten Sinne insgesamt 46 Prozent. In den bisherigen Freiwilligen-Monitoren wurde diese Unterscheidung nicht gemacht. Informelle Freiwilligenarbeit entspricht dort unserer Definition von informeller Freiwilligenarbeit im weiten Sinne.

Wie sehr die Antworten und die entsprechenden Prozentwerte nicht nur vom Kreis der Nutzniessenden, sondern auch von Art und Umfang der Hilfeleistungen abhängig sind, zeigt sich daran, dass wir deutlich höhere Prozentwerte erhalten, wenn wir konkret nach nachbarschaftlichen Hilfeleistungen fragen. Die Frage: «Haben Sie in den letzten zwölf Monaten kleine nachbarschaftliche Hilfeleistungen erbracht (wie z. B. Aushelfen mit Kleinigkeiten, Briefkasten leeren, Pakete in Empfang nehmen, Pflanzen giessen oder Haustiere füttern?» beantworten 72 Prozent der Befragten mit «Ja». Im kleinen Rahmen und über einen längeren Zeithorizont engagieren 
sich also noch deutlich mehr Personen. Diese kleinen nachbarschaftlichen Hilfeleistungen zählen wir aber nicht zur informellen Freiwilligenarbeit. Genaueres dazu steht in Kapitel 3.

\section{Formelle Freiwilligenarbeit mit und ohne Amt}

Formelle Freiwilligenarbeit leistet man innerhalb von Vereinen und anderen Organisationen. Dabei kann zwischen dem Ehrenamt und anderen freiwilligen Tätigkeiten unterschieden werden. Der Unterschied besteht darin, dass man in ein Ehrenamt gewählt oder berufen wird, während es sich bei den anderen Tätigkeiten eher um Helferdienste handelt, die weniger verbindlich und verpflichtend sind. Ein Amt ist mit bestimmten Pflichten verbunden, die Aufgaben sind klarer definiert und die Verpflichtung erfolgt über einen längeren Zeithorizont, während Helferdienste häufig an einen konkreten Anlass geknüpft sind.

Zur Erfassung von formeller Freiwilligenarbeit wird im Monitor nach ehrenamtlichen oder freiwilligen Tätigkeiten gefragt, die man für einen Verein, für eine Organisation oder eine öffentliche Institution in den letzten vier Wochen vor der Befragung ausgeübt hat. Die Aufgaben und Arbeiten müssen freiwillig übernommen und unbezahlt oder gegen eine geringe Aufwandentschädigung geleistet werden. Zusätzlich wird unterschieden, ob es sich bei dieser Tätigkeit um ein gewähltes Amt handelt oder nicht. Ein gewähltes Amt haben 16 Prozent der Schweizer Bevölkerung übernommen. 23 Prozent sind ohne Amt formell freiwillig tätig. Insgesamt üben 39 Prozent der Schweizer Bevölkerung eine formelle Freiwilligentätigkeit oder ein gering entschädigtes gemeinnütziges Engagement aus. Wer wo und warum formell freiwillig tätig ist, lässt sich den Kapiteln 2 und 5 entnehmen.

\section{Milizarbeit im Freiwilligen-Monitor}

Milizarbeit bezeichnet die freiwillige, nebenberufliche und ehrenamtliche Übernahme von öffentlichen Aufgaben und Ämtern (vgl. Linder 2012, 82). Miliztätigkeit ist nicht Erwerbsarbeit, sie ist aber auch nicht deckungsgleich mit Freiwilligenarbeit. Linder spricht von Freiwilligenarbeit im erweiterten Sinne und Nadai bezeichnet das Milizamt als Spezial- oder Grenzfall des 
Ehrenamts (Nadai 2004). In der Schweiz hat Milizarbeit eine lange Tradition und erfüllt wichtige Funktionen für Gesellschaft und Politik. Verschiedene nationale und vor allem auch kommunale Aufgaben wie die Verteidigung oder die Feuerwehr sowie viele Mandate, Ämter und Aufgaben in Politik, Kirche oder Schule wurden und werden milizmässig erbracht. Der Unterschied zur eigentlichen Freiwilligenarbeit besteht darin, dass Milizarbeit eine gesetzliche Grundlage kennt und es strenggenommen eine Verpflichtung zur Milizarbeit gibt (Ammann 2008). Diese Verpflichtung betrifft immer noch die Dienstpflicht in der Armee. Wer der Dienstpflicht nicht nachkommt, kann heute einen Ersatzdienst leisten. Eine Verweigerung zieht Sanktionen nach sich. In vielen anderen Bereichen hat eine Ablehnung von Milizarbeit keine rechtlichen Folgen mehr. Hier ersetzen finanzielle Anreize die Verpflichtung. Die Milizarbeit wird in Form von Pauschalen oder Tag- und Sitzungsgeldern entschädigt und wird häufig zur Nebenbeschäftigung. In den letzten Jahren ist allerdings die Idee eines obligatorischen Bürgerdienstes diskutiert worden, mit dem das Milizsystem gerettet werden soll (zur Milizpolitik vgl. Müller 2015).

Milizarbeit wurde bei der Konzeption des Freiwilligen-Monitors deshalb nicht zur Freiwilligenarbeit gezählt, weil sie genau genommen nicht freiwillig und heute häufig auch nicht mehr unentgeltlich ist. Dass der Monitor die Milizarbeit nicht erfasst, wie dies manchmal moniert wird, ist allerdings nicht korrekt. Wer sein Milizamt als ehrenamtliche Tätigkeit für einen Verein, eine Organisation oder eine öffentliche Institution versteht, die er freiwillig übernommen hat und unbezahlt oder gegen eine geringe Entschädigung ausübt, wird im Monitor sehr wohl erfasst. Bei der Abfrage nach der Organisation und Art der Tätigkeit werden deshalb auch die typischen Felder für Milizarbeit erfragt wie «öffentlicher Dienst» oder «politisches oder öffentliches Amt oder Gremium». Milizarbeit wird im Freiwilligen-Monitor also dann erfasst, wenn sie freiwillig und weitgehend unentgeltlich ist. Der Einsatz in der freiwilligen Feuerwehr gehört da dazu, ein Nationalratsmandat aber ebenso wenig wie der Dienst in der Armee. 


\subsection{Die Veränderung des freiwilligen}

\section{Engagements}

\section{Die These vom fortschreitenden Verfall}

Freiwilligenarbeit ist für die Schweizer Gesellschaft von unbezahlbarem Wert, und sie hat hierzulande eine lange Tradition (Degen 2010; Schumacher 2010). Viele Aufgaben der öffentlichen Dienste, aber auch die meisten Aktivitäten von Vereinen und anderen nichtstaatlichen Organisationen sowie die meisten Unterstützungs- und Hilfeleistungen im sozialen Umfeld wären ohne Freiwillige undenkbar. Zu Recht wird Freiwilligenarbeit als unverzichtbare Ressource für das Gemeinwesen und die Gesellschaft gesehen. Gerade wegen ihrer grossen Bedeutung schwingt im Nachdenken über freiwilliges Engagement auch häufig die Sorge mit, Freiwilligenarbeit könnte erodieren und zurückgehen. Eine der prominentesten Stimmen dieser pessimistischen Einschätzung ist der amerikanische Soziologe Robert Putnam. In seinem vielzitierten Buch «Bowling Alone» beschreibt Putnam (1995, 2000) den Verlust an sozialen Bindungen und den Niedergang des freiwilligen Engagements in der amerikanischen Gesellschaft. Als Ursachen dafür nennt Putnam die Verknappung von Zeit und Geld in einer immer erfolgs- und leistungsorientierteren Gesellschaft, die Verstädterung, die langen Arbeitswege sowie das Fernsehen und die elektronischen Medien im Allgemeinen.

\section{Rückgang oder Verlagerung der Freiwilligenarbeit?}

Die Konsequenzen daraus sind nach Putnam dramatisch: Mit dem Verlust an Gemeinsinn und sozialem Engagement droht der Zerfall der Gesellschaft. Sicherheit, Gesundheit und wirtschaftliche Prosperität nehmen ab, während Kriminalität, Drogenkonsum und Arbeitslosigkeit zunehmen. Putnams Krisenszenario ist nicht unumstritten. So wird kritisiert, Putnam richte seinen Blick zu stark auf traditionelle Formen des sozialen Engagements und übersehe dabei die neu entstandenen Gemeinschaften und Netzwerke. Verschiedene ForscherInnen - gerade auch in der Schweiz und in Deutschland - sehen keinen generellen Schwund der Freiwilligenarbeit, sondern 
eher eine Verlagerung weg von traditionellen Formen des Engagements hin zu neuen Formen und Feldern (für Deutschland: z. B. Priller et al. 2011; für die Schweiz: z. B. Samochowiec, Thalmann und Müller 2018).

Angesichts der Brisanz dieser Diskussion über die Zukunft des freiwilligen Engagements, des sozialen Zusammenhalts und der Zivilgesellschaft ist es nicht erstaunlich, dass die erste Frage, die an den Monitor gestellt wird, stets lautet: Wie entwickelt sich die Freiwilligenarbeit? Nimmt sie ab oder zu? Von einem Monitor wird erwartet, dass er Entwicklungen über die Zeit aufzeigt. So wichtig und offensichtlich die Frage, so kompliziert und schwierig ist die Antwort. Eine Antwort auf die Frage nach den Veränderungen bei der Freiwilligenarbeit ist nicht möglich ohne einen kleinen Exkurs über Erhebungsmethoden.

\section{Die Veränderung der Erhebungsmethoden im Freiwilligen-Monitor}

Bei jedem neuen Freiwilligen-Monitor nimmt man Anpassungen vor. Man lernt aus den Erfahrungen, stellt Fragen und Filtern leicht um oder passt die Frageformulierungen an. Dies geschieht immer in bester Absicht. Man will die Befragung verbessern, die Kategorien schärfen oder neue Fragestellungen aufnehmen. Die kleinen Anpassungen können aber einen grossen Einfluss auf das Ergebnis haben.

Hinzu kommt, dass auch die Erhebungsmethode angepasst werden musste. Die Freiwilligen-Monitore 2007 und 2010 (Erhebungsjahre 2006 und 2009) wurden als telefonische Befragung (CATI: Computer Assisted Telephone Interviews) durchgeführt. Vor dem Hintergrund des technologischen Wandels im Telekommunikationsbereich und den Veränderungen im Kommunikationsverhalten der Bevölkerung wurden die Daten des Freiwilligen-Monitors 2016 (Erhebungsjahr 2014) über zwei Wege erhoben (Mixed-Mode). Die kontaktierten Personen konnten die Fragen entweder telefonisch beantworten (CATI) oder den Fragebogen online ausfüllen (CAWI: Computer Assisted Web Interviews). Etwa zwei Drittel der Interviews wurden telefonisch realisiert und ein Drittel online (vgl. Freitag et al. 2016, 38). Für den vorliegenden Freiwilligen-Monitor 2020 (Erhebungsjahr 2019) wurde die Erhebungsmethode erneut angepasst (vgl. Kapitel 7). Die Befragten konnten den Fragebogen 
online (CAWI) am Computer, Tablet oder Mobiltelefon beantworten oder sie konnten einen Fragebogen auf Papier ausfüllen (PAPI), der ihnen nach Hause geschickt wurde. Aufgrund der geänderten Erhebungsmethode mussten Anpassungen am Fragebogen vorgenommen werden.

\section{Wie Erhebungsmethoden die Ergebnisse beeinflussen können}

Mit der Anpassung der Erhebungsmethode sollte der sinkenden Erreichbarkeit und Teilnahmebereitschaft bei telefonischen Befragungen entgegengewirkt werden. Die Absicht war, eine möglichst hohe Ausschöpfung zu erreichen. Immer weniger Personen in der Schweiz haben einen Festnetzanschluss mit einer registrierten Telefonnummer. Vor allem junge Personen nutzen oft nur noch Mobiltelefone ohne eingetragene Telefonnummer. Zudem sind HandynutzerInnen weniger bereit, an langen telefonischen Befragungen teilzunehmen. Gleichzeitig steigt die Zahl der Internetnutzenden und die Bereitschaft, Fragebogen am Bildschirm auszufüllen. Das Problem ist nun, dass die Hürden, an einer Befragung teilzunehmen, für die verschiedenen Erhebungsarten unterschiedlich hoch sind: An Online-Befragungen (CAWI) nehmen häufiger Personen teil, die einen höheren Bildungsgrad haben und besonders engagiert bzw. besonders stark vom Thema angesprochen sind (vgl. Jacob et al. 2019). Beim Telefoninterview (CATI) ist dies weniger der Fall. Wenn wir im Folgenden die Befunde über die Zeit vergleichen, müssen wir die Auswirkungen dieser methodischen Anpassungen berücksichtigen.

\section{Wie verändert sich das freiwillige Engagement im Monitor?}

Betrachten wir in Tabelle 1.1 zunächst den Verlauf beim Spenden. Zwischen 2006 und 2009 sowie zwischen 2014 und 2019 bleibt der Anteil an Personen, die in der Schweiz für einen guten Zweck Geld spenden, praktisch unverändert. Eine signifikante Veränderung zeigt sich aber zwischen 2009 und 2014. Der Anteil an Spendenden geht um 5 Prozentpunkte von 76 auf 71 Prozent zurück. Diese Veränderung erklärt sich zumindest teilweise dadurch, dass in den ersten beiden Monitoren die Erfassung des Spendens zusammen mit 
dem Vereinsengagement gestützt nach Bereichen (Sport, Kultur, Soziales etc.) erfolgte, und in den beiden späteren Monitoren davon losgelöst, anhand einer allgemeinen Frage zum Spenden (vgl. Freitag et al. 2016, 106).

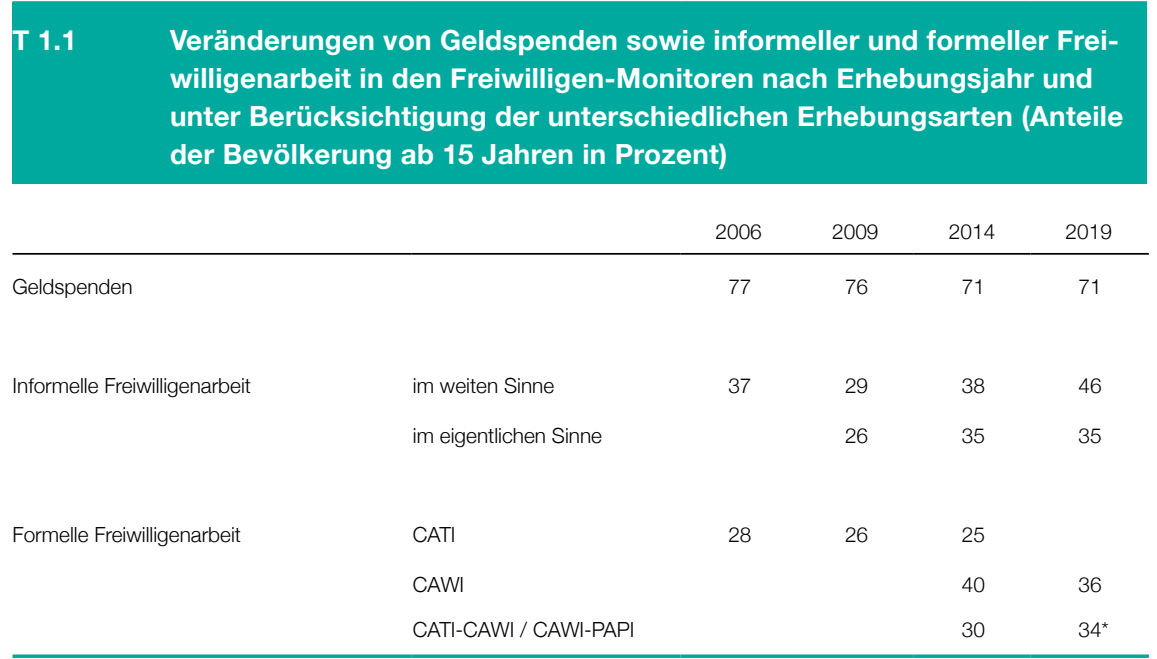

Abkürzungen: CATI: telefonische Befragung (Computer Assisted Telephone Interview); CAWI: schriftliche Online-Befragung (Computer Assisted Web Interview); PAPI: schriftliche Befragung mit Papierfragebogen (Paper-And-Pencil Interview). Hinweis: * Für die Vergleichbarkeit mit den Monitoren 2006 und 2009 mussten die Werte für 2014 und 2019 analog zur damaligen Erfassung berechnet werden. Der Wert für 2019 unterscheiden sich deshalb von dem in Abbildung 1.1 angegebenen Wert.

Bei der informellen Freiwilligenarbeit wird 2019 erstmals zwischen informeller Freiwilligenarbeit im eigentlichen Sinne und informeller Freiwilligenarbeit im weiten Sinne unterschieden. Bei der informellen Freiwilligenarbeit im eigentlichen Sinne engagiert man sich für nichtverwandte Personen ausserhalb des eigenen Haushalts. Bei der informellen Freiwilligenarbeit im weiten Sinne werden auch diejenigen Freiwilligen mitgezählt, deren Engagement sich auf verwandte Personen beschränkt (z. B. das Hüten von Grosskindern). Die informelle Freiwilligenarbeit im eigentlichen Sinne kann nachträglich auch für die Erhebungen 2009 und 2014 berechnet werden und lässt sich gut mit dem aktuellen Monitor vergleichen. Wie Tabelle 1.1 zeigt, gibt es in jüngster Zeit keine Veränderung. Der Anteil an Personen, die sich für nicht verwandte Personen ausserhalb des eigenen Haushalts engagieren, bleibt zwischen 2014 und 2019 stabil. Etwas irritierend ist der vorübergehende Einbruch der in- 
formellen Freiwilligenarbeit im Jahr 2009. Dafür findet sich keine einfache Erklärung. Wir vermuten, dass der Einbruch mit der unscharfen Abgrenzung zwischen informeller Freiwilligenarbeit und Haus- und Familienarbeit zusammenhängen könnte (vgl. auch Freitag et al. 2016, 77).

Bei der Veränderung der formellen Freiwilligenarbeit tritt der besagte Effekt der Erhebungsart am deutlichsten zu Tage. So kommt man in den telefonischen Interviews (CATI) aufgrund einer anderen Stichprobenzusammensetzung auf einen tieferen Anteil an formell Freiwilligen als in den schriftlichen Befragungen (CAWI, PAPI). Entsprechend messen wir den höchsten Anteil an Personen, die formelle Freiwilligenarbeit verrichten, 2014 und 2019 in der Online-Befragung (CAWI), den tiefsten in den telefonischen Befragungen (CATI) von 2009 und 2014. Wenn wir nur die telefonischen Erhebungen vergleichen, messen wir einen leichten Rückgang der formellen Freiwilligenarbeit zwischen 2006 und 2014 (vgl. Freitag et al. 2016). Wenn wir zwischen 2014 und 2019 nur die Online-Befragungen vergleichen, nimmt die formelle Freiwilligenarbeit ebenfalls ab, die Werte sind aber deut lich höher als in den telefonischen Befragungen. Wenn wir alle Befragten von 2014 (CATI und CAWI) mit allen Befragten von 2019 (CAWI und PAPI) vergleichen, so nimmt die formelle Freiwilligenarbeit dagegen um 4 Prozentpunkte zu.

Zusammenfassend lässt sich sagen, dass die Veränderungen über die Zeit im Monitor nicht abschliessend bestimmt werden können. Unter Berücksichtigung der verschiedenen Methodeneffekte ist aber doch augenfällig, wie wenig sich eigentlich ändert. Die Veränderungen in Tabelle 1.1 gehen immer mit einem Methodenwechsel einher.

\section{Wie verändert sich das freiwillige Engagement in der SAKE?}

Um die Ergebnisse im Monitor besser einordnen und beurteilen zu können, lohnt sich der Vergleich mit den Werten der Schweizerischen Arbeitskräfteerhebung (SAKE) des Bundesamtes für Statistik. Im Fragemodul «Unbezahlte Arbeit» werden seit 1997 Fragen zum freiwilligen Engagement gestellt, die mit den Fragen des Freiwilligen-Monitors vergleichbar sind (vgl. Abschnitt 1.1). In Tabelle 1.2 fällt zunächst auf, dass die SAKE tiefere Werte zur formellen und informellen Freiwilligenarbeit ausweist als der Monitor. Für die tieferen 
Werte können mehrere Gründe angegeben werden: Zunächst ist das Hauptziel der SAKE die Erfassung der Erwerbsstruktur und des Erwerbsverhaltens der Bevölkerung. Unbezahlte Arbeit und Freiwilligenarbeit machen nur einen kleinen Teil der Fragen aus. Zudem ist die SAKE eine Bundeserhebung, die mit grossem Aufwand als telefonische Befragung (CATI) durchgeführt wird. Aufgrund der breiteren Ausrichtung, einem Bundesamt als Absender und der aufwendigen telefonischen Rekrutierung erreicht die SAKE eine höhere Teilnahmebereitschaft, die vermehrt auch Personen mit tieferer Bildung und geringerem Engagement erfasst.

\begin{tabular}{|c|c|c|c|c|c|c|c|c|}
\hline \multirow[t]{2}{*}{ T 1.2} & \multicolumn{8}{|c|}{$\begin{array}{l}\text { Veränderungen von informeller und formeller Freiwilligenarbeit in der } \\
\text { Schweizerischen Arbeitskräfteerhebung (SAKE) des Bundesamtes für } \\
\text { Statistik nach Erhebungsjahr (Anteile der Bevölkerung ab } 15 \text { Jahren in } \\
\text { Prozent) }\end{array}$} \\
\hline & & 1997 & 2000 & 2004 & 2007 & 2010 & 2013 & 2016 \\
\hline \multicolumn{2}{|c|}{ Informelle Freiwilligenarbeit } & 30 & 23 & 23 & 21 & 18 & 18 & 32 \\
\hline \multicolumn{2}{|c|}{ Formelle Freiwilligenarbeit } & 27 & 25 & 25 & 24 & 20 & 20 & 20 \\
\hline
\end{tabular}

Hinweise: 2010 fand bei der SAKE ein Systemwechsel statt. Neu wurde die Erhebung über das ganze Kalenderjahr durchgeführt (vorher jeweils im zweiten Quartal des Jahres). Längere Zeitvergleiche sind deshalb nur bedingt möglich. 1997 wurde die informelle Freiwilligenarbeit ohne definierten Zeitraum («in den letzten 4 Wochen») erfasst. 2016 wurden die Fragen zur informellen Freiwilligenarbeit überarbeitet.

Bei den Veränderungen über die Zeit stellen wir Folgendes fest: Die formelle Freiwilligenarbeit nimmt zwischen 1997 und 2010 kontinuierlich ab. Zwischen 2010 und 2016 bleiben die Zahlen aber stabil. Bei der informellen Freiwilligenarbeit beobachten wir zwei grössere Sprünge zwischen 1997 und 2000 sowie zwischen 2013 und 2016, die sich jedoch hauptsächlich durch die Veränderung der Frage im Fragebogen erklären lassen. 1997 wurde gefragt, ob eine Person ab und zu unbezahlte Arbeiten wie z. B. fremde Kinder hüten, Nachbarschaftshilfe oder Transportdienste leiste. Ab 2000 wurde gefragt, ob sie dies in den letzten 4 Wochen geleistet habe. Für die Erhebung 2016 wurde die Frage umformuliert und einerseits das Adressatenfeld (Verwandte, Bekannte, Nachbarn) aufgeführt, sowie andererseits die Beispielaktivitäten erweitert (Kinder hüten, Pflege und andere Hilfeleistungen z. B. Hausarbeiten, Einkaufen, Transportdienste, Betreuung von Haustieren, Gartenarbeiten). 


\section{Es braucht eine differenziertere Betrachtung}

Aufgrund der Ergebnisse im Freiwilligen-Monitor und in der Schweizerischen Arbeitskräfteerhebung (SAKE) kann man folgendes Fazit ziehen: Ein genereller Rückgang von Freiwilligkeit kann in der Schweiz nicht beobachtet werden. Weder beim Spenden noch bei der informellen oder formellen Freiwilligenarbeit ist es in den letzten Jahren zu einem klaren Einbruch bei den freiwillig engagierten Personen gekommen. Dies will aber nicht heissen, dass es keine Veränderungen gibt. Um die Verschiebungen, aber auch die Probleme und Potenziale besser sichtbar machen zu können, braucht es eine differenziertere Betrachtung, die wir in den nächsten fünf Kapitel leisten wollen. 


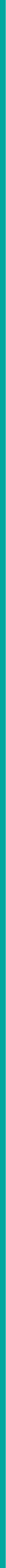


1 Freiwilligkeit

2 Formelle Freiwilligenarbeit und Ehrenamt

3 Informelle Freiwilligenarbeit

4 Spenden und weitere Formen von Freiwilligkeit

5 Motive, Mobilisierung und Potenzial

6

Politisches Engagement, Vertrauen und die Freiwilligkeit

Untersuchungsmethode und Stichprobe

Literaturverzeichnis 



\subsection{Freiwilligenarbeit in Vereinen und Organisationen}

\section{Verschiedene Formen des Engagements}

Formelle Freiwilligenarbeit wird innerhalb von Vereinen und Organisationen geleistet. In Vereinen und Organisationen kann man unterschiedlich mitmachen - nicht alles zählt dabei zur formellen Freiwilligenarbeit.

1) Mitglied in einem Verein oder einer Organisation: Man kann Mitglied einer Organisation werden, indem man dieser beitritt und einen Mitgliederbeitrag bezahlt. So sind viele Schweizerinnen und Schweizer Mitglied bei Organisationen wie beispielsweise dem WWF, der REGA, der Pro Senectute, dem Roten Kreuz, Amnesty International, dem TCS oder einem Berufsverband. Das Engagement beschränkt sich letztlich auf das Bezahlen des Mitgliederbeitrages. Wie Abbildung 2.1 zeigt, sind 2019 drei Viertel der Schweizer Bevölkerung Mitglied in einem oder mehreren Vereinen oder Organisationen. Im Durchschnitt nennen diese Personen 2.7 verschiedene Bereiche (zu den Bereichen vgl. Abbildung 2.2), in welchen sie Mitglied sind.

2) Aktives Mitglied in einem Verein oder einer Organisation: Wer sich mehr engagieren will, kann aktiv an den Angeboten und Anlässen des Vereins oder der Organisation teilnehmen. Er oder sie kann im Musikverein musizieren, im Sportverein Sport treiben, im Quartierverein Kurse besuchen oder an Sitzungen einer Selbsthilfegruppe teilnehmen. 61 Prozent der Schweizer Bevölkerung machen aktiv in einem Verein oder einer Organisation mit. Diese Aktivitäten gelten noch nicht als freiwilliges Engagement.

3) Formelle Freiwilligenarbeit: Erst wer in einem Verein oder einer Organisation Aufgaben übernimmt und sich freiwillig und weitgehend unbezahlt in der Organisation engagiert, übt eine Freiwilligentätigkeit aus. Entscheidend dafür ist das eingangs erwähnte «Drittpersonen-Kriterium». Ein Engagement wird dann zu einer Freiwilligentätigkeit, wenn sie alternativ auch von einer dritten Person gegen Bezahlung ausgeführt werden könnte. Die Tätigkeit muss für die Vereinsmitglieder oder allgemein für die Gesellschaft einen Nutzen und entsprechenden Wert haben. Während im Sport-, Hobby-oder 


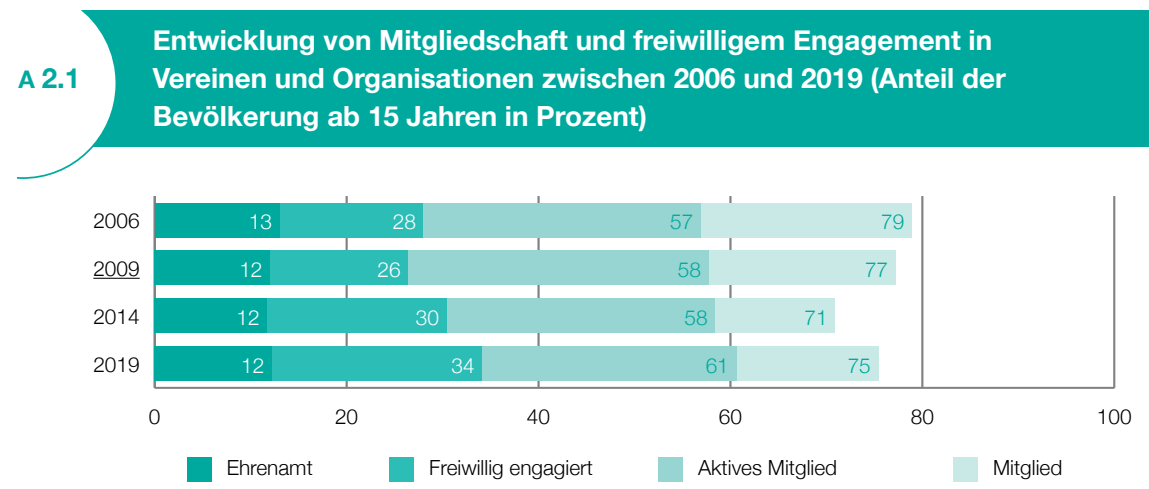

Anzahl Befragte: 2006: 7410; 2009: 6490; 2014: 5721; 2019: 5002. Lesebeispiel: Im Jahr 2009 waren 77 Prozent der Bevölkerung Mitglied in einem oder mehreren Vereinen oder Organisationen. 58 Prozent der Bevölkerung haben in Vereinen oder Organisationen aktiv mitgemacht (Aktives Mitglied), 26 Prozent haben sich freiwillig engagiert und 12 Prozent haben ein (oder mehrere) Engagement, in das sie gewählt oder berufen wurden (Ehrenamt).

Für die Vergleichbarkeit mit den Erhebungen 2006 und 2009 mussten die Werte für 2014 und 2019 analog zur damaligen Erfassung berechnet werden. Sie unterscheiden sich deshalb von den in Tabelle 1.1 angegebenen Werten. In den Erhebungen 2006 bis 2009 (CATI und CAWI) wurden die Mitgliedschaft und die aktive Tätigkeit unabhängig voneinander erfasst. In der Erhebung 2019 (CAWI und PAPI) wurden die beiden Kategorien gleichzeitig erfasst. 2019 impliziert eine «aktive Tätigkeit» die Mitgliedschaft. Für 2014 wurde der Wert in der gleichen Form neu berechnet. In den Erhebungen 2006 bis 2009 wurde die Frage nach dem freiwilligen Engagement nur Personen gestellt, die in mindestens einem Bereich aktiv tätig waren. Für den Zeitvergleich wurden die Werte für die Jahre 2014 und 2019 in der gleichen Form gebildet. In der Erhebung 2006 wurde im Falle von Engagements in mehreren Bereichen nur für das Engagement im wichtigsten bzw. zeitintensivsten Bereich erfasst, ob es sich dabei um ein gewähltes Ehrenamt handelt. Für den Zeitvergleich wurden die Werte bei den Erhebungen von 2009 bis 2019 in der gleichen Form gebildet.

Kulturverein erst die Übernahme von Aufgaben dieses Kriterium erfüllt, ist das Kriterium bei der freiwilligen Feuerwehr schon durch die aktive Mitgliedschaft erfüllt. Es kommt also auch darauf an, ob der Verein nur ein Angebot für seine Mitglieder hat oder ob er dem Gemeinwohl dient, indem er einen Nutzen und Wert für Nichtmitglieder generiert. Wer in einem Verein oder einer Organisation freiwillig übernommene Aufgaben und Arbeiten für die Mitglieder oder die Gesellschaft unentgeltlich ausführt, leistet formelle Freiwilligenarbeit. In der Schweiz üben 39 Prozent der Bevölkerung im Alter ab 15 Jahren eine formelle Freiwilligentätigkeit aus, wobei - wie wir weiter hinten sehen werden - nicht alle diese Tätigkeiten völlig unentgeltlich erbracht werden. In den ersten beiden Monitoren wurden nur Personen, die in mindestens einem Verein bzw. einer Organisation Aktivmitglied waren, zu ihrem freiwilligen Engagement befragt. Wendet man dieses Kriterium für den Zeitvergleich auch im aktuellen Monitor an, so liegt der Anteil bei 34 Prozent (vgl. Abschnitt 1.2 und Abbildung 2.1).

4) Ehrenamt in einem Verein oder einer Organisation: Wenn es sich bei der Tätigkeit in der Organisation um ein Amt handelt, in das man gewählt wird, so 
wird die Freiwilligenarbeit zum Ehrenamt. Mit der Übernahme eines Amtes wie Präsidentin, Aktuar oder Gruppenleiterin verpflichtet man sich, eine bestimmte Aufgabe für eine gewisse Zeit zu übernehmen. In der Schweiz üben 16 Prozent der Bevölkerung ein Ehrenamt aus (Erhebung 2019). Dabei kommt es relativ häufig vor, dass Personen mehr als ein Amt bekleiden. 21 Prozent der Ehrenamtlichen haben zwei Ämter, 7 Prozent sogar drei oder mehr. Die restlichen 72 Prozent üben ein Amt aus. In den vorangehenden Monitoren wurde im Falle der Freiwilligenarbeit in mehreren Bereichen nur für den wichtigsten Bereich (Erhebung 2006) bzw. die drei wichtigsten Bereiche (Erhebungen 2009 und 2014) gefragt, ob es sich dabei um ein Ehrenamt handle. Wendet man das Kriterium der ersten Erhebung auch auf die nachfolgenden Erhebungen an, so verringert sich der Anteil der Ehrenamtlichen auf 12 Prozent (vgl. Abbildung 2.1).

\section{Veränderung von Mitgliedschaft und formeller Freiwilligenarbeit über die Zeit}

Wenn man das Engagement in Vereinen und Organisationen über die Zeit analysiert, muss man die methodischen Hinweise in Abschnitt 1.2 berücksichtigen. Einerseits wurde in den Monitoren die Erhebungsmethode geändert, andererseits gab es bei der Erfassung der Kategorien leichte Unterschiede, die das Ergebnis ebenfalls mitbeeinflussen. Für den Zeitvergleich wurden die Ergebnisse für 2014 und 2019 analog zur Erfassung in den ersten beiden Monitoren berechnet. Die Werte weichen deshalb von den Werten in der Abbildung 1.1 und den publizierten Werten im Monitor 2016 (Erhebung 2014) ab.

Unter Berücksichtigung dieser methodischen Vorbehalte lassen sich aus Abbildung 2.1 die folgenden Schlüsse ziehen: Der Anteil der Mitglieder in Vereinen oder Organisationen nimmt zwischen 2006 und 2014 ab und steigt dann wieder an. Diese Veränderungen sind nur bedingt aussagekräftig; sie hängen nicht zuletzt davon ab, ob jemand seine WWF- oder TCS-Mitgliedschaft wirklich als Mitgliedschaft in einem Verein oder einer Organisation aufführt. Aussagekräftiger sind die Aktivmitgliedschaften. Hier können wir eine leichte Steigerung zwischen 2014 und 2019 feststellen, die aber auch mit dem Methodenwechsel zusammenhängen könnte. Ähnliches kann man zur Zunahme der formellen Freiwilligenarbeit sagen. Sie ist eher auf den Wechsel 
von der Telefon- auf die Online-Befragung zurückzuführen, als auf einen Boom der Freiwilligen. Bemerkenswert ist die grosse Konstanz beim Anteil an Ehrenamtlichen. In allen bisherigen Monitoren gibt etwa ein Achtel der Befragten an ein Ehrenamt auszuüben. Auch bei der erweiterten Betrachtung des Engagements in Vereinen oder Organisationen bestätigt sich das Fazit aus Abschnitt 1.2: Es ist weder ein eindeutiger Einbruch noch ein klarer Boom erkennbar. Mehr Erkenntnisse gewinnen wir erst durch eine differenziertere Betrachtung nach Bereich und Organisationstyp.

\section{Vergleich zwischen verschiedenen Vereins- und Organisationstypen}

In der Schweiz existiert eine Vielzahl an Vereinen und Organisationen, in denen man aktiv werden und sich freiwillig engagieren kann. Aus Abbildung 2.2

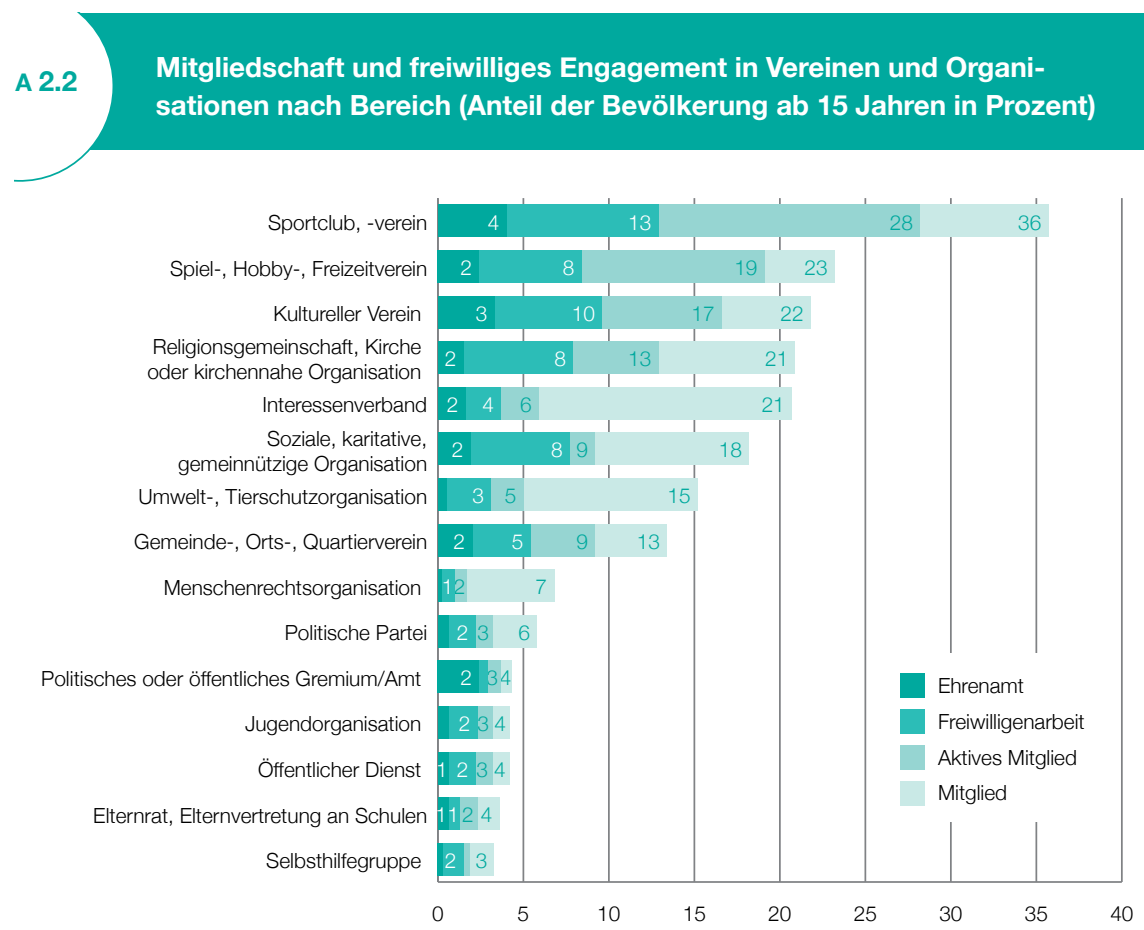

Anzahl Befragte: 4992. Lesebeispiel: 22 Prozent der Bevölkerung sind Mitglied in einem kulturellen Verein. 17 Prozent machen in einem kulturellen Verein aktiv mit (Aktives Mitglied), 10 Prozent engagieren sich freiwillig und 3 Prozent haben in einem kulturellen Verein ein Amt inne, in welches sie gewählt oder berufen wurden (Ehrenamt). 
lässt sich der Anteil an Mitgliedern, Aktivmitgliedern, formell Freiwilligen und Ehrenamtlichen für unterschiedliche Vereins- bzw. Organisationstypen herauslesen. Sowohl am meisten Mitglieder und Aktivmitglieder als auch am meisten formell Freiwillige und Ehrenamtliche zählen die Sportvereine, gefolgt von den anderen Spiel-, Hobby- und Freizeitvereinen, den kulturellen Vereinen sowie den Religionsgemeinschaften, Kirchen oder kirchennahen Organisationen. Interessenverbände sowie die verschiedenen sozialen und karitativen Organisationen, die Umwelt- und Tierschutzorganisationen und die Menschenrechtsorganisationen zeichnen sich dadurch aus, dass sie viele Mitglieder, aber nur vergleichsweise wenige Aktivmitglieder haben. Diese gemeinnützigen Vereine erbringen nicht in erster Linie Leistungen und Angebote für ihre Mitglieder, sondern für Personen ausserhalb des Vereins oder für die Umwelt. Insbesondere in den sozialen und karitativen Organisationen sind deshalb Aktivmitgliedschaften ohne freiwilliges Engagement selten.

\section{Hoher Zeitaufwand für politische und öffentliche Ämter und Gremien}

Wieviel Freiwilligenarbeit in den verschiedenen Organisationstypen genau geleistet wird, lässt sich Tabelle 2.1 entnehmen. Zunächst ist anzumerken, dass sich viele formell Freiwillige in mehr als einem Bereich engagieren. Ein gutes Viertel (27\%) nennt zwei Bereiche und ein gutes Fünftel (22\%) sogar drei und mehr Bereiche. Über 1o Prozent der Bevölkerung engagieren sich freiwillig und unentgeltlich in einem Sportverein, jeweils über 7 Prozent in einem kulturellen Verein, in einem Spiel- und Hobbyverein, in einer Religionsgemeinschaft oder Kirche oder einem sozialen und karitativen Verein. Wer sich in einem politischen oder öffentlichen Gremium engagiert, übernimmt in aller Regel auch gleich ein gewähltes Amt. In den Religionsgemeinschaften, in den Umwelt- und Tierschutzorganisationen, in den Selbsthilfegruppen sowie in den Menschenrechtsorganisationen engagiert sich dagegen die grosse Mehrheit der formell Freiwilligen, ohne ein Amt zu übernehmen.

Einen zeitlichen Aufwand von mindestens drei Stunden pro Woche leisten die Freiwilligen in den politischen und öffentlichen Gremien, in den sozialen und karitativen Organisationen, in den Jugendorganisationen sowie in den Umwelt- und Tierschutzorganisationen. Weniger als 1.5 Stunden pro 
T 2.1 Freiwilligenarbeit in Vereinen und Organisationen nach Bereich: Anteil der Bevölkerung ab 15 Jahren, Entwicklungen und Merkmale der Freiwilligen

\begin{tabular}{|c|c|c|c|c|c|c|c|}
\hline & $\begin{array}{l}\text { Freiwillige } \\
\text { (Anteil der } \\
\text { Bevölkerung } \\
\text { in \%) }\end{array}$ & $\begin{array}{l}\text { Entwicklung } \\
\text { in den letz- } \\
\text { ten Jahren }\end{array}$ & $\begin{array}{l}\text { Frauenanteil } \\
\text { (in \%) }\end{array}$ & $\begin{array}{l}\text { Mittleres } \\
\text { Alter } \\
\text { (Median) }\end{array}$ & $\begin{array}{c}\text { Mittlere } \\
\text { Anzahl } \\
\text { Jahre der } \\
\text { Tätigkeit } \\
\text { (Median) }\end{array}$ & $\begin{array}{l}\varnothing \text { zeitlicher } \\
\text { Aufwand } \\
\text { (h pro } \\
\text { Woche) }\end{array}$ & $\begin{array}{l}\text { Anteil der } \\
\text { Freiwilligen } \\
\text { mit Ehren- } \\
\text { amt (in \%) }\end{array}$ \\
\hline Sportclub, -verein & 12.9 & $\downarrow$ & 36 & 48 & 10 & 2.4 & 31 \\
\hline Kultureller Verein & 9.6 & $\uparrow$ & 50 & 52 & 6 & 2.4 & 35 \\
\hline $\begin{array}{l}\text { Spiel-, Hobby-, } \\
\text { Freizeitverein }\end{array}$ & 8.4 & $\uparrow$ & 42 & 52 & 8 & 2.1 & 29 \\
\hline $\begin{array}{l}\text { Religionsgemein- } \\
\text { schaft, Kirche, } \\
\text { kirchennahe } \\
\text { Organisation }\end{array}$ & 7.9 & $\rightarrow$ & 63 & 57 & 12 & 2.3 & 19 \\
\hline $\begin{array}{l}\text { Soziale, karitative, } \\
\text { gemeinnützige } \\
\text { Organisation }\end{array}$ & 7.7 & $\uparrow$ & 61 & 60 & 6 & 3.2 & 24 \\
\hline $\begin{array}{l}\text { Gemeinde-, Orts-, } \\
\text { Quartierverein }\end{array}$ & 5.4 & $\star \star$ & 48 & 52 & 6 & 1.7 & 37 \\
\hline Interessenverband & 3.7 & $\downarrow$ & 33 & 54 & 9 & 2.2 & 44 \\
\hline $\begin{array}{l}\text { Umwelt-, } \\
\text { Tierschutz- } \\
\text { organisation }\end{array}$ & 3.1 & $(\uparrow)$ & 56 & 56 & 6 & 3.0 & 15 \\
\hline $\begin{array}{l}\text { Politisches oder } \\
\text { Öffentliches } \\
\text { Gremium/Amt }\end{array}$ & 2.9 & $\rightarrow$ & 41 & 53 & 6 & 3.3 & 83 \\
\hline $\begin{array}{l}\text { Jugend- } \\
\text { organisation }\end{array}$ & 2.3 & $(\downarrow)$ & 50 & 29 & 6 & 3.2 & 28 \\
\hline Politische Partei & 2.2 & $\rightarrow$ & 27 & 59 & 12 & 1.4 & 28 \\
\hline Öffentlicher Dienst & 2.2 & $\downarrow$ & 29 & 46 & 10 & 2.6 & 29 \\
\hline Selbsthilfegruppe & 1.5 & ** & 54 & 58 & 5 & 1.9 & 19 \\
\hline $\begin{array}{l}\text { Elternrat, Elternver- } \\
\text { tretung an Schulen }\end{array}$ & 1.3 & $\star \star$ & 68 & 44 & 4 & 1.2 & 48 \\
\hline $\begin{array}{l}\text { Menschenrechts- } \\
\text { organisation }\end{array}$ & 1.0 & $(\uparrow)$ & 54 & 59 & 5 & 2.3 & 21 \\
\hline
\end{tabular}

Anzahl Befragte: 4992. Hinweis: * Die Einschätzung der Entwicklung in den letzten Jahren beruht auf den Angaben aus den Monitoren 2016 und 2020 (Erhebungen 2014 und 2019) sowie auf der Schweizerischen Arbeitskräfteerhebung (SAKE). $\uparrow=$ Zuwachs; $\downarrow$ = Abnahme; $\rightarrow=$ keine Veränderung; ${ }^{\star \star}$ keine Vergleichsmöglichkeit über Zeit; Zeichen in Klammern: Tendenz bzw. eingeschränkte Vergleichsmöglichkeit über Zeit. 
Woche wenden die Freiwilligen im Elternrat und in den politischen Parteien auf. Bei Letzteren dauert das Engagement dafür im Mittel bereits zwölfJahre. Über zehn Jahre sind auch die Freiwilligen in den Religionsgemeinschaften und den Kirchen, im öffentlichen Dienst und im Sport engagiert. Von kürzerer Dauer ist ein Engagement im Elternrat, aber auch in Selbsthilfegruppen oder in einer Menschenrechtsorganisation.

\section{Männer im Sportclub und öffentlichen Dienst, Frauen im Elternrat}

Frauen und Männer engagieren sich in unterschiedlichen Bereichen. In den politischen Parteien, im öffentlichen Dienst, in den Interessenverbänden und im Sport sind die Männer klar in der Mehrheit. Die Frauen engagieren sich eher im Elternrat, in Religionsgemeinschaften und der Kirche sowie in sozialen und karitativen Organisationen. Letztere zeichnen sich zusammen mit den politischen Parteien, den Selbsthilfegruppen und den Menschenrechtsorganisationen durch einen höheren Altersdurchschnitt aus. Am jüngsten sind erwartungsgemäss die Freiwilligen in Jugendorganisationen.

\section{Unterschiedliche Entwicklung in den verschiedenen Bereichen}

In Tabelle 2.1 ist auch aufgeführt, ob die formelle Freiwilligenarbeit in den verschiedenen Bereichen in den letzten Jahren eher gewachsen oder geschrumpft ist. Die Berechnung beruht einerseits auf einem Vergleich der Monitore 2016 und 2020 und andererseits auf den Zahlen der Schweizerischen Arbeitskräfteerhebung (SAKE) für den Zeitraum von 2010 bis 2016. Da insbesondere die Berechnungen der Unterschiede beim Monitor durch die Berücksichtigung von methodischen Veränderungen etwas komplexer sind, weisen wir keine Werte aus, sondern beschränken uns mittels Pfeilsymbolen auf die Aussage, ob es einen Zuwachs, eine Abnahme oder keine Veränderung gegeben hat.

Eine Abnahme des freiwilligen Engagements finden wir bei den Sportvereinen, bei den Interessenverbänden und beim öffentlichen Dienst. Tendenziell nimmt das Engagement auch bei den Jugendorganisationen ab. 
Eine Zunahme des freiwilligen Engagements lässt sich bei den kulturellen Vereinen, bei den Spiel-, Hobby- und Freizeitvereinen sowie bei den sozialen und karitativen Organisationen feststellen. Eine leichte Zunahme erkennen wir bei den Umwelt- und Tierschutzorganisationen sowie bei den Menschenrechtsorganisationen, wobei hier die Vergleichsmöglichkeiten anhand der Freiwilligen-Monitore und der SAKE eingeschränkt sind. Keine Veränderung lässt sich bei den Religionsgemeinschaften, Kirchen und kirchennahen Organisationen, bei den politischen oder öffentlichen Gremien und Ämtern sowie bei den politischen Parteien feststellen.

\subsection{Das soziale Profil der Freiwilligen in Vereinen und Organisationen}

In den bisherigen Freiwilligen-Monitoren und in verschiedenen anderen Studien konnte gezeigt werden, dass nicht alle Bevölkerungsgruppen gleich viel formelle Freiwilligenarbeit leisten (Nollert und Huser 2007; StadelmannSteffen et al. 2007, 2011; Aregger 2012; Freitag et al. 2016; Ackermann et al. 2017). Wie sehr soziodemografische und sozioökonomische Merkmale das Engagement beeinflussen, wollen wir im folgenden Abschnitt genauer betrachten.

\section{Geschlechter- und Altersunterschiede bei der formellen Freiwilligenarbeit}

In Abbildung 2.3 wird das formelle freiwillige Engagement nach Geschlecht, Alter, Sprachregion, Siedlungstyp, Nationalität und Religionszugehörigkeit dargestellt. Männer leisten etwas häufiger Freiwilligenarbeit in Vereinen oder Organisationen als Frauen. Ihr Engagement erfolgt auch öfters im Rahmen eines gewählten Ehrenamts. Vergleicht man die Altersgruppen, so ist bei den 6o-bis 74-Jährigen der Anteil der formell freiwillig engagierten Personen am höchsten. Mit Blick auf das Ehrenamt finden wir die höchste Beteiligung in der Altersgruppe der 45- bis 59-Jährigen. Sowohl bei den Frauen als bei den Männern erhöht sich der Anteil der formell freiwillig Engagierten konti- 


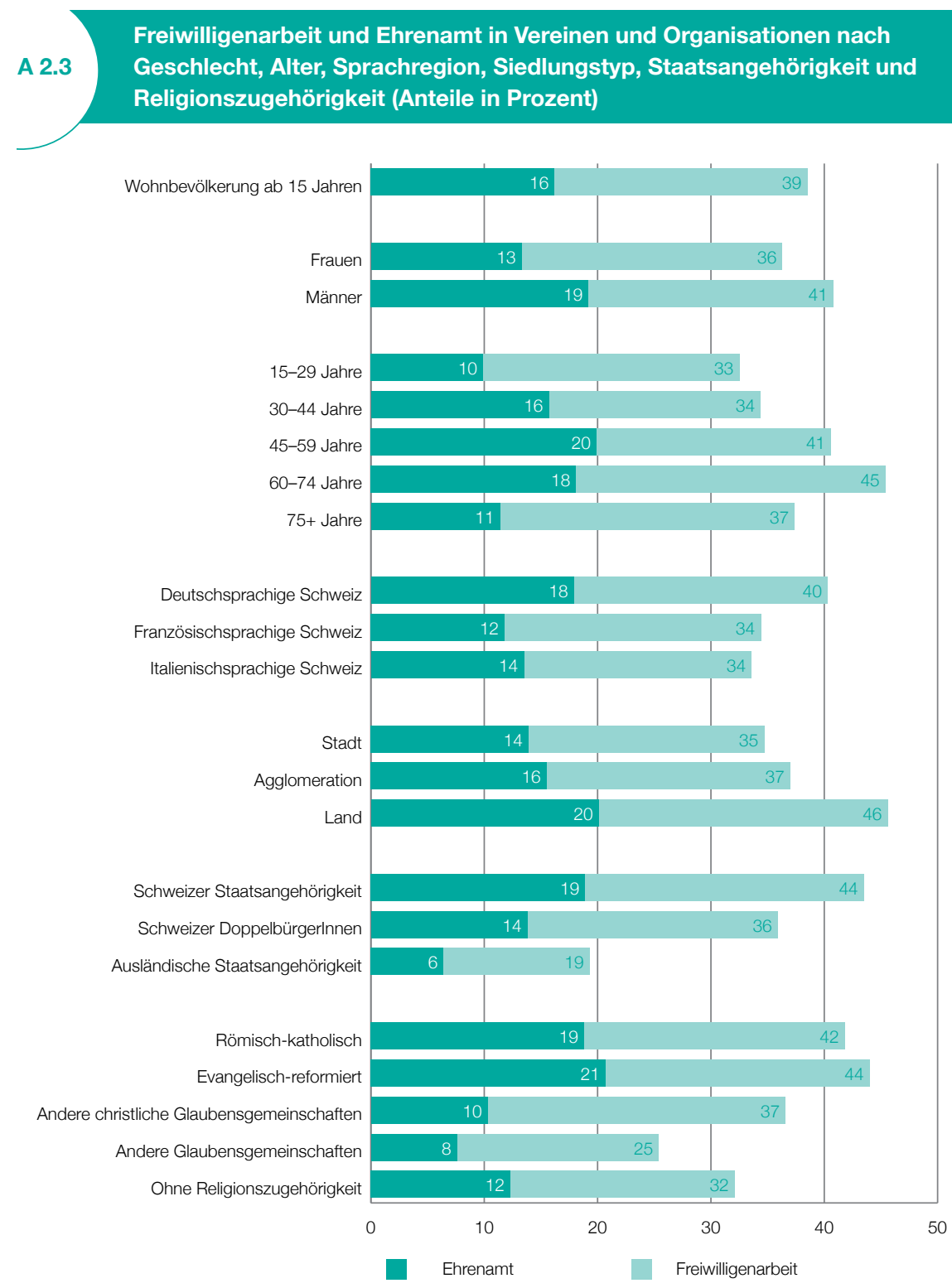

Anzahl Befragte: 5002. Lesebeispiel: 33 Prozent der 15- bis 29-Jährigen leisten Freiwilligenarbeit in Vereinen oder Organisationen, 10 Prozent leisten Freiwilligenarbeit und haben dabei ein Amt inne, in welches sie gewählt oder berufen wurden (Ehrenamt). 
nuierlich bis zur Altersgruppe der 6o- bis 74-Jährigen (Abbildung 2.4). In der Altersgruppe der 75-bis 10o-Jährigen engagieren sich Frauen sogar etwas häufiger in Vereinen oder Organisationen als Männer, wobei Letztere öfters ein Ehrenamt bekleiden.

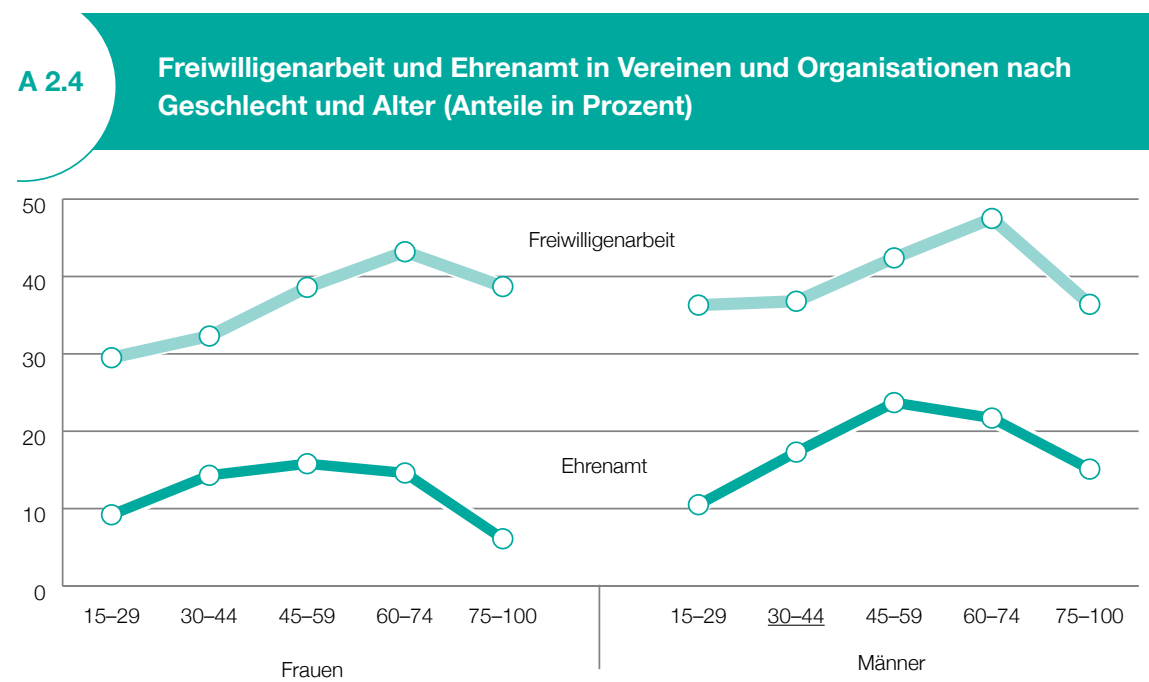

Anzahl Befragte: 5002. Lesebeispiel: 37 Prozent der 30- bis 44-jährigen Männer leisten Freiwilligenarbeit in Vereinen oder Organisationen, 17 Prozent der 30- bis 44-jährigen Männer leisten Freiwilligenarbeit und haben dabei ein Amt inne, in welches sie gewählt oder berufen wurden (Ehrenamt).

\section{Regionale Unterschiede: die Folge eines anderen Vereinsengagements}

In der Deutschschweiz ist das freiwillige und ehrenamtliche Engagement in Vereinen und Organisationen weiter verbreitet als in der französisch- und italienischsprachigen Schweiz. Ebenso ist der Anteil an formell Freiwilligen in den ländlichen Gebieten deutlich höher als in den Städten oder Agglomerationen. Diese Befunde fanden sich bereits in den früheren Monitoren (Freitag et al. 2016, 69) und in der Schweizerischen Arbeitskräfteerhebung (SAKE) (Schön-Bühlmann 2011; BFS 2015). Die sprachregionalen und StadtLand-Unterschiede zeigen sich allerdings nicht erst auf der Ebene des freiwilligen Engagements, sondern bereits auf der Ebene der Mitgliedschaft und der aktiven Teilnahme in Vereinen und Organisationen. Das heisst, 
in der Romandie und im Tessin sowie generell in städtischen Gebieten ist das formelle freiwillige Engagement vor allem auch deshalb tiefer, weil man weniger in Vereinen mitmacht.

\section{Formelles Engagement der Ausländerinnen und Ausländer}

Personen mit einem ausländischen Pass engagieren sich deutlich seltener innerhalb von Vereinen oder Organisationen als Personen mit Schweizer Pass. Für die ausländische Bevölkerung zeigt sich eine doppelte Hürde: Nicht nur sind sie seltener Aktivmitglied in einem Verein oder einer Organisation (ausländische Bevölkerung: 43 \%; Schweizerinnen und Schweizer inkl. DoppelbürgerInnen: $64 \%$ ), sie üben - wenn sie den Weg in einen Verein finden - auch anteilsmässig seltener eine freiwillige oder ehrenamtliche Tätigkeit aus. Erschwernisse oder Barrieren dürften dabei sowohl bei den Personen (z. B. Sprachkenntnisse) als auch bei den Vereinen und Organisationen zu finden sein (z. B. kulturelle Schliessung, Gewinnung von Freiwilligen, vgl. dazu Freitag et al. 2016, 186; speziell für Sportvereine Braun und Nobis 2011; Mutz und Burrmann 2015). Eine detailliertere Analyse der Migrationsbevölkerung zeigt zusätzliche Unterschiede je nach Herkunftsland und Geschlecht. So engagieren sich bei den Ausländerinnen 28 Prozent aus einem mittel oder nordeuropäischen Land in einer Organisation, aber nur 12 Prozent aus einem süd-oder osteuropäischen Land. Bei den ausländischen Männern finden sich hingegen kaum Unterschiede nach Herkunftsregion (Mittel-und Nordeuropa: $22 \%$, Süd- und Osteuropa: $19 \%$ ).

\section{Bildungs- und Einkommensunterschiede}

Personen mit einer höheren Bildung und einem höheren Haushaltseinkommen engagieren sich häufiger in Organisationen und bekleiden auch häufiger ein Amt. Wie Abbildung 2.5 zeigt, haben zudem die Erwerbsbeteiligung und der Erwerbsumfang einen Einfluss aufdas freiwillige Engagement. Teilzeiterwerbstätige, aber auch Hausfrauen bzw. -männer oder Pensionierte übernehmen etwas häufiger eine freiwillige Tätigkeit als Vollzeiterwerbstätige, Personen in Ausbildung oder Arbeitslose. Vollzeiterwerbstätige Frauen engagieren sich 

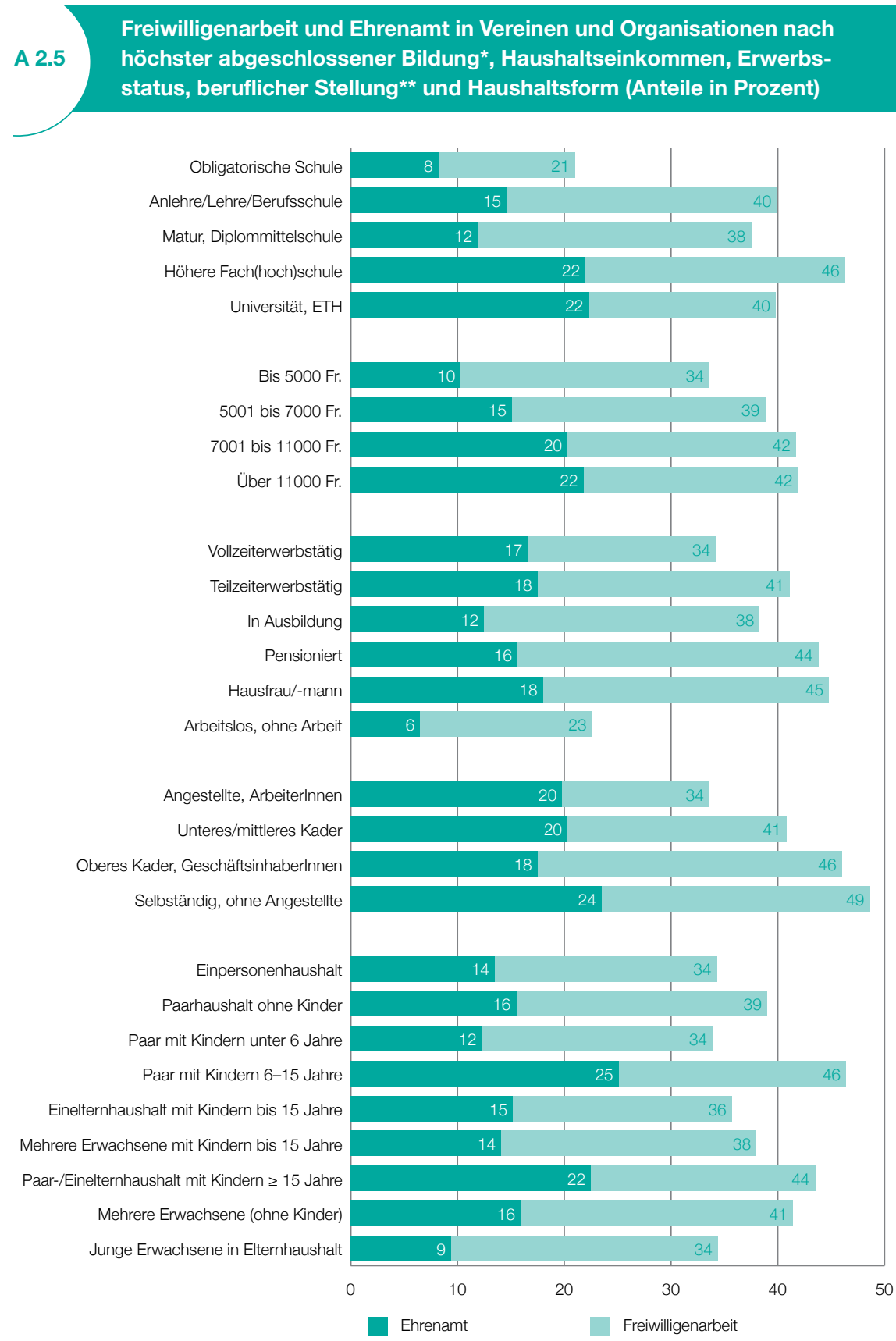

Anzahl Befragte: 5002. Hinweise: *Höchste abgeschlossene Bildung: nur Personen $\geq 30$ Jahre $(N=4150)$. ${ }^{\star \star}$ Berufliche Stellung: nur erwerbstätige Personen $(\mathrm{N}=3401)$. Lesebeispiel: 41 Prozent der teilzeiterwerbstätigen Personen leisten Freiwilligenarbeit in Vereinen oder Organisationen, 18 Prozent leisten Freiwilligenarbeit und haben dabei ein Amt inne, in welches sie gewählt oder berufen wurden (Ehrenamt). 
deutlich seltener in Vereinen und gemeinnützigen Organisationen (25\%) als vollzeiterwerbstätige Männer (39\%). Bei den Pensionierten sowie zwischen Hausfrauen und Hausmännern finden sich dagegen keine Unterschiede zwischen den Geschlechtern. Eine Erwerbstätigkeit im öffentlichen Sektor scheint das freiwillige Engagement eher zu begünstigen (42\%, Ehrenamt: $22 \%$ ) als eine Erwerbstätigkeit im privaten Sektor (36\%, Ehrenamt:20 \%). Der Blick auf die Haushaltsform zeigt, dass sich Eltern von Kindern sehr oft in Vereinen und Organisationen engagieren und Ehrenämter übernehmen. Dies gilt jedoch erst, wenn die Kinder älter als 6 Jahre sind.

\subsection{Art der Tätigkeiten der Freiwilligen}

\section{Kinder und Jugendliche als wichtige Zielgruppe}

Im vorliegenden Abschnitt wollen wir die Art der Tätigkeiten, die Zielgruppen sowie die Form und Höhe der Entschädigung etwas näher betrachten. Abbildung 2.6 gibt einerseits einen Überblick über den Personenkreis, für den man in Organisationen freiwillig und unentgeltlich tätig ist, und andererseits über den Hauptinhalt der Tätigkeit. Wie wir in Abschnitt 2.1 gesehen haben, betätigt sich rund die Hälfte der formell Freiwilligen in mehr als einer Organisation. Die Angaben in Abbildung 2.6 beziehen sich nur auf das wichtigste bzw. zeitlich aufwendigste Engagement. In knapp 4o Prozent der Fälle sind die Freiwilligen für Kinder und Jugendliche oder die gesamte Bevölkerung tätig. Ohnehin sind sie häufig für unterschiedliche Zielgruppen aktiv, was sich auch darin zeigt, dass sich die Prozentwerte auf deutlich mehr als 100 Prozent addieren. In gut einem Fünftel der Fälle engagieren sie sich für Familien oder ältere Menschen. Jeweils rund 1o Prozent der formell Freiwilligen sind für Behinderte und Pflegebedürftige, für die Umwelt und Tiere, für die Migrationsbevölkerung und für Flüchtlinge oder generell für finanziell und sozial schlechter gestellte Personen tätig. Frauen setzen sich etwas häufiger für Frauen, Ältere und Familien ein, Männer für Männer und die Bevölkerung allgemein. 

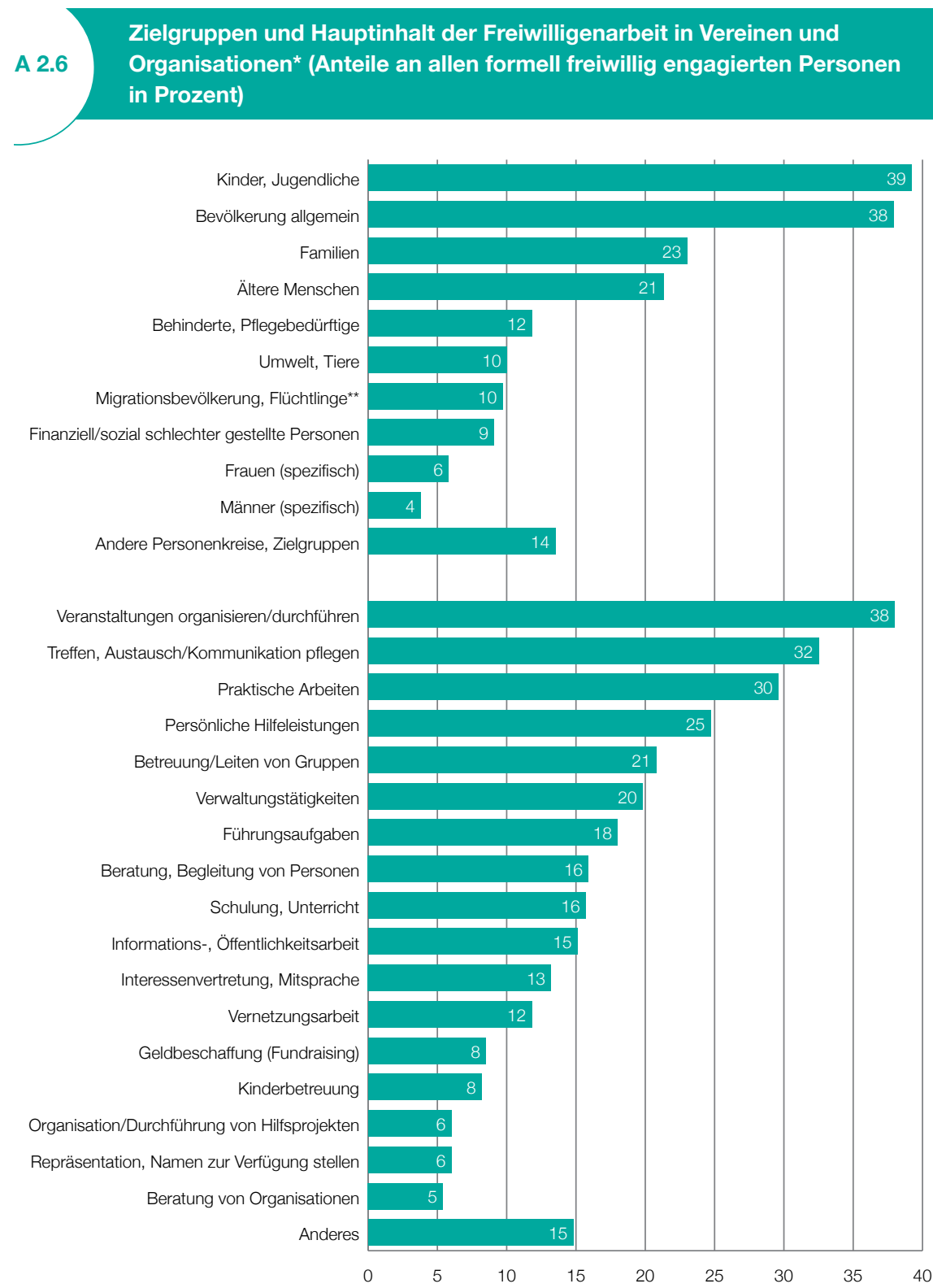

Anzahl Befragte: 1913 (Zielgruppen), 1931 (Hauptinhalt). Hinweise: * Bei einem freiwilligen Engagement in mehreren Vereinen/Organisationen bezieht sich die Angabe auf das wichtigste bzw. zeitlich aufwendigste Engagement. ${ }^{*}$ Umfasst die Kategorien «Menschen mit Migrationshintergrund, Ausländerlnnen» (7\%), «Flüchtlinge, vorläufig Aufgenommene» (5\%) und "Asylsuchende» (4\%). 


\section{Geschlechter- und Altersunterschiede bei den Tätigkeiten}

Worin der Einsatz genau besteht, lässt sich der unteren Hälfte von Abbildung 2.6 entnehmen. Wie schon in den früheren Monitoren stehen das Organisieren und Durchführen von Veranstaltungen und Anlässen an erster Stelle. Bei der Haupttätigkeit wird von über zwei Drittel der Freiwilligen mehr als ein Tätigkeitsfeld angegeben. Männer nennen häufiger Führungsaufgaben sowie Vernetzungsarbeit, Interessenvertretung, Repräsentation, Geldbeschaffung und Beratung von Organisationen. Frauen sind dafür bei den persönlichen Hilfeleistungen, bei der Kinderbetreuung und beim Beraten und Begleiten von Personen stärker vertreten (vgl. dazu auch Nadai 1996). Tätigkeiten, die von jungen Freiwilligen vergleichsweise häufig genannt werden, sind das Betreuen oder Leiten einer Gruppe, Schulung und Unterricht sowie die Kinderbetreuung. Persönliche Hilfeleistungen und die Beratung und Begleitung von Personen werden von Personen ab 6o Jahren öfters angegeben. Vernetzungsarbeit finden wir besonders häufig bei den 45- bis 59-Jährigen.

\section{Zusammenhang von freiwilliger und beruflicher Tätigkeit}

Bei 26 Prozent der formell Freiwilligen hat die freiwillige Tätigkeit etwas mit ihrer aktuellen oder früheren beruflichen Tätigkeit zu tun. Dies gilt für Frauen im gleichen Mass wie für die Männer. Es gilt aber für die älteren Freiwilligen etwas mehr als für die jüngeren und für die Ehrenamtlichen etwas mehr als für die restlichen Freiwilligen. Wie stark berufliche und freiwillige Tätigkeit miteinander korrespondieren, ist vor allem vom Bildungsstand abhängig. Personen mit hoher Bildung bringen ihre beruflichen Kenntnisse und Erfahrungen häufiger in ihre Freiwilligentätigkeit ein als Personen mit mittlerer und tiefer Bildung.

\section{Engagement ist häufig lokal ausgerichtet}

In den Tabellen und Abbildungen nicht dargestellt sind die Antworten auf die Frage, wo die formelle freiwillige Tätigkeit stattfindet. 21 Prozent 
der formell Freiwilligen geben ihre unmittelbare Nachbarschaft oder ihr Quartier an, 61 Prozent nennen ihren Wohnort. Die Mehrheit der formellen Freiwilligentätigkeiten ist also lokal ausgerichtet. Es gibt in der formellen Freiwilligenarbeiten aber durchaus auch starke Bezüge zur Region oder zur Schweiz als Ganzes. 44 Prozent der Freiwilligen nennen die Region oder den Kanton, und 27 Prozent nennen die Schweiz als Bezugsrahmen. Vergleichsweise selten wird angegeben, dass die ehrenamtliche oder freiwillige Tätigkeit nur virtuell im Internet (8\%) oder im Ausland (6\%) stattfindet.

\section{Stiftungs- und Genossenschaftsräte als Männerbastionen}

Von den Ehrenamtlichen wollte man zusätzlich wissen, was für ein Amt sie innehaben. Die Mehrheit (72 \%) der Ehrenamtlichen sind in einem Vereinsvorstand tätig. Im Weiteren werden die folgenden Funktionen bekleidet: Team-, Gruppen-, Trainingsleiterin (16\%), Milizbehörde (z. B. Schulpflege, Rechnungsprüfungskommission, $12 \%)$, Kirchenpflege, Pfarreirat (8\%), Stiftungsrat (7\%), Genossenschaftsrat (4\%) sowie anderes Amt (22\%). Bei den Team-, Gruppen- und Trainingsleitern ist das Geschlechterverhältnis ausgeglichen. In der Kirchenpflege und den Pfarreiräten sowie bei den «anderen Ämtern» sind die Frauen ganz leicht in der Mehrzahl. Bei allen anderen Tätigkeiten herrscht ein kleinerer oder grösserer Männerüberschuss. Am grössten ist er bei den Stiftungs- und Genossenschaftsräten, wo auf drei Männer eine Frau kommt, sowie in den Milizämtern, wo es anderthalbmal mehr Männer hat.

\section{Verschiedene Formen der Wertschätzung und Entschädigung}

Freiwilligenarbeit ist per definitionem nicht nur freiwillig, sondern auch unentgeltlich. Kleine Entschädigungen oder andere Formen von Vergütungen und Belohnungen sind aber durchaus üblich, ohne dass die Freiwilligenarbeit damit zur Erwerbsarbeit würde. Eine wichtige Form der Anerkennung für die geleistete Arbeit sind Jahresessen oder vergleichbare Erkenntlichkeiten (vgl. Abbildung 2.7). Einem Drittel der formell Freiwilligen wird auf diese Weise für ihren Einsatz gedankt. Eine Spesenentschädigung, das heisst eine 


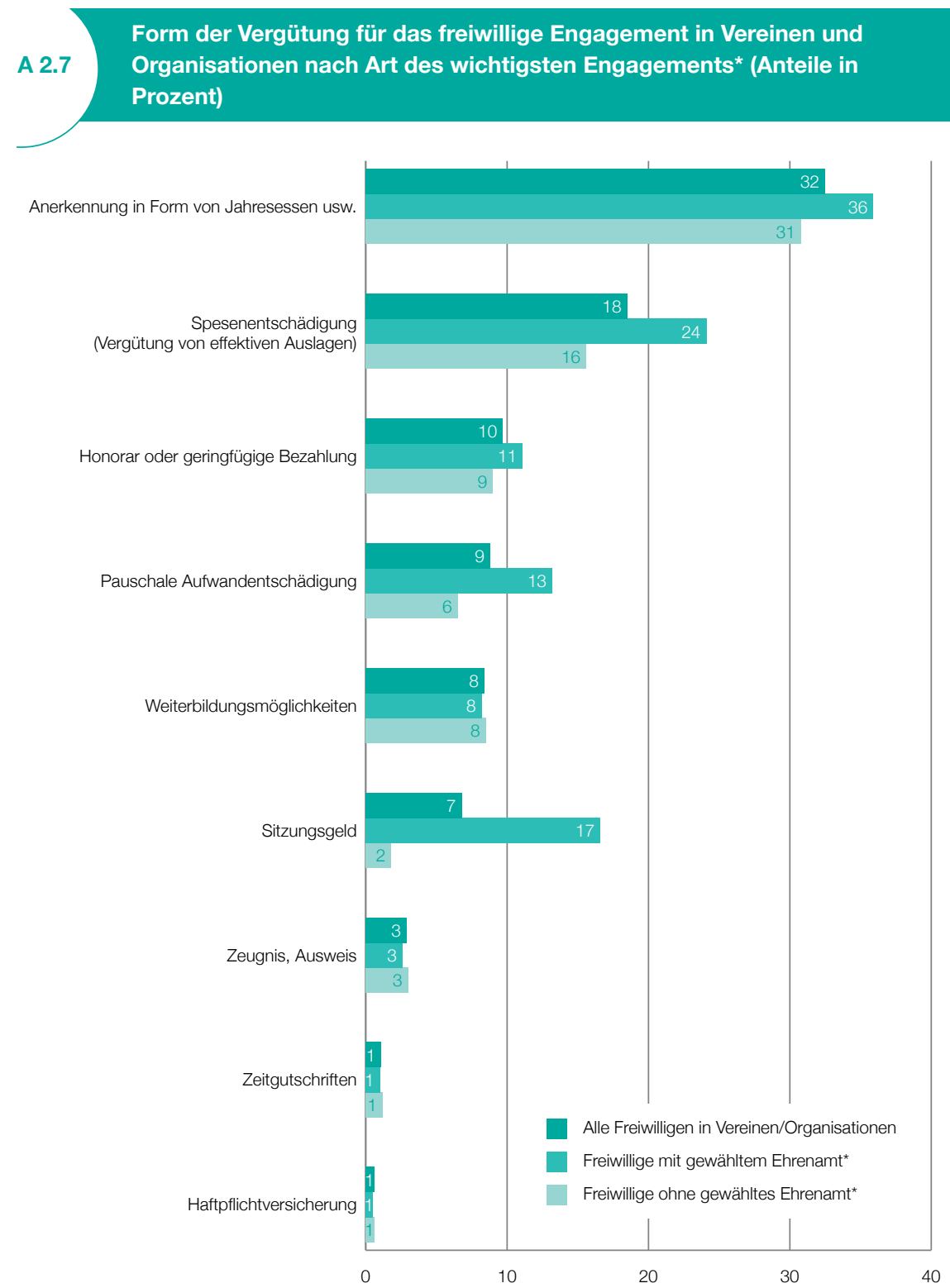

Anzahl Befragte: 1923. Hinweis: * Bei einem freiwilligen Engagement in mehreren Vereinen/Organisationen bezieht sich die Angabe auf das wichtigste bzw. zeitlich aufwendigste Engagement. 
Vergütung für die effektiven Auslagen, erhält ein Fünftel der formell Freiwilligen. Weitergehende finanzielle Vergütungen erfolgen als Honorar oder geringfügige Bezahlung (10\%), als pauschale Aufwandentschädigung (9\%) oder als Sitzungsgeld (7\%). Mit Letzterem werden Ehrenamtliche deutlich häufiger entschädigt als die restlichen formell Freiwilligen. Die Möglichkeit, Weiterbildungen zu besuchen, können 8 Prozent der formell Freiwilligen nutzen. Nur selten erhalten sie ein Zeugnis (3\%), eine Zeitgutschrift (1\%) oder eine Haftpflichtversicherung (1\%).

\section{Ein Fünftel der formell Freiwilligen wird finanziell entschädigt}

Für die weiteren Analysen in Tabelle 2.2 und Abbildung 2.8 werden einerseits die monetären Vergütungen (Honorar, pauschale Aufwandentschädigung und Sitzungsgeld) und andererseits die Spesenentschädigung und alle nichtmonetären Vergütungen (Jahresessen, Weiterbildungsmöglichkeiten, Zeugnis, Zeitgutschrift, Haftpflichtversicherung) zusammengefasst: 44 Prozent der formell Freiwilligen bekommen keine Vergütungen oder Entschädigungen. 35 Prozent erhalten Spesenentschädigungen oder nichtmonetäre Vergütungen, während 21 Prozent finanziell vergütet werden. Im Schnitt betragen die finanziellen Vergütungen 152 Franken pro Monat und Person. Für diese Vergütung arbeiten die Freiwilligen durchschnittlich 14.9 Stunden pro Monat. Eine Stunde wird für diese Gruppe also mit rund 1o Franken vergütet. Die Durchschnittswerte sind hier aber nur bedingt aussagekräftig: Gut 4o Prozent erhalten auf eine Stunde nicht mehr als 5 Franken, etwa 20 Prozent erhalten über 20 Franken, 4 Prozent über 6o Franken. Gegenüber 2014 können wir keinen Anstieg der finanziellen Entschädigungen erkennen. Zukünftig wird aber zu klären sein, inwiefern diese finanziell entschädigten Tätigkeiten überhaupt noch als Freiwilligenarbeit gelten können oder ob das teilweise vergütete gemeinnützige Engagement separat zu erfassen ist. 


\begin{tabular}{|c|c|c|c|c|c|}
\hline & $\begin{array}{l}\text { Finanzielle } \\
\text { Vergütung über } \\
\text { Spesenentschä- } \\
\text { digung hinaus } \\
\text { (in \%) }\end{array}$ & $\begin{array}{l}\text { Spesenentschä- } \\
\text { digung oder } \\
\text { andere } \\
\text { nicht-finanzielle } \\
\text { Vergütung } \\
\text { (in \%) }\end{array}$ & $\begin{array}{l}\text { Keine } \\
\text { Vergütung } \\
\text { (in \%) }\end{array}$ & $\begin{array}{l}\text { Höhe der finanzi- } \\
\text { ellen Vergütung }{ }^{\star \star} \\
\text { (Durchschnitt pro } \\
\text { Monat) }\end{array}$ & $\begin{array}{l}\text { Zeitlicher Aufwand } \\
\text { für finanziell } \\
\text { vergütetes } \\
\text { Engagement } \\
\text { (Durchschnitt pro } \\
\text { Monat) }\end{array}$ \\
\hline Alle Freiwilligen & 21 & 35 & 44 & 152 & 14.9 \\
\hline \multicolumn{6}{|l|}{ Geschlecht } \\
\hline Männer & 23 & 33 & 44 & 175 & 14.5 \\
\hline Frauen & 20 & 37 & 43 & 123 & 15.4 \\
\hline \multicolumn{6}{|l|}{ Alter } \\
\hline 15-44 Jahre & 21 & 35 & 44 & 102 & 14.3 \\
\hline 45-64 Jahre & 23 & 33 & 44 & 187 & 14.0 \\
\hline 65-100 Jahre & 18 & 39 & 43 & (167) & (18.2) \\
\hline \multicolumn{6}{|c|}{ Art des wichtigsten Engagements } \\
\hline Ehrenamt* & 32 & 35 & 33 & 200 & 15.1 \\
\hline Kein Ehrenamt* ${ }^{\star}$ & 16 & 35 & 49 & 99 & 14.7 \\
\hline
\end{tabular}

Anzahl Befragte: 1923. Hinweise: * Bei einem freiwilligen Engagement in mehreren Vereinen/Organisationen bezieht sich die Angabe auf das wichtigste bzw. zeitlich aufwendigste Engagement. ${ }^{\star *}$ Umfasst die Kategorien "pauschale Aufwandentschädigung", "Honorar oder geringfügige Bezahlung" und "Sitzungsgeld». *** Umfasst die Kategorien "Spesenentschädigung", "Anerkennung in Form von Jahresessen etc.", "Weiterbildungsmöglichkeiten", "Zeugnis, Ausweis", "Zeitgutschriften" und

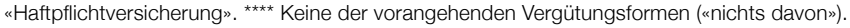

\section{Höhere finanzielle Entschädigungen für Männer und ältere Personen}

Männer und Frauen erhalten gleich häufig Vergütungen. Männer werden aber etwas häufiger monetär entschädigt, Frauen eher nichtmonetär. Die finanziellen Entschädigungen sind zudem für die Männer klar höher als für die Frauen, obwohl Letztere sogar etwas mehr für die finanziellen Entschädigungen arbeiten. Der Anteil an Personen, die finanziell entschädigt werden, nimmt mit dem Alter kaum zu; die finanziellen Vergütungen werden aber mit dem Alter höher. Die geschlechtsspezifischen Unterschiede bei den finanziellen Entschädigungen liegen auch daran, dass Männer häufiger als Frauen ein Amt inne haben und ein gewähltes Amt eher und deutlich höher entschädigt wird als die restliche formelle Freiwilligenarbeit. 


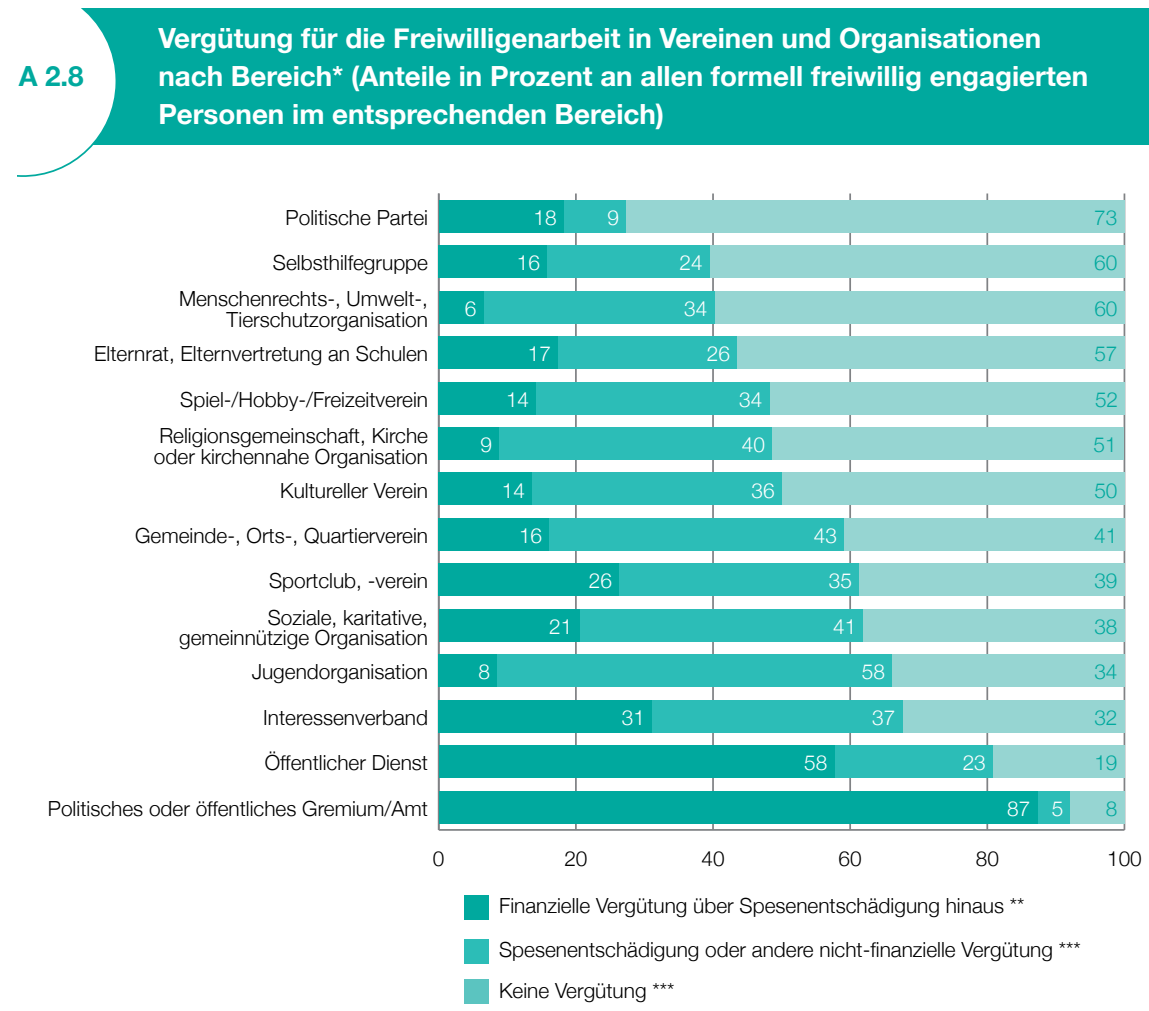

Anzahl Befragte: 1923. Hinweise: * Bei einem freiwilligen Engagement in mehreren Vereinen/Organisationen bezieht sich die Angabe auf das wichtigste bzw. zeitlich aufwendigste Engagement. ${ }^{\star \star}$ Umfasst die Kategorien «pauschale Aufwandentschädi-

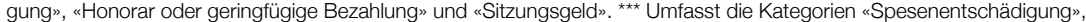
"Anerkennung in Form von Jahresessen usw.», «Weiterbildungsmöglichkeiten», «Zeugnis, Ausweis», "Zeitgutschriften» und «Haftpflichtversicherung». ${ }^{\star \star \star \star ~ K e i n e ~ d e r ~ v o r a n g e h e n d e n ~ V e r g u ̈ t u n g s f o r m e n ~(« n i c h t s ~ d a v o n ») . ~}$

\section{Politische Ämter werden meistens entschädigt}

Die deutlichsten Unterschiede bei den Vergütungen zeigen sich aber nicht zwischen Geschlecht und Alter, sondern zwischen den Vereins- und Organisationstypen (vgl. Abbildung 2.8). Freiwilligenarbeit in politischen und öffentlichen Gremien, die meist mit einem Amt einhergeht, ist in 87 Prozent der Fälle finanziell entschädigt. Im öffentlichen Dienst erhalten 58 Prozent der Freiwilligen finanzielle Vergütungen und in Interessenverbänden und Sportvereinen sind es über ein Viertel (vgl. zu den Sportvereinen auch Lamprecht et al. 2012). Ganz anders sieht es bei den Menschenrechts-, Umwelt- und Tierschutzorganisationen, bei den Religionsgemeinschaften, Kirchen und kirchennahen Organisationen sowie bei den Jugendorganisationen aus, wo 
weniger als 1o Prozent über allfällige Spesenentschädigungen hinaus finanzielle Vergütungen erhalten. Bei den Jugendorganisationen erhält die Mehrheit der Freiwilligen dafür eine nichtmonetäre Entschädigung. 


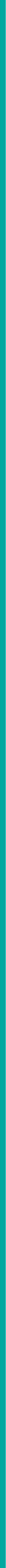


1 Freiwilligkeit

${ }_{2}$ Formelle Freiwilligenarbeit und Ehrenamt

3 Informelle Freiwilligenarbeit

4 Spenden und weitere Formen von Freiwilligkeit

5 Motive, Mobilisierung und Potenzial

6

Politisches Engagement, Vertrauen und die Freiwilligkeit

Untersuchungsmethode und Stichprobe

Literaturverzeichnis 



\subsection{Informelle Freiwilligentätigkeiten im eigentlichen und weiten Sinne}

\section{Abgrenzung der informellen Freiwilligenarbeit zur Haus- und Familienarbeit}

Das freiwillige und unentgeltliche Engagement ausserhalb von Vereinen und Organisationen wird als informelle Freiwilligenarbeit bezeichnet. Beispiele für informelle Freiwilligenarbeit sind Kinder hüten, Personen betreuen oder pflegen, Transporthilfe oder Mithilfe bei Anlässen und Festlichkeiten. Die Herausforderung bei der Erfassung von informeller Freiwilligentätigkeit besteht in der Abgrenzung zur Haus- und Familienarbeit. Im FreiwilligenMonitor wie in der Schweizerischen Arbeitskräfteerhebung (SAKE) gehören die verschiedenen Pflege- und Betreuungsaufgaben dann zur Freiwilligenarbeit, wenn sie Personen ausserhalb des eigenen Haushalts zugute kommen (vgl. dazu Abschnitt 1.2). Diese Grenzziehung ist für die Betreuenden oft nicht ganz nachvollziehbar und trägt den veränderten Wohn- und Familienformen nur bedingt Rechnung. Vor diesem Hintergrund unterscheiden wir im Monitor 2020 zusätzlich, ob zwischen den Freiwilligen und den Betreuten eine Verwandtschaftsbeziehung besteht. Wer informelle Freiwilligenarbeit im eigentlichen Sinne leistet, kümmert sich um Personen, mit denen er oder sie nicht verwandt ist. Informelle Freiwilligenarbeit im weiten Sinne umfasst zusätzlich zur Freiwilligenarbeit im eigentlichen Sinne auch die Freiwilligen, die sich um verwandte Personen kümmern, die nicht im gleichen Haushalt leben.

\section{Das informelle Freiwilligenengagement bleibt unverändert hoch}

Gemäss der neuesten Monitor-Erhebung haben in den letzten vier Wochen vor der Befragung 35 Prozent der Schweizer Bevölkerung ab 15 Jahren informelle Freiwilligenarbeit im eigentlichen Sinne und 46 Prozent informelle Freiwilligenarbeit im weiten Sinne geleistet. Der Anteil der Bevölkerung, der informelle Freiwilligenarbeit im eigentlichen Sinne leistet, lässt sich auch im 
Freiwilligen-Monitor 2016 berechnen. Bereits im damaligen Erhebungsjahr 2014 haben 35 Prozent der Bevölkerung informelle Freiwilligenarbeit im eigentlichen Sinne geleistet. In der SAKE von 2016 liegt der Wert für informelle Freiwilligenarbeit mit 32 Prozent etwas tiefer, was auch dadurch erklärt werden kann, dass in der SAKE die punktuelle Mithilfe bei Veranstaltungen, Anlässen und Projekten nicht erhoben wird.

\section{Care-Arbeit und informelle Freiwilligenarbeit}

Abbildung 3.1 gibt eine Übersicht über die verschiedenen Formen des informellen Freiwilligenengagements. Dabei wird unterschieden zwischen Pflege- und Betreuungsaufgaben, die man auch als unbezahlte Care-Arbeit zusammenfassen könnte, und anderen Formen des informellen Engagements. Bei der unbezahlten Care-Arbeit steht die Betreuung von Kindern vor der Betreuung und Pflege von älteren Personen an erster Stelle. 19 Prozent der Bevölkerung leisten informelle Freiwilligenarbeit, indem sie Kinder betreuen. 8 Prozent betreuen dabei auch Kinder, mit denen sie nicht verwandt sind,

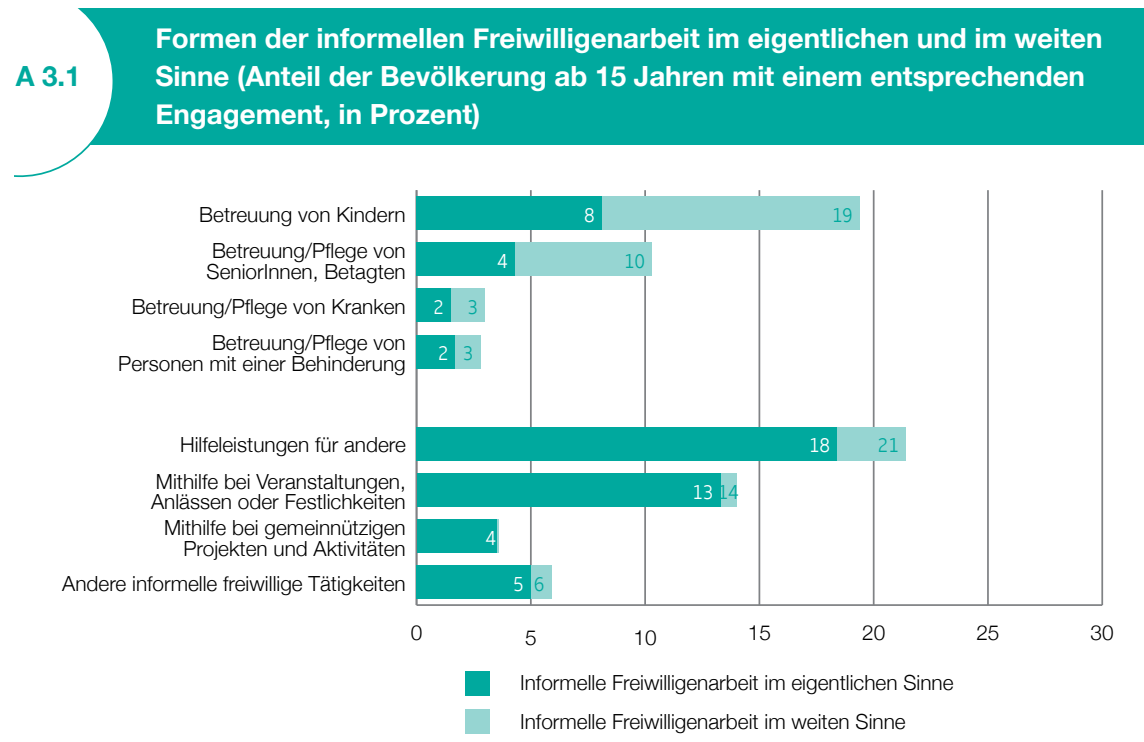

Anzahl Befragte: 4971. Lesebeispiel: 3 Prozent der Bevölkerung engagieren sich informell freiwillig in Form der Betreuung und Pflege von kranken Personen (informelle Freiwilligenarbeit im weiten Sinne). Bei 2 Prozent der Bevölkerung profitieren dabei auch nichtverwandte Personen (Freiwilligenarbeit im eigentlichen Sinne). 
während 11 Prozent sich ausschliesslich um verwandte Kinder kümmern. 10 Prozent der Bevölkerung leisten unbezahlte Care-Arbeit für betagte Personen. Dazu gehört auch die Pflege und Betreuung der eigenen Eltern, sofern diese nicht im eigenen Haushalt wohnen. 4 Prozent der Bevölkerung leisten unbezahlte Care-Arbeit auch für betagte Personen, mit denen sie nicht verwandt sind. Fasst man alle in Abbildung 3.1 geleisteten Betreuungs- und Pflegearbeiten zusammen, so leisten 28 Prozent der Bevölkerung freiwillige und unentgeltliche Care-Arbeit für Personen ausserhalb des eigenen Haushalts. 12 Prozent leisten freiwillige Care-Arbeit im eigentlichen Sinne, indem sie auch nichtverwandte Personen pflegen und betreuen.

\section{Andere Hilfeleistungen und Mithilfe bei Veranstaltungen und Projekten}

Bei den anderen Formen des informellen Engagements stehen die Hilfeleistungen für Andere an erster Stelle vor der Mithilfe an Anlässen und gemeinnützigen Projekten. Fasst man diese Formen des informellen freiwilligen Engagements analog zur Care-Arbeit zusammen, so üben 33 Prozent eine entsprechende informelle Freiwilligentätigkeit aus. Da von diesen Hilfeleistungen nicht in erster Linie Verwandte profitieren, ist hier der Anteil an informeller Freiwilligenarbeit im eigentlichen Sinne deutlich höher als bei der Care-Arbeit. 30 Prozent der Bevölkerung üben eine andere informelle Freiwilligentätigkeit aus, die sich auch an nichtverwandte Personen richtet.

\section{Hilfeleistungen für Verwandte, Bekannte und andere Personen}

Einen genaueren Einblick in den Personenkreis, der vom informellen Engagement profitiert, gibt Tabelle 3.1. Hier ist genauer ausgeführt, in welcher Beziehung man zu den Personen steht, für die man informelle Freiwilligenarbeit leistet, und wie die Unterscheidung zwischen informeller Freiwilligenarbeit im eigentlichen und weiten Sinne gebildet wird. Bei der Betreuung und Pflege von Kranken sieht dies wie folgt aus: 50 Prozent kümmern sich um nächste Verwandte, 18 Prozent um andere Verwandte und wiederum 50 Prozent um Personen, mit denen sie nicht verwandt sind. Da die gleiche Person verwandte 
und nichtverwandte Kranke pflegen kann, addieren sich die Prozentwerte nicht auf 100 Prozent. Bei der Mithilfe bei Veranstaltungen, Anlässen, und Festlichkeiten ist man hauptsächlich mit und für Personen tätig, die man persönlich kennt. Personen, die man nicht persönlich kennt, profitieren am ehesten im Rahmen von gemeinnützigen Projekten von der Mithilfe anderer.

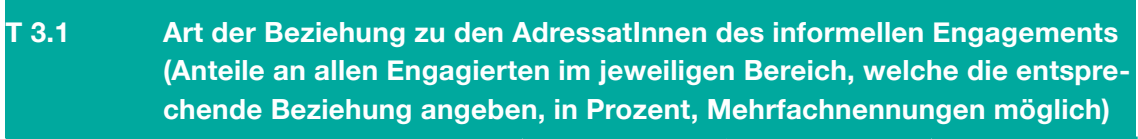

\section{Betreuung/Pflege von Personen}

\begin{tabular}{lccc} 
& $\begin{array}{c}\text { Nächste } \\
\text { Verwandte }\end{array}$ & $\begin{array}{c}\text { Andere } \\
\text { Verwandte }\end{array}$ & $\begin{array}{c}\text { Andere } \\
\text { Personen }\end{array}$ \\
\hline Betreuung von Kindern & 60 & 12 & 42 \\
Betreuung/Pflege von Seniorlnnen, Betagten & 57 & 14 & 42 \\
Betreuung/Pflege von Kranken & 50 & 18 & 50 \\
Betreuung/Pflege von Personen mit einer Behinderung & 33 & 11 & 59
\end{tabular}

Andere Formen des informellen Engagements

\begin{tabular}{|c|c|c|c|}
\hline & $\begin{array}{l}\text { Verwandte } \\
\text { Personen }\end{array}$ & $\begin{array}{l}\text { Persönlich } \\
\text { Bekannte }\end{array}$ & $\begin{array}{l}\text { Nicht persönlich } \\
\text { Bekannte }\end{array}$ \\
\hline Hilfeleistungen für andere & 50 & 82 & 12 \\
\hline $\begin{array}{l}\text { Mithilfe bei Veranstaltungen, Anlässen } \\
\text { oder Festlichkeiten }\end{array}$ & 26 & 84 & 22 \\
\hline Mithilfe bei gemeinnützigen Projekten & 18 & 68 & 47 \\
\hline Andere informelle freiwillige Tätigkeiten & 40 & 69 & 23 \\
\hline
\end{tabular}

Anzahl Befragte: 2228. Lesebeispiel: Bei 60 Prozent der Personen, die informell freiwillig Kinder betreuen, handelt es sich um nächste Verwandte, bei 12 Prozent um andere Verwandte und bei 42 Prozent um nichtverwandte Personen. Da unterschiedliche Kinder betreut werden können, addieren sich die Werte nicht auf 100 Prozent. Hinweis: * Im Fragebogen werden Kinder, Eltern und Grosskinder als «nächste Verwandte» angegeben.

\section{Pensionierte leisten viel unbezahlte Care-Arbeit}

Detaillierte Informationen zu den Personen, welche die verschiedenen Arten des informellen Engagements leisten, gibt Tabelle 3.2. Der Teil des informellen Engagements, die unter Care-Arbeit fällt, wird mehrheitlich von Frauen ausgeübt. Bei den anderen Hilfeleistungen ist das Geschlechterverhältnis ausgeglichener, zudem ist das Durchschnittsalter tiefer als bei 
der Care-Arbeit. Besonders zeitintensiv ist die Betreuung von Kindern, für die durchschnittlich 4.6 Stunden pro Woche aufgewendet wird. Über drei Stunden informelles Engagement pro Woche fliesst zudem in die Betreuung und Pflege von Betagten und Kranken. Eine genauere - hier nicht dargestellte - Analyse der einzelnen Betreuungsaufgaben zeigt, dass die Betreuung von Kindern insbesondere von Frauen und in etwas geringerem Masse von Männern im Alter von jeweils zo bis 44 Jahren und noch verstärkt im Alter von 60 bis 74 Jahren geleistet wird. Das heisst, das vielzitierte Enkelhüten ist nicht einfach ein Klischee, sondern macht wirklich einen beachtlichen Teil des informellen Engagements aus.

\begin{tabular}{|c|c|c|c|c|c|}
\hline & \multicolumn{3}{|c|}{$\begin{array}{l}\text { Informelle Freiwilligenarbeit } \\
\text { im weiten Sinne }\end{array}$} & \multicolumn{2}{|c|}{$\begin{array}{l}\text { Informelle Freiwilligenarbeit im } \\
\text { eigentlichen Sinne }\end{array}$} \\
\hline & $\begin{array}{l}\text { Frauenanteil } \\
\text { (in Prozent) }\end{array}$ & $\begin{array}{l}\text { Mittleres Alter } \\
\text { (Median) }\end{array}$ & $\begin{array}{l}\text { Ø zeitlicher } \\
\text { Aufwand } \\
\text { (h pro Woche) }\end{array}$ & $\begin{array}{l}\text { Frauenanteil } \\
\text { (in Prozent) }\end{array}$ & $\begin{array}{l}\text { Mittleres Alter } \\
\text { (Median) }\end{array}$ \\
\hline Betreuung von Kindern & 68 & 51 & 4.6 & 74 & 41 \\
\hline $\begin{array}{l}\text { Betreuung/Pflege von } \\
\text { Seniorlnnen, Betagten }\end{array}$ & 66 & 57 & 3.3 & 62 & 62 \\
\hline Betreuung/Pflege von Kranken & 64 & 56 & 3.1 & 67 & 60 \\
\hline $\begin{array}{l}\text { Betreuung/Pflege von Personen } \\
\text { mit einer Behinderung }\end{array}$ & 64 & 57 & 2.9 & 63 & 63 \\
\hline Hilfeleistungen für andere & 55 & 48 & 1.5 & 54 & 47 \\
\hline $\begin{array}{l}\text { Mithilfe bei Veranstaltungen, } \\
\text { Anlässen oder Festlichkeiten }\end{array}$ & 54 & 46 & 1.7 & 54 & 46 \\
\hline $\begin{array}{l}\text { Mithilfe bei gemeinnützigen } \\
\text { Projekten und Aktivitäten }\end{array}$ & 42 & 47 & 2.2 & 41 & 47 \\
\hline $\begin{array}{l}\text { Andere informelle freiwillige } \\
\text { Tätigkeiten }\end{array}$ & 56 & 53 & 2.6 & 55 & 52 \\
\hline
\end{tabular}

Anzahl Befragte: 2294.

Die Betreuung und Pflege von betagten Personen wird hauptsächlich von Frauen im Alter von 45 bis 74 Jahren sowie in etwas geringerem Masse von Männern im Alter von 6o bis 74 Jahren geleistet. In diesem Fall werden wohl häufig die hochbetagten Eltern oder Schwiegereltern betreut und gepflegt. Bemerkenswert dabei ist, dass die Kinder, welche diese Betreuungsarbeit für 
ihre Eltern leisten, häufig schon selber im Pensionsalter sind. Vor dem Pensionsalter sind es mehrheitlich Frauen, welche diese Arbeit übernehmen. Bei den Formen des informellen Engagements, die nicht zur Care-Arbeit gehören, gibt es weder grosse Geschlechter- noch Altersunterschiede. Sie wird von Männern und Frauen in allen Altersphasen in ähnlichem Ausmass geleistet.

\section{Nachbarschaftshilfe ist weit verbreitet}

Die Probleme bei der Erfassung der informellen Freiwilligenarbeit sind einerseits die dargestellte Abgrenzung zur Haus- und Familienarbeit, andererseits die Frage, wie gross und dauerhaft eine Hilfeleistung sein muss, damit sie als informelle Freiwilligenarbeit gilt. Das «Drittpersonen-Kriterium» sagt nur, dass die erbrachte Hilfeleistung auch gegen Bezahlung an eine Drittperson übertragen werden könnte. Dies würde aber bereits das Leeren des Briefkastens bei einer kurzen Ferienabwesenheit einschliessen. Gegen ein Entgelt würde die Post die Sendungen zurückhalten und erst nach den Ferien liefern. Aufgrund des zeitlichen Aufwands, den die Befragten für ihr informelles Freiwilligenengagement angeben (vgl. Tabelle 3.2), können wir annehmen, dass solche kleinen nachbarschaftlichen Hilfeleistungen von ihnen nicht zur Freiwilligenarbeit gezählt werden.

Würden die Befragten die sporadische Nachbarschaftshilfe auch als Freiwilligenarbeit betrachten, würde sich die Zahl der Freiwilligen deutlich erhöhen. 72 Prozent der Befragten geben nämlich an, in den letzten 12 Monaten kleine nachbarschaftliche Hilfeleistungen erbracht zu haben - wie zum Beispiel Aushelfen mit Kleinigkeiten, Briefkasten leeren, Pakete in Empfang nehmen, Pflanzen giessen oder Haustiere füttern. Der Unterschied zur oben erfassten informellen Freiwilligenarbeit besteht einerseits darin, dass es sich nur um kleine Hilfestellungen handelt. Andererseits wurden als zeitlicher Bezugsrahmen nicht die letzten vier Wochen, sondern die letzten zwölf Monate angegeben. Das Beispiel verdeutlicht aber, wie sehr die Angaben zum Ausmass der informellen Freiwilligenarbeit davon abhängen, wie man informelle Freiwilligkeit definiert und erfasst.

Bei der Erbringung von kleinen nachbarschaftlichen Hilfeleistungen gibt es nur einen bescheidenen Geschlechterunterschied (Frauen: 74 \%; Männer $69 \%$ ), und auch ein Altersunterschied ist kaum erkennbar (ab zo Jahren betei- 
ligen sich in allen Altersgruppen mindestens 70 \% an der Nachbarschaftshilfe). Besonders bemerkenswert ist aber, dass es keinen nennenswerten Stadt-LandUnterschied gibt. Die Bereitschaft, Nachbarschaftshilfe zu leisten, ist in der Stadt (70 \%), in der Agglomeration (72\%) und auf dem Land (72\%) praktisch gleich hoch. In der Deutschschweiz (73\%) ist sie allerdings leicht höher als in der Romandie (69\%) und in der italienischsprachigen Schweiz (66\%).

\subsection{Das soziale Profil der informell Freiwilligen}

Wie bei der formellen Freiwilligenarbeit steht auch die Bereitschaft, informelle Freiwilligenarbeit zu leisten, in Zusammenhang mit der sozialen Lage einer Person. Abbildung 3.2 macht die Unterschiede nach Geschlecht, Alter, Sprachregion, Siedlungstyp, Staatsangehörigkeit und Religionszugehörigkeit sichtbar. Auf den Einfluss von Geschlecht und Alter sind wir schon in Abschnitt 3.1 eingegangen: Frauen und Personen im frühen Pensionsalter leisten am meisten informelle Freiwilligenarbeit. Etwas detailliertere Informationen dazu lassen sich Abbildung 3.3 entnehmen. Dass sowohl Frauen und Männer im Alter von 6o bis 74 Jahre am meisten informelle Freiwilligenarbeit im weiten Sinne ausüben, die vor allem verwandten Personen zu Gute kommt, liegt daran, dass sich Pensionierte sowohl häufig um ihre Enkel als auch um ihre betagten Eltern oder Schwiegereltern kümmern.

\section{Geschlechter-, Alters- und Nationalitätenunterschiede bei der informellen Freiwilligenarbeit}

Nicht nur die Geschlechter- und Altersunterschiede, sondern auch die meisten anderen in Abbildung 3.2 dargestellten Unterschiede nach Sprachregion, Siedlungstyp, Nationalität und Religionszugehörigkeit kennt man bereits aus den früheren Monitoren und aus der SAKE (Bühlmann und Freitag 2007; Nollert und Huser 2007; Stadelmann-Steffen et al. 2010; BFS 2015; Freitag et al. 2016). In der Deutschschweiz wird häufiger informelle Freiwilligenarbeit geleistet als in der französisch-und italienischsprachigen Schweiz. Mehr 

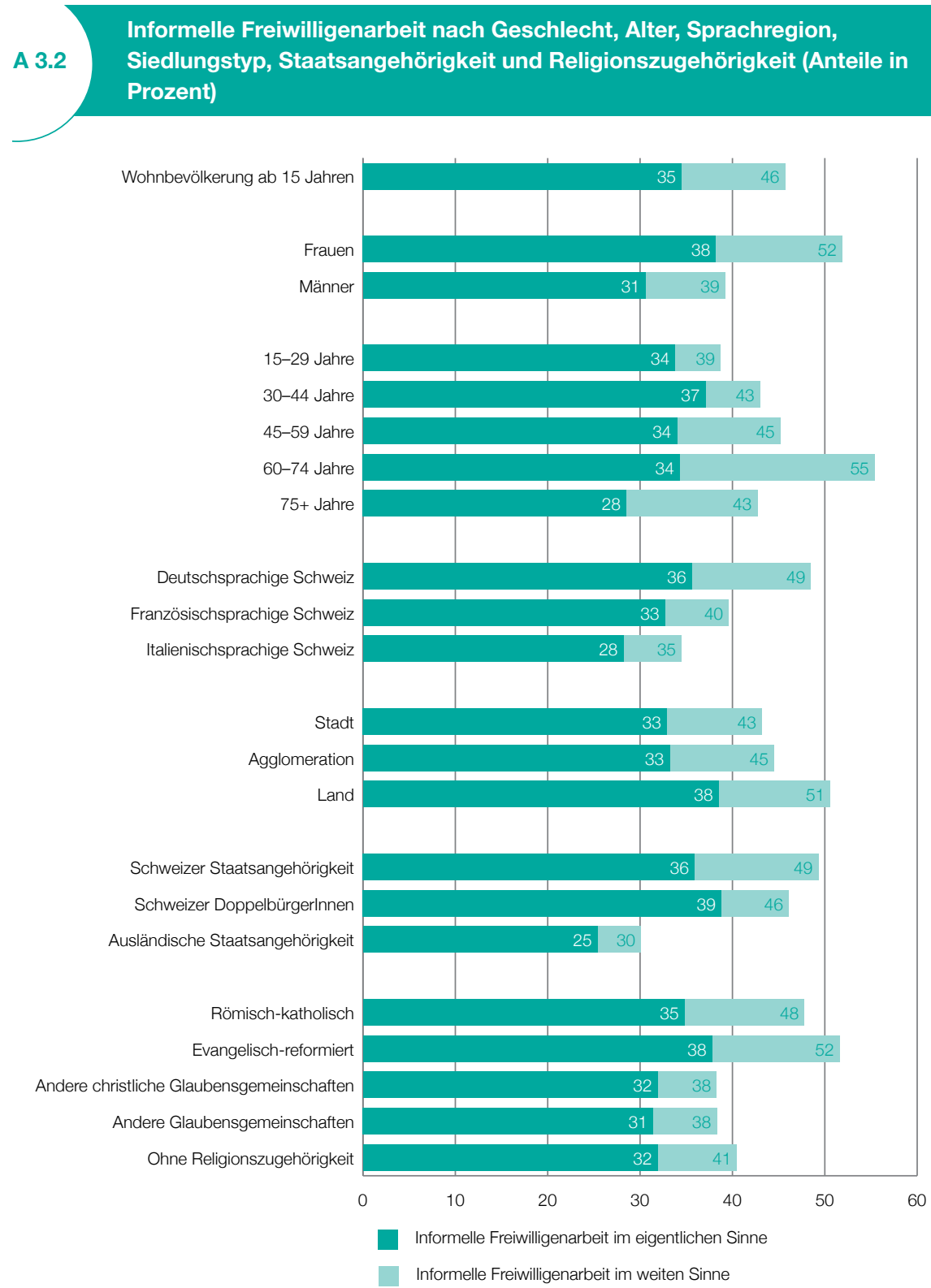

Anzahl Befragte: 4971. Lesebeispiel: 55 Prozent der 60- bis 74-Jährigen leisten ausserhalb von Vereinen oder Organisationen Freiwilligenarbeit für verwandte oder nichtverwandte Personen (informelle Freiwilligenarbeit im weiten Sinne).

34 Prozent der 60- bis 74-Jährigen leisten informelle Freiwilligenarbeit, die nichtverwandten Personen zugutekommt (informelle Freiwilligenarbeit im eigentlichen Sinne). 
informelle Freiwilligenarbeit wird auch auf dem Land geleistet. Zwischen Stadt und Agglomeration gibt es dagegen keinen Unterschied mehr.

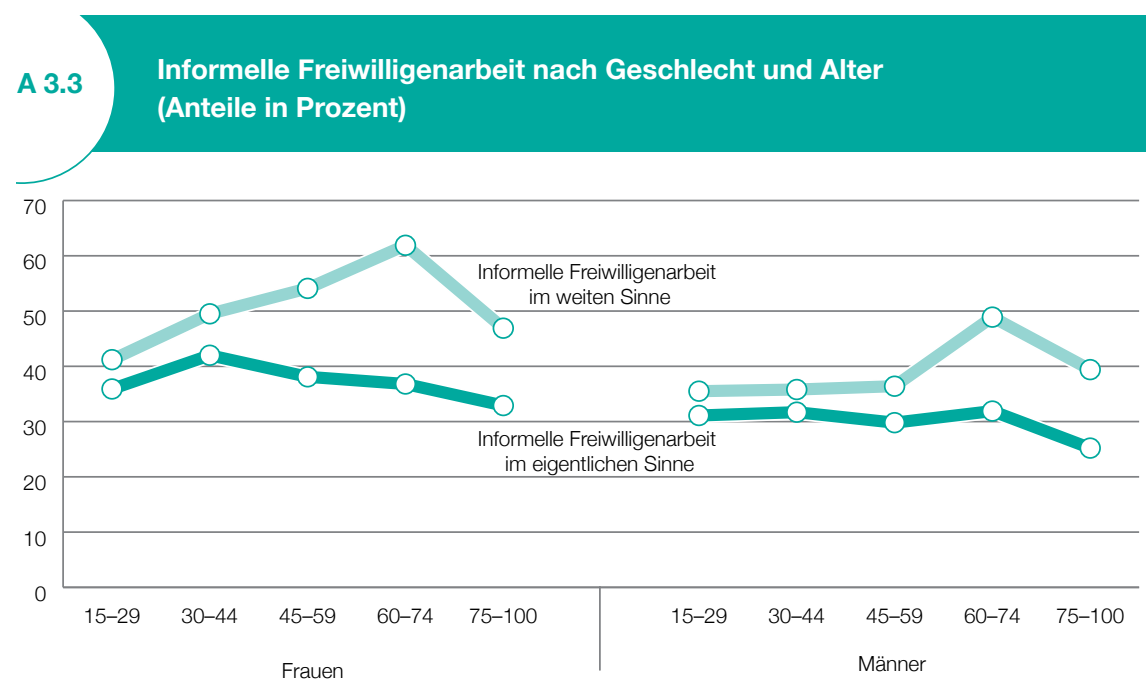

Anzahl Befragte: 4971. Lesebeispiel: 54 Prozent der 45- bis 59-jährigen Frauen leisten ausserhalb von Vereinen oder Organisationen Freiwilligenarbeit für verwandte oder nichtverwandte Personen (informelle Freiwilligenarbeit im weiten Sinne) 38 Prozent der 45- bis 59-jährigen Frauen leisten informelle Freiwilligenarbeit, die nichtverwandten Personen zugutekommt (informelle Freiwilligenarbeit im eigentlichen Sinne).

Einen deutlichen Unterschied gibt es zwischen Personen mit Schweizer Pass und in der Schweiz lebenden Ausländerinnen und Ausländern. Beim Unterschied zwischen den Religionsgemeinschaften scheint es sich ebenfalls um einen versteckten Nationalitäteneffekt zu handeln. Vergleicht man nur Personen mit Schweizer Pass, gleichen sich die Unterschiede zwischen den Religionsgemeinschaften bei der informellen Freiwilligenarbeit im eigentlichen Sinne an. Dass die in der Schweiz lebenden Ausländerinnen und Ausländer weniger informelle Freiwilligenarbeit leisten, kann vor allem auch durch das kleinere soziale Netzwerk in der Schweiz erklärt werden. Viele Bekannte und Verwandte der Migrantinnen und Migranten leben weit weg im Herkunftsland. Hilfeleistungen in den vier Wochen vor dem Interview sind deshalb unwahrscheinlicher. Es liegt also weniger an der Bereitschaft der Ausländerinnen und Ausländern als vielmehr an den Möglichkeiten, 
verwandten und bekannten Personen permanent mit Hilfeleistungen zur Seite zu stehen.

\section{Die unterschiedlichsten Bevölkerungsgruppen engagieren sich informell freiwillig}

Abbildung 3.4 zeigt, wie die Bereitschaft, informelle Freiwilligenarbeit zu leisten, mit sozioökonomischen Merkmalen und der Haushaltsform zusammenhängt. Einige Befunde wollen wir dabei hervorheben:

Personen mit nur obligatorischem Schulabschluss leisten insgesamt weniger informelle Freiwilligenarbeit, wobei diese Gruppe klein ist und es sich dabei relativ oft um ältere oder zugewanderte Personen handelt.

Unter den AkademikerInnen findet man etwas weniger informell Freiwillige im weiten Sinne, was daran liegt, dass sie sich etwas weniger an den Betreuungs- und Pflegearbeiten für verwandte Personen beteiligen. Personen mit Universitätsabschluss leisten mehr formelle Freiwilligenarbeit und werden häufig in ein Amt gewählt (vgl. Abschnitt 2.2). Ansonsten zeigen sich keine nennenswerten Bildungsunterschiede. Auch beim Haushaltseinkommen und bei der beruflichen Stellung sind die Unterschiede weniger ausgeprägt als bei der formellen Freiwilligenarbeit. Einzig die Selbstständigen ohne Angestellte fallen beim formellen wie beim informellen Engagement als überdurchschnittlich aktiv auf. Die grössten Unterschiede finden wir beim Erwerbsstatus: Teilzeiterwerbstätige und Hausfrauen bzw. Hausmänner engagieren sich besonders häufig informell freiwillig. Unabhängig davon, ob sie vollzeit- oder teilzeitangestellt sind, engagieren sich Frauen häufiger informell freiwillig als Männer.

Die Pensionierten erbringen besonders viele Hilfeleistungen für verwandte Personen, vor allem Enkelhüten und Betreuen der betagten Eltern. Bemerkenswert ist schliesslich der grosse Anteil an informell Freiwilligen im eigentlichen Sinne bei Paaren mit Kindern zwischen 6 und 15 Jahren. Sie scheinen sich häufig nicht nur um die eigenen Kinder, sondern auch um Kinder aus der Nachbarschaft und dem sozialen Umfeld ihrer Kinder zu kümmern.

Zusammenfassend kann man sagen, dass sich das informelle Freiwilligenengagement nicht stark zwischen verschiedenen Bildungs-, Berufs-und Einkommensgruppen sowie der Haushaltsform unterscheidet. Die wichtigsten 

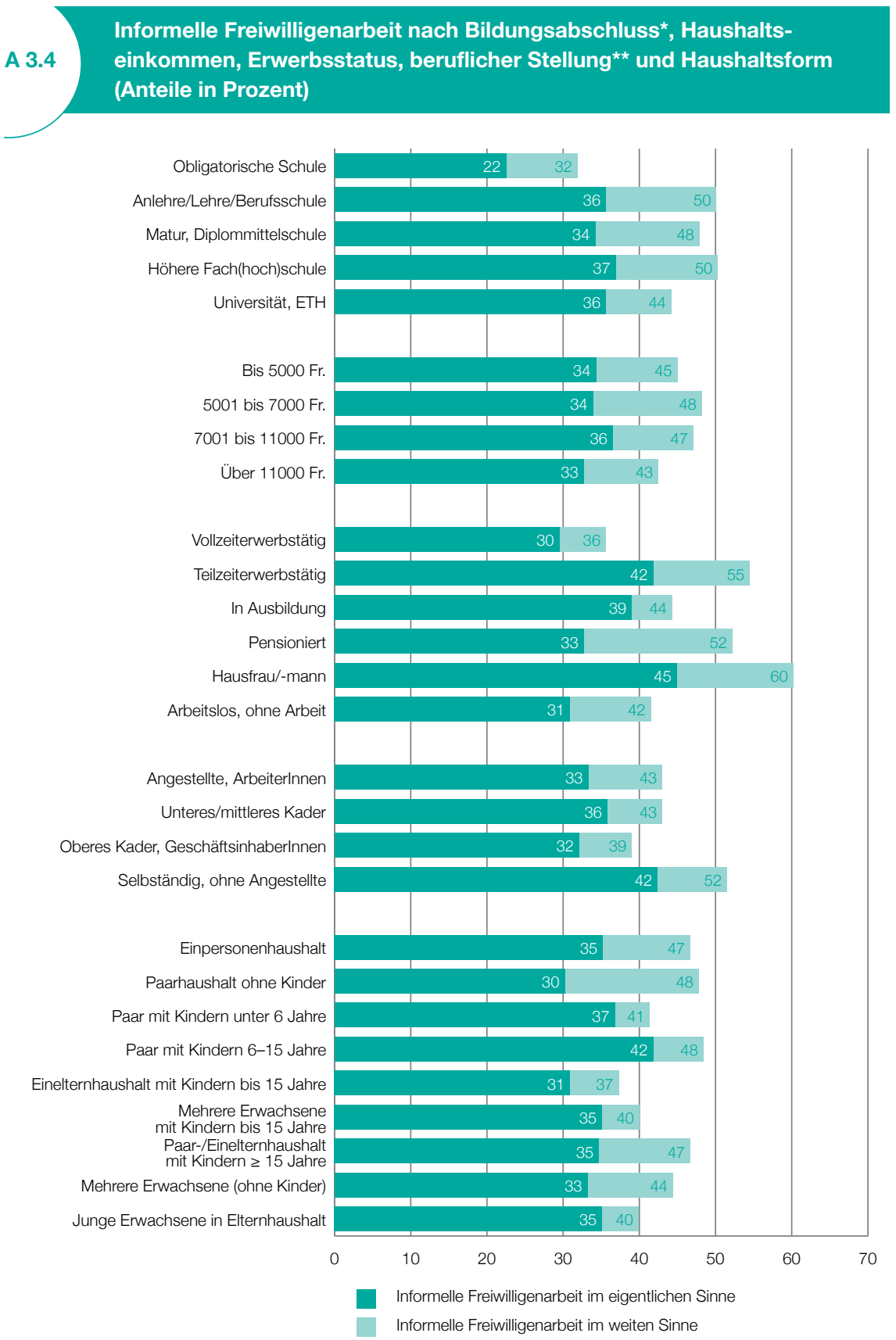

Anzahl Befragte: 4971. Hinweise: ${ }^{*}$ Höchste abgeschlossene Bildung: nur Personen $\geq 30$ Jahre $(\mathrm{N}=4128) .{ }^{\star \star}$ Berufliche Stellung: nur erwerbstätige Personen ( $\mathrm{N}=3389)$. Lesebeispiel: 36 Prozent der vollzeiterwerbstätigen Personen leisten ausserhalb von Vereinen oder Organisationen Freiwilligenarbeit für verwandte oder nichtverwandte Personen (informelle Freiwilligenarbeit im weiten Sinne). 30 Prozent leisten informelle Freiwilligenarbeit, die nichtverwandten Personen zugutekommt (informelle Freiwilligenarbeit im eigentlichen Sinne). 
Einflussfaktoren bleiben das Geschlecht und die Staatszugehörigkeit. Unter informeller Freiwilligenarbeit werden die unterschiedlichsten Formen von Hilfeleistungen zusammengefasst. Dies bietet den unterschiedlichen Bevölkerungsgruppen eine Möglichkeit, sich daran zu beteiligen. Gleichzeitig verschwimmen dabei die Abgrenzungen zur unbezahlten Care-Arbeit. Zukünftig muss entschieden werden, ob die Pflege- und Betreuungsaufgaben gegenüber verwandten Personen ausserhalb des Haushaltes, die in der Kategorie Freiwilligenarbeit im weiten Sinne enthalten sind, überhaupt zur Freiwilligenarbeit zu zählen sind oder ob das vielzitierte Enkelhüten und das Betreuen betagter Eltern als Engagement im privaten Umfeld gesondert erfasst werden müsste. Während die unbezahlte Care-Arbeit für verwandte Personen ausserhalb des eigenen Haushalts in den bisherigen FreiwilligenMonitoren und in der Schweizerischen Arbeitskräfteerhebung (SAKE) in der informellen Freiwilligenarbeit enthalten war, wird sie im deutschen Freiwilligensurvey nicht zur Freiwilligenarbeit gezählt (Simonson et al. 2016). 



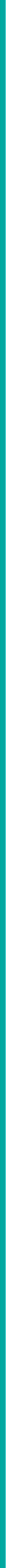


1 Freiwilligkeit

2 Formelle Freiwilligenarbeit und Ehrenamt

3 Informelle Freiwilligenarbeit

$4 \begin{aligned} & \text { Spenden und weitere Formen } \\ & \text { von Freiwilligkeit }\end{aligned}$

5 Motive, Mobilisierung und Potenzial

(6)

Politisches Engagement, Vertrauen und die Freiwilligkeit

Untersuchungsmethode und Stichprobe

Literaturverzeichnis 



\subsection{Spendentätigkeit}

\section{Spenden, die häufigste Form von Freiwilligkeit}

Neben der formellen und informellen Freiwilligenarbeit ist auch das Spenden von Geld und Naturalien eine Form von Freiwilligkeit (vgl. Abschnitt 1.1). Im vorliegenden Monitor geben 71 Prozent der Bevölkerung an, in den vorangehenden zwölf Monaten Geld gespendet zu haben. Dies entspricht exakt dem Anteil aus dem Jahr 2014 (Freitag et al. 2016). Tabelle 4.1 zeigt, dass die gespendete Summe dabei leicht angestiegen ist. 2014 spendeten 30 Prozent der Bevölkerung 300 Franken und mehr, aktuell sind es 36 Prozent. Zehn Prozent der Bevölkerung spendeten 2014 einen Betrag von über 1 ooo Franken spendeten, heute sind es 12 Prozent.

\begin{tabular}{|c|c|c|c|c|c|}
\hline \multirow[t]{3}{*}{ T 4.1} & \multicolumn{5}{|c|}{$\begin{array}{l}\text { Spendenhöhe pro Jahr (Anteil an allen Spendenden und Anteil in der } \\
\text { Bevölkerung ab } 15 \text { Jahren, in Prozent) }\end{array}$} \\
\hline & & \multicolumn{2}{|c|}{2014} & \multicolumn{2}{|c|}{2019} \\
\hline & & $\begin{array}{l}\text { Anteil an allen } \\
\text { Spendenden }\end{array}$ & $\begin{array}{l}\text { Anteil in der } \\
\text { Bevölkerung }\end{array}$ & $\begin{array}{l}\text { Anteil an allen } \\
\text { Spendenden }\end{array}$ & $\begin{array}{l}\text { Anteil in der } \\
\text { Bevölkerung }\end{array}$ \\
\hline \multicolumn{2}{|c|}{ Bis 99 Franken } & 24 & 17 & 19 & 13 \\
\hline \multicolumn{2}{|c|}{ 100-299 Franken } & 33 & 24 & 32 & 22 \\
\hline \multicolumn{2}{|c|}{ 300-499 Franken } & 14 & 10 & 16 & 13 \\
\hline \multicolumn{2}{|c|}{ 500-999 Franken } & 14 & 10 & 15 & 11 \\
\hline \multicolumn{2}{|c|}{ 1000-4999 Franken } & 11 & 8 & 14 & 10 \\
\hline \multicolumn{2}{|c|}{5000 Franken oder mehr } & 4 & 2 & 4 & 2 \\
\hline \multicolumn{2}{|l|}{ Total } & 100 & 71 & 100 & 71 \\
\hline
\end{tabular}

Anzahl Befragte: 2014: 3635; 2019: 3397.

\section{Am häufigsten wird für Umwelt, Natur und Tiere gespendet}

An wen die Spenden hauptsächlich flossen, zeigt die Abbildung 4.1. Am häufigsten wurde für die Bereiche Umwelt, Natur und Tiere (43\% aller Spendenden), Armutsbetroffene im Ausland (40 \%) und Bekämpfung von Krankheiten 
bzw. Pflege und Unterstützung von Kranken (38\%) gespendet. Mindestens je ein Fünftel der Spendenden berücksichtigten zudem Armutsbetroffene in der Schweiz, Menschen mit Behinderung sowie die Katastrophenhilfe. Gegenüber 2014 springt vor allem ins Auge, dass deutlich mehr Spendende den Bereich Umwelt, Natur und Tiere unterstützen. Zusätzlich variiert auch die Spendenhöhe je nach Bereich. Bei Spendensummen von 1 ooo Franken und mehr werden überproportional häufig Armutsbetroffene im Ausland und in der Schweiz sowie Opfer von Katastrophen bedacht.

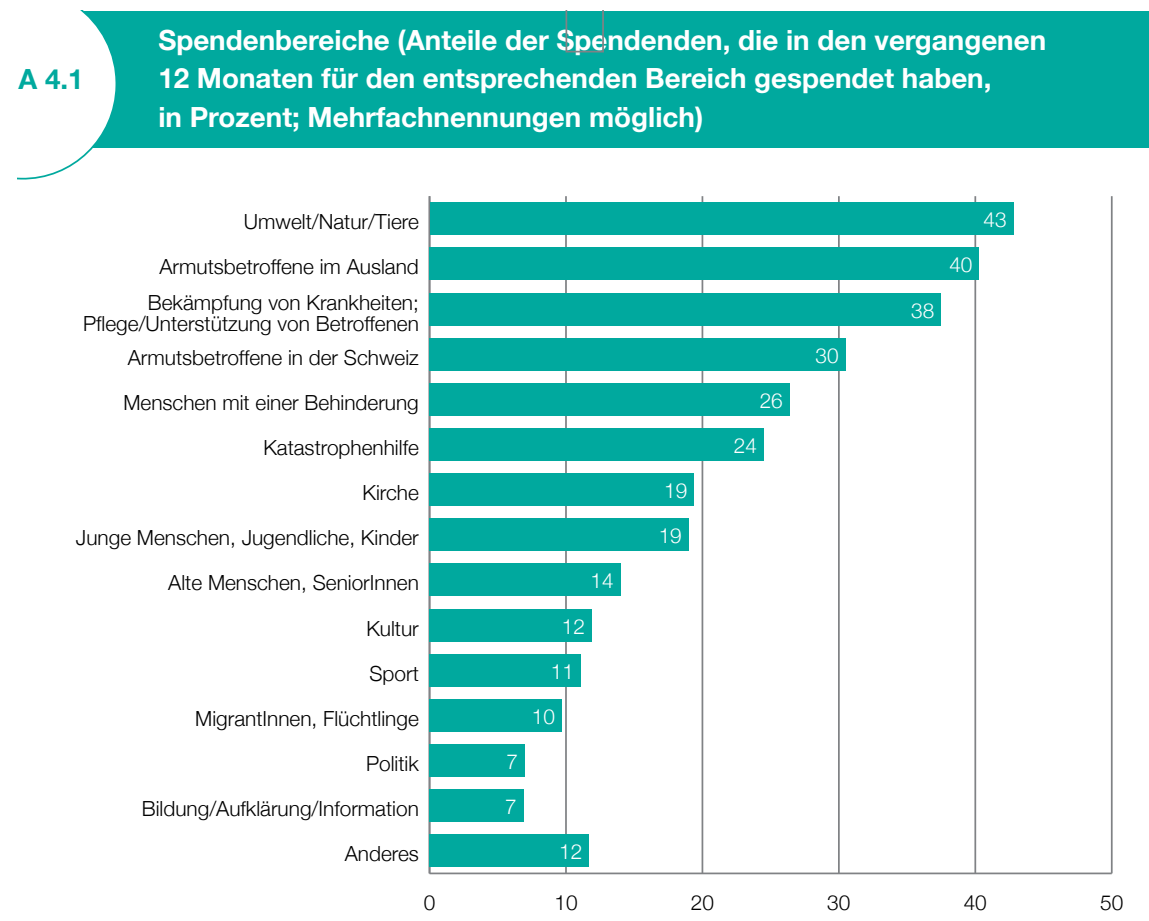

Anzahl Befragte: 3495.

\section{Crowdfunding als neue Form des Spendens}

Eine neue Form der Geldbeschaffung ist das Crowdfunding. Mit Crowdfunding werden innovative Projekte, Produkte oder Unternehmen finanziert, indem möglichst viele Personen sich via Internet direkt an der Geldbeschaffung 
beteiligen und dafür häufig eine nicht-fınanzielle Gegenleistung - ein kleines Dankeschön, ein dabei entstandenes Produkt oder eine entsprechende Dienstleistung-erhalten. Wenn die Gegenleistung entfällt oder mehr ideeller Natur ist, kann Crowdfunding als eine neue Form des Spendens und somit als freiwillige Tätigkeit gesehen werden. 7 Prozent der Befragten geben im Monitor an, in den letzten zwölf Monaten Geld via Crowdfunding für soziale oder gemeinnützige Zwecke gespendet zu haben. Wenn jüngere Personen spenden, nutzen sie relativ häufig Crowdfunding.

\section{Sieben Prozent der Bevölkerung spenden Blut}

Neben den Geldspenden und dem Crowdfunding wurde auch das Blutspenden erfragt. 7 Prozent der Bevölkerung haben in den letzten zwölf Monaten Blut gespendet. Von den 93 Prozent, die kein Blut spenden, sagen 22 Prozent, dass sie kein Blut spenden dürfen. Als ErstspenderInnen werden in der Schweiz nur Personen unter 6o Jahren zugelassen, bei Wiederholungsspendern Personen bis 68 Jahre. Unter den Blutspendenden hat es überdurchschnittlich viele Männer (9\% aller Männer spenden Blut), Personen in Ausbildung (12 \%) und Vollzeiterwerbstätige (11 \%). Die in der Schweiz lebenden Ausländerinnen und Ausländer spenden seltener Blut als die einheimische Bevölkerung: 9 Prozent der Schweizerinnen und Schweizer, 6 Prozent der Doppelbürgerinnen und Doppelbürger sowie 3 Prozent der Ausländerinnen und Ausländer spenden Blut. Die Herkunftsregion der Migrantinnen und Migranten ist dabei kein zusätzlicher Erklärungsfaktor. Auch der Wohnort hat keinen signifikanten Einfluss auf die Bereitschaft, Blut zu spenden. In der Stadt ist das Blutspenden ähnlich verbreitet wie in der Agglomeration und auf dem Land.

\subsection{Das soziale Profil der Spenderinnen und Spender}

Das soziale Profil der Personen, die Geld spenden, lässt sich den Abbildungen 4.2 und 4.3 entnehmen. Frauen spenden etwas häufiger Geld als Männer, Letztere spenden dafür die etwas höheren Summen. Die Bereitschaft, und 
wahrscheinlich auch die Möglichkeit, Geld zu spenden, nimmt mit dem Alter zu. Die Spendenbereitschaft ist auf dem Land, in der Agglomeration und in der Stadt praktisch gleich hoch, in der deutschsprachigen Schweiz aber etwas höher als in der französisch-und italienischsprachigen Schweiz. Höher ist die Spendenbereitschaft auch in der einheimischen Bevölkerung im Vergleich zu den in der Schweiz lebenden Ausländerinnen und Ausländern. Der «Nationalitäteneffekt» beeinflusst zudem den Zusammenhang mit der Religionszugehörigkeit. Betrachtet man nur die einheimische Bevölkerung, so verschwinden die Unterschiede zwischen den verschiedenen christlichen Konfessionen, nicht aber derjenige zwischen den christlichen und den nichtchristlichen Religionen sowie den Konfessionslosen.

\section{Spendenfreudigkeit wächst mit dem verfügbaren Einkommen}

Mit Blick auf den Einfluss der sozioökonomischen Merkmale auf das Spendenverhalten fallen die Unterschiede bei der Erwerbstätigkeit und der Haushaltsform auf. Bei Jüngeren und Personen in Ausbildung ist die Bereitschaft und vermutlich auch die Möglichkeit zu spenden deutlich tiefer. Gleiches gilt für Personen ohne Arbeit und Personen mit Kindern - insbesondere wenn es sich dabei um Alleinerziehende oder Patchworkfamilien handelt. Etwas lapidar ausgedrückt könnte man sagen: Dort wo das Geld eher knapp ist, bleibt weniger zum Spenden übrig. Dies bestätigt sich mit Blick auf das Haushaltseinkommen, indem die Spendenfreudigkeit mit der Höhe des Einkommens steigt. Allerdings ist der Anteil an Spendenden selbst in der untersten Einkommenskategorie mit 62 Prozent immer noch beträchtlich, und die Unterschiede zwischen den mittleren und hohen Einkommen sind nur noch klein. Mit der Höhe des Einkommens steigt tendenziell auch die Höhe der Spende. Bei etwa zwei Drittel der Spendenden aus der tiefsten Einkommenskategorie sind die jährlichen Spenden nicht höher als zoo Franken, bei denjenigen der höchsten Einkommenskategorie gilt dies für 40 Prozent. Wenn man allerdings den Anteil der Spende am Einkommen anschaut, so präsentieren sich die GrossverdienerInnen nicht grosszügiger als die Personen mit kleinem Lohn. 


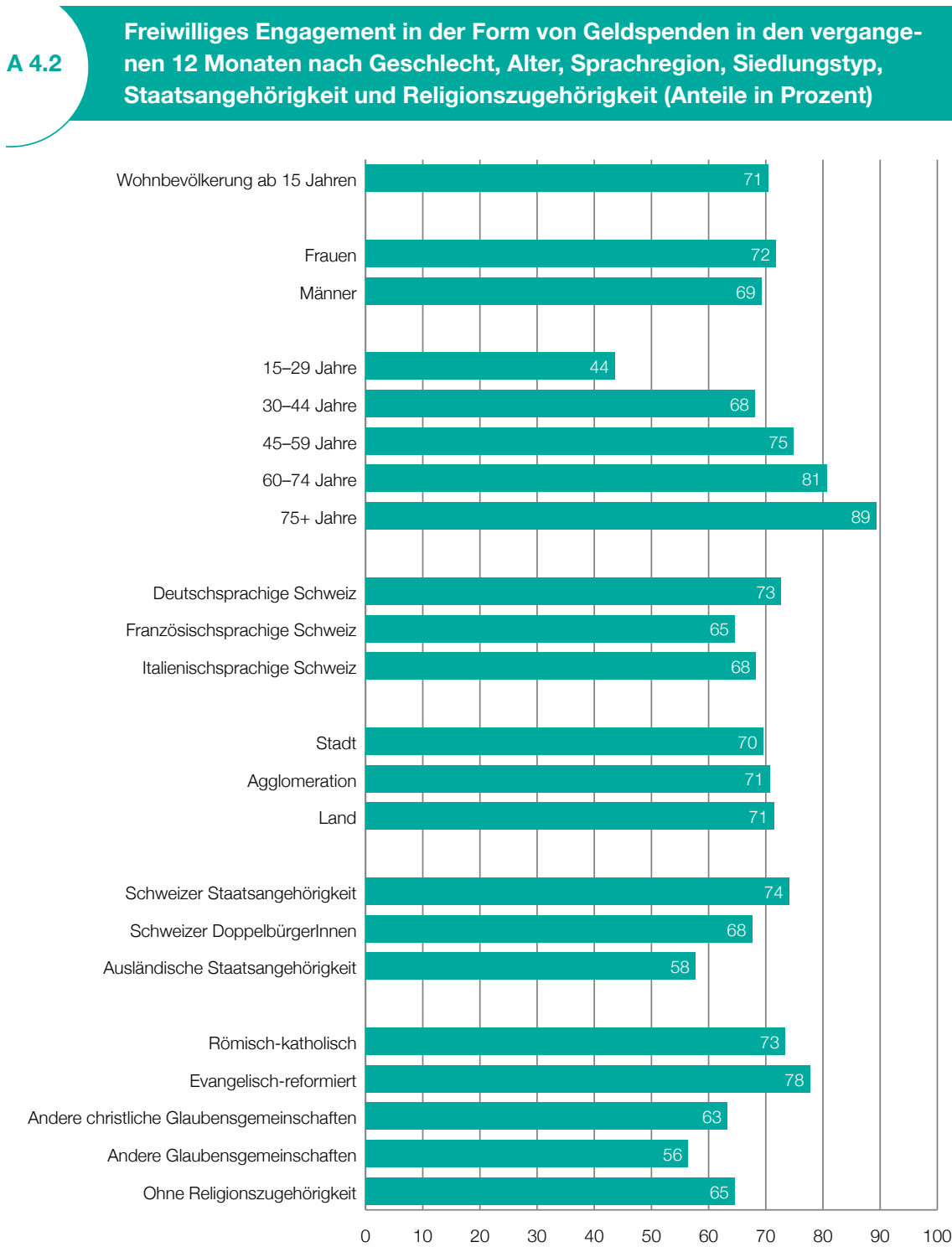

Anzahl Befragte: 4987. 

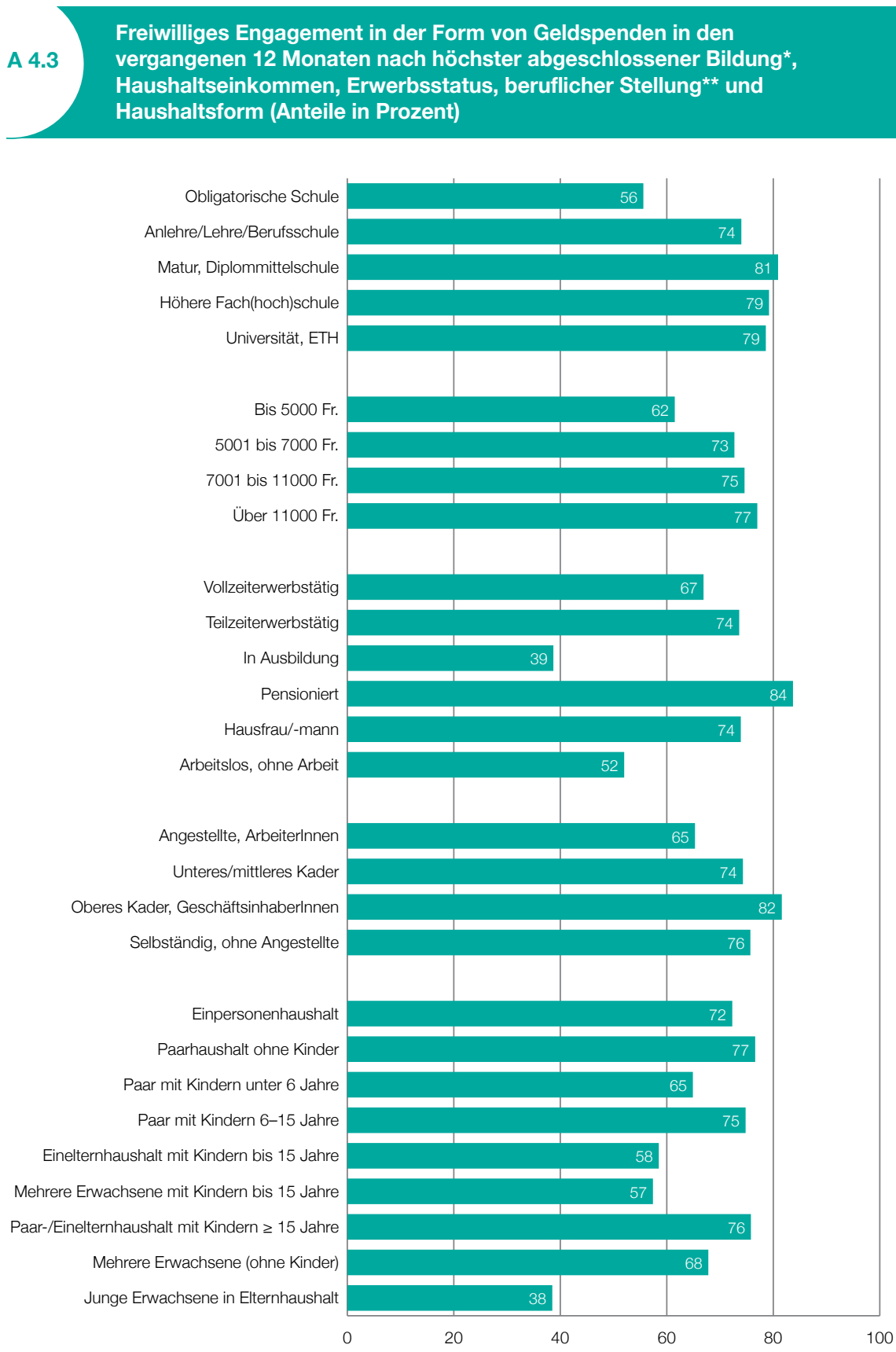

Anzahl Befragte: 4987. Hinweise: * Höchste abgeschlossene Bildung: nur Personen $\geq 30$ Jahre $(\mathrm{N}=4142) .{ }^{* \star}$ Berufliche Stellung: nur erwerbstätige Personen $(\mathrm{N}=3395)$. 
Bei den Bildungsunterschieden fällt nur die kleine Gruppe der Personen ab, die lediglich die obligatorische Schule besucht haben. Ansonsten findet man im Spendenverhalten von Personen mit mittlerer und hoher Bildung keine grossen Unterschiede.

\section{Ältere Personen spenden besonders häufig für die junge Generation}

Frauen und Männer sowie ältere und jüngere Personen haben nicht grundsätzlich andere Präferenzen beim Spenden. Bei beiden Geschlechtern und in allen Altersgruppen liegen Umwelt, Natur und Tiere sowie Armutsbetroffene im Ausland an erster Stelle. Interessant ist aber, dass ältere Menschen deutlich häufiger für junge Menschen, Jugendliche und Kinder spenden sowie für die Bekämpfung von Krankheiten, für die Pflege von Kranken und für Menschen mit Behinderungen. Frauen spenden etwas häufiger für Umwelt, Natur und Tiere, für junge Menschen und für die Kirche, während Männer öfters für die Katastrophenhilfe, für Sport und für Politik spenden. Die in der Schweiz lebenden Ausländerinnen und Ausländer bedenken mit ihren Spenden vermehrt Armutsbetroffene im Ausland sowie die Bekämpfung von Krankheiten und die Pflege von Kranken, wohingegen Umwelt, Natur und Tiere sowie die Armutsbetroffenen in der Schweiz bei ihnen eine tiefere Priorität haben.

\subsection{Freiwilliges Engagement im Internet}

\section{Internet-Freiwillige sind überwiegend junge, männliche Städter}

Im Monitor 2016 wurde erstmals das freiwillige Engagement im Internet untersucht. Das Internet wurde dabei als «zusätzliche Arena des sozialen Austauschs verstanden, die zahlreiche Möglichkeiten zur Freiwilligkeit eröffnet» (Freitag et al. 2016, 124). Im neuesten Monitor wurden die Fragen zum Freiwilligenengagement im Internet weiter ausgebaut. Dabei zeigt sich, 
dass 13 Prozent der Bevölkerung die Möglichkeiten des Internets nutzen, um sich freiwillig und unentgeltlich zu engagieren. Der Anteil liegt aber deutlich unter den 25 Prozent Internet-Freiwilligen aus dem Jahr 2014. Dieser Unterschied kann damit erklärt werden, dass 2014 gefragt wurde, ob man sich jemals engagiert habe, während 2019 das Engagement in den letzten zwölf Monaten erfasst wurde.

Wie 2014 zeigen sich auch aktuell signifikante Unterschiede nach Geschlecht, Alter und Wohnort. Männer engagieren sich mehr als Frauen (16\% versus $10 \%$ ), unter 45-Jährige mehr als über 45-Jährige (18\% versus $10 \%$ ) und StädterInnen mehr als die Landbevölkerung (15\% versus $11 \%$ ).

\section{Das Internet als willkommenes Hilfsmittel und Medium in der Freiwilligenarbeit}

Um was für ein Engagement es sich bei der Internet-Freiwilligkeit handelt, kann Tabelle 4.2 entnommen werden. Am häufigsten - nämlich von 32 Prozent der Internet-Freiwilligen oder von 4.3 Prozent der Bevölkerung - wird die Internetseite eines Vereins oder einer Organisation bewirtschaftet. Jeweils gut ein Fünftel der Internet-Freiwilligen gründete oder moderiert eine Gruppe in einem sozialen Netzwerk (z. B. eine Facebook- oder WhatsApp-Gruppe) oder postet und verbreitet Informationen von gemeinnützigen Initiativen. Der Blick auf die verschiedenen Formen der Internet-Freiwilligkeit in Tabelle 4.2 macht deutlich, dass es sich bei der Mehrheit der Tätigkeiten um Aktivitäten handelt, die sich auf eine realweltliche Freiwilligenarbeit beziehen und bei denen das Internet als Arbeitsmittel oder Medium eingesetzt wird. Reine Internet-Freiwilligkeit wie etwa Einträge bei «open street map» und Wikipedia erstellen oder in einem Open-Source-Projekt mitarbeiten, kommen zwar vor, sie sind aber relativ selten.

Diese Beobachtung bestätigt sich, wenn wir die Internet-Freiwilligen fragen, für wen sie sich im Internet engagieren. 65 Prozent der InternetFreiwilligen sind im Internet für eine Gruppe oder einen Verein tätig, deren Aktivitäten hauptsächlich ausserhalb des Internets stattfinden. Nur ein Fünftel (19\%) engagiert sich für eine virtuelle Gruppe, die vor allem im Internet existiert. 8 Prozent geben einen anderen Bezugsrahmen ihrer InternetFreiwilligkeit an und 29 Prozent engagieren sich im Internet einfach für sich 
selbst oder ihre Familie. Da in diesem Fall das Drittpersonen-Kriterium nicht erfüllt ist und sich das Engagement auf das familiäre Umfeld beschränkt, können wir hier nicht mehr von Freiwilligenarbeit sprechen.

T 4.2 Formen der Freiwilligentätigkeit im Internet (Anteile an allen InternetFreiwilligen und in der Bevölkerung ab 15 Jahren, in Prozent)

\begin{tabular}{lcc} 
& $\begin{array}{c}\text { Anteil an allen } \\
\text { Internet- } \\
\text { Freiwilligen }\end{array}$ & $\begin{array}{c}\text { Anteil in der } \\
\text { Bevölkerung }\end{array}$ \\
\hline Homepage von Vereinen/Organisationen bewirtschaften & 32 & 4.3 \\
Gruppe in sozialem Netzwerk gründen/moderieren & 21 & 2.8 \\
Informationen von gemeinnützigen Organisationen posten/verbreiten & 21 & 2.8 \\
Informative Forenbeiträge, Blog verfassen & 19 & 2.5 \\
Beratung und Expertise anbieten & 18 & 2.4 \\
Informationsdokumente für Online-Verbreitung verfassen (z. B. Newsletter) & 15 & 2.0 \\
Eintrag auf «open street map» verfassen & 8 & 1.0 \\
Beiträge in Online-Enzyklopädien verfassen (z. B. Wikipedia) & 8 & 1.0 \\
Bedienungsanweisungen/Testberichte auf Internet-Portalen veröffentlichen & 6 & 0.8 \\
Bei Open-Source-Projekten mitarbeiten (z. B. Linux, OpenOffice) & 100 & 0.8 \\
Forum moderieren & 6 & 13.0 \\
Crowdfunding-Aktion für ein gemeinnütziges Projekt lancieren & 3 & 0.5 \\
Bei digitalen Citizen-Science-Projekten mitmachen & 3 & 0.4 \\
Anderes & 2 & 0.2 \\
\hline Total & 3 & 1.6 \\
\hline
\end{tabular}

Anzahl Befragte: 657. Lesebeispiel: 32 Prozent der Personen, die sich im Internet freiwillig engagieren, bewirtschaften dabei die Homepage eines Vereins oder einer Organisation. Bezogen auf die Bevölkerung sind es 4.3 Prozent, die eine solche Tätigkeit ausüben.

\section{Stundeneinsatz der Internet-Freiwilligen}

Im Durchschnitt engagieren sich die Internet-Freiwilligen 1.25 Stunden pro Woche freiwillig im Internet. Bei der Hälfte davon ist es höchstens eine halbe Stunde, während sich 5 Prozent fünf Stunden oder mehr engagieren. Dieses hohe Engagement findet sich vor allem bei Personen, die sich in mehreren Formen im Internet engagieren und beispielsweise neben der Bewirtschaftung einer Internetseite eines Vereins auch noch einen Blog betreiben, Beratung 
und Expertise anbieten, Gruppen moderieren oder Beiträge in OnlineEnzyklopädien verfassen.

Relativ häufig ist die aufgewendete Zeit bereits in die Angaben zum freiwilligen Engagement innerhalb von Vereinen oder Organisationen eingeflossen (vgl. Abschnitt 2.2). Immerhin 37 Prozent der Internet-Freiwilligen geben aber an, dass ihr Engagement im Internet nicht Teil ihrer sonstigen Freiwilligentätigkeit ist und die Zeit, die sie dafür aufwenden, zum restlichen Engagement dazugezählt werden muss.

\subsection{Sharing Economy}

\section{Sharing Economy: Geschäftsmodell oder neue Form von Freiwilligkeit?}

Immer mehr Güter und Dienstleistungen, von Haushaltsgeräten und Werkzeugen über Velos und Autos bis hin zu Wohnungen und Ferienhäusern, werden heute über digitale Plattformen getauscht und geteilt. Ermöglicht hat diese Sharing Economy die Digitalisierung bzw. die Verbreitung des Internets und mobiler Zugangsgeräte (vgl. von Stokar et al. 2018). Der Begriff «Sharing Economy» nennt es beim Namen: Entstanden ist eine neue Form von Ökonomie mit gewinnbringenden Geschäftsmodellen und mächtigen AkteurInnen wie Uber, Airbnb oder Spotify, die als Sharing-Economy-Plattformen zu Milliardenunternehmen geworden sind. Vieles, was heute unter Sharing Economy verkauft bzw. geteilt wird, hat weder mit «Freiwilligkeit» noch mit «Non-Profit» zu tun. Vielmehr handelt es sich um ein Geschäftsmodell, bei dem sich MarktteilnehmerInnen über digitale Plattformen finden, um Güter und Dienstleistungen zu verkaufen und zu kaufen (Eckhardt und Bardhi 2015).

Sharing Economy kann aber dann als eine Form von Freiwilligkeit gesehen werden, wenn man bereit ist, persönliche Dinge mit anderen Personen ausserhalb des familiären Umfelds unentgeltlich zu teilen oder auf einem digitalen Marktplatz kostenlos oder sehr günstig anzubieten. Diese Bereitschaft wurde im vorliegenden Monitor erstmals erhoben. Abbildung 4.4 gibt einen Überblick über die verschiedenen Dinge, welche die Befragten 
unentgeltlich teilen würden, und zeigt, mit wem man was teilen würde. Bei den Antworten muss berücksichtigt werden, dass die Befragten häufig keine Einschätzung abgaben, weil sie diese Dinge nicht besitzen oder auf die Fragen keine Antwort wussten. Am häufigsten ist das bei der Ferienwohnung (58\%), beim Fahrrad oder E-Bike (28\%) und beim Auto (23\%) der Fall.

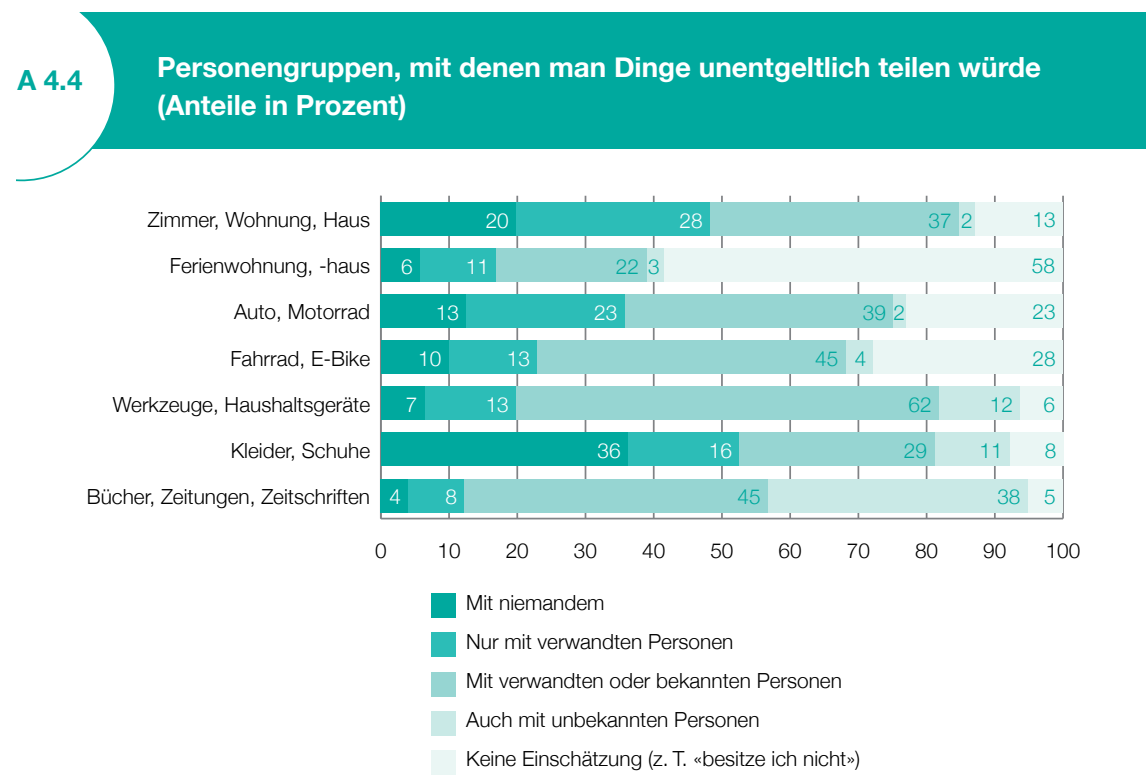

Anzahl Befragte: 4947. Lesebeispiel: 10 Prozent der Befragten würden ihr Fahrrad oder E-Bike nicht unentgeltlich teilen, wenn sie es nicht selber nutzen, 13 Prozent nur mit verwandten Personen, 45 Prozent mit verwandten oder bekannten Personen und 4 Prozent auch mit unbekannten Personen. 28 Prozent nehmen keine Einschätzung vor, vielfach, weil sie kein Fahrrad oder E-Bike besitzen.

\section{Je persönlicher ein Gegenstand, desto geringer die Bereitschaft zu teilen}

91 Prozent der Schweizer Bevölkerung sind bereit, ihre Bücher, Zeitungen und Zeitschriften mit anderen Leuten zu teilen. Diese Printprodukte würde ein hoher Anteil der Bevölkerung auch mit unbekannten Personen teilen (38 \%). Bei allen anderen in Abbildung 4.4 dargestellten Gütern - wie Werkzeuge, Fahrräder oder Auto und Wohnung - ist zwar eine klare Mehrheit bereit zu teilen, aber nur mit Personen, die sie persönlich kennen. Zurückhaltendend 
ist man beim Teilen von Kleidern und Schuhen. 36 Prozent der Bevölkerung möchten ihre Kleider mit niemandem teilen. Je persönlicher ein Gegenstand ist, desto weniger ist man bereit, diesen zu teilen bzw. desto eher teilt man ihn nur mit Personen, die einem vertraut sind. Insgesamt verweist Abbildung 4.4 auf ein erhebliches, aber je nach Objekt unterschiedliches Potenzial für das freiwillige und unentgeltliche Teilen von Dingen, die man gerade nicht selber nutzt.

\section{Jüngere und Frauen sind offener für die Sharing Economy}

Bei den Frauen ist die Bereitschaft zu teilen leicht höher als bei den Männern. 46 Prozent aller Frauen und 39 Prozent aller Männer würden mindestens etwas aus Abbildung 4.4 mit einer ihnen nicht bekannten Person teilen. Die Bereitschaft zu teilen ist bei den Frauen bei sämtlichen Gegenständen und Gütern höher, wobei der Geschlechterunterschied bei den Kleidern am grössten ist: 45 Prozent der Männer aber nur 28 Prozent der Frauen würden mit niemandem ihre Kleider teilen. Noch grösser als die Geschlechter-sind allerdings die Altersunterschiede. Jüngere sind eher bereit als ältere, persönliche Dinge anderen Personen zu überlassen, wobei sich die Zwanzigjährigen nicht gross von den Dreissigjährigen unterscheiden. Mindestens ein Objekt aus Abbildung 4.4 einer unbekannten Person überlassen, würden über 50 Prozent der 15-bis 40-Jährigen, aber weniger als 30 Prozent der über 6o-Jährigen. Die Altersunterschiede bestehen sowohl bei Buch und Zeitung als auch bei den Werkzeugen, dem Auto oder der Wohnung. 



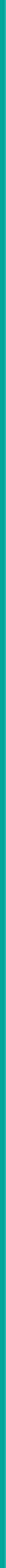


1 Freiwilligkeit

2 Formelle Freiwilligenarbeit

2 und Ehrenamt

3 Informelle Freiwilligenarbeit

4. Spenden und weitere Formen von Freiwilligkeit

5 Motive, Mobilisierung und Potenzial

6

Politisches Engagement, Vertrauen und die Freiwilligkeit

Untersuchungsmethode und Stichprobe

Literaturverzeichnis 



\subsection{Motive und Zufriedenheit der formellen Freiwilligen}

\section{Der Spass steht an erster Stelle}

Im vorliegenden Monitor werden 19 Motive für die Freiwilligenarbeit abgefragt (Abbildung 5.1). Gegenüber 2014 sind neue Motive dazugekommen, die vor allem den Gewinn des Engagements für die Freiwilligen selbst betonen. Dies geschah vor dem Hintergrund, dass Freiwilligenarbeit heute immer weniger altruistisch begründet wird und es nicht primär darum geht, Gutes zu tun, sondern Spass an der Sache zu haben und mit Gleichgesinnten etwas zu bewegen (Braun 2003; Schüll 2006; Wehner und Güntert 2015; Samochowiec et al. 2018). Tatsächlich findet sich die Freude an der Tätigkeit zuoberst auf der Liste der Motive. 7o Prozent der formellen Freiwilligen engagieren sich, weil ihnen die Tätigkeit Spass macht. An zweiter Stelle stehen die sozialen Aspekte von Freiwilligenarbeit. Man will in der Freiwilligenarbeit mit anderen Menschen zusammenkommen, mit ihnen etwas bewegen und dabei auch helfen und etwas zurückgeben. Drittens geht es darum, sich selber weiterzubringen, die eigenen Kenntnisse und Erfahrungen zu erweitern, sich persönlich weiterzuentwickeln und persönliche Netzwerke zu pflegen.

\section{Die finanzielle Entschädigung ist kein Beweggrund}

Während sich immerhin rund ein Viertel der Freiwilligen auch engagiert, weil sie dafür Wertschätzung und Anerkennung erhalten, wird die finanzielle Entschädigung nur selten genannt. Ebenfalls nur selten übt man eine Freiwilligentätigkeit aus, weil es von einem erwartet wird oder weil man dazu gedrängt oder verpflichtet wurde. Erwähnenswert ist, dass selbst Personen, die Entschädigungen von über 20 Franken pro Stunde erhalten, mehrheitlich sagen, dass sie sich nicht wegen der finanziellen Entschädigung engagieren.

Anderen zu helfen und Gutes zu tun ist zwar immer noch wichtig, man macht dies aber weder aus Pflichtgefühl oder religiöser Überzeugung, noch weil es erwartet wird und auch nicht wegen der finanziellen Entschädigung - sondern weil man es gerne tut und weil man es mit anderen zusammen tun kann. 


\section{Unterschiedliche Bereiche - unterschiedliche Motive}

Die Beweggründe von Männern und Frauen für ein freiwilliges Engagement unterscheiden sich nicht gross. Die Frauen betonen etwas mehr, dass sie anderen Menschen helfen und ihre Kenntnisse und Erfahrungen erweitern wollen oder sich aus religiöser und spiritueller Überzeugung engagieren. Die Reihenfolge der Motive in Abbildung 5.1 ändert sich aber nicht nennenswert, wenn wir sie für Frauen und Männer getrennt darstellen. Auch zwischen den verschiedenen Bereichen des Engagements finden sich keine fundamentalen Unterschiede. Es gibt aber einige interessante Abweichungen: Beim Engagement in Selbsthilfegruppen steht selbstredend nicht der Spass im Vordergrund. So engagiert sich nur knapp die Hälfte der Freiwilligen in diesem Bereich aus Spass an der Tätigkeit. Nicht nur in den Sport-, Hobbyund Kulturvereinen, sondern auch in anderen Bereichen ist der Spass hingegen ein wichtiger Antrieb: In den sozialen und karitativen Organisationen, in den Umwelt-und Naturschutzorganisationen, in politischen und öffentlichen Gremien oder Ämtern, im Elternrat, in einer politischen Partei oder im öffentlichen Dienst engagieren sich mindestens 7o Prozent der Freiwilligen, weil ihnen die Tätigkeit Spass macht. Der öffentliche Dienst erreicht beim «Spassfaktor» mit 78 Prozent sogar einen Spitzenwert.

«Anderen Menschen helfen» ist für die Freiwilligen in sozialen und karitativen Organisationen, in Selbsthilfegruppen, in Menschenrechtsorganisationen und im öffentlichen Dienst ein besonders wichtiger Beweggrund (jeweils von über 70 \% genannt), während «Helfen» im kulturellen Verein, im Interessenverband, in den Umwelt- und Naturschutzorganisationen sowie im Sportclub etwas weniger im Vordergrund steht (jeweils von weniger als $50 \%$ genannt). In einem politischen oder öffentlichen Gremium, in einer Jugendorganisation, im öffentlichen Dienst, in einer Menschrechtsorganisation, in einer politischen Partei oder im Elternrat engagiert man sich häufig, weil man seine Kenntnisse und Erfahrungen erweitern will (jeweils von über $50 \%$ als Beweggrund genannt). Und wer Wertschätzung und Anerkennung sucht, scheint im öffentlichen Dienst gut aufgehoben zu sein - und Spass scheint es darüber hinaus auch zu machen. 


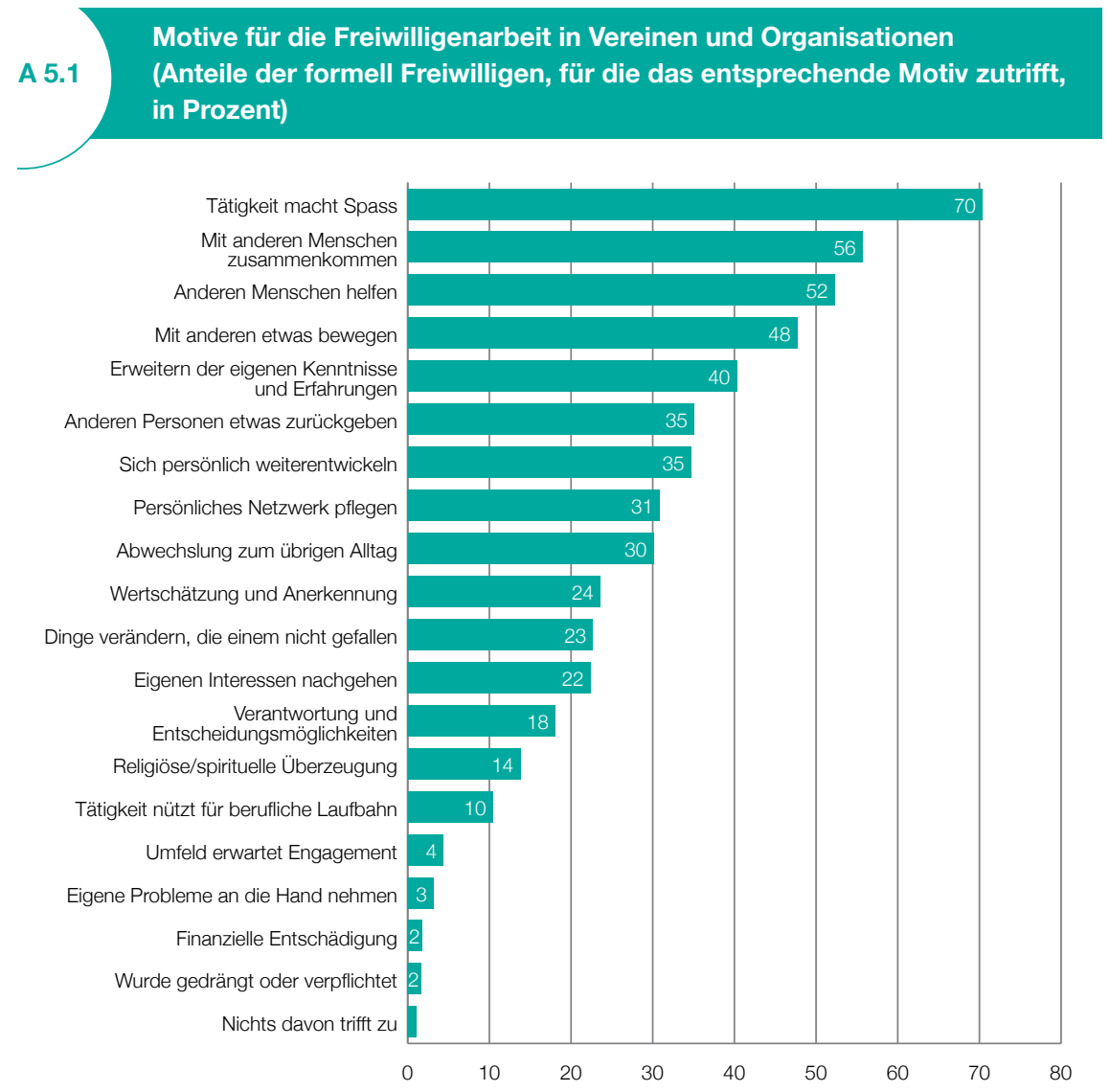

Anzahl Befragte: 1928.

\section{Hohe Zufriedenheit der Ehrenamtlichen}

Die Personen, die ein gewähltes Amt ausüben, wurden zusätzlich gefragt, ob sie dieses Amt nochmals übernehmen würden, wenn sie jetzt wählen könnten. 73 Prozent der Ehrenamtlichen antworten darauf mit «ja, auf jeden Fall», 19 Prozent mit «ja, unter Umständen», 4 Prozent mit «eher nicht» und 1 Prozent mit «nein, auf keinen Fall». Die restlichen 3 Prozent haben eine unterschiedliche Einschätzung je nach Amt. Aus diesen Antworten spricht eine beachtliche Zufriedenheit der Ehrenamtlichen. Zwischen Frauen und Männern gibt es keine signifikanten Unterschiede, und auch die verschiedenen Altersgruppen unterscheiden sich nicht wesentlich. Ebenso wenig 
hängt die Zufriedenheit mit der Tätigkeit zusammen. Unabhängig davon, ob es sich um einen Vereinsvorstand, eine Milizbehörde, einen Stiftungsrat, eine Genossenschaft, eine Kirchenpflege oder die Leitung eines Teams oder einer Gruppe handelt, in allen Bereichen würden mindestens go Prozent der Ehrenamtlichen ihr Amt (unter Umständen) nochmals übernehmen.

\section{Fehlende Zeit und mangelnder Teamgeist als Hauptprobleme}

Interessant ist, welche Gründe dazu führen, dass die Amtsinhaberinnen und Amtsinhaber ihr Amt nicht mehr übernehmen würden. An erster Stelle wird moniert, das Amt brauche zu viel Zeit. 53 Prozent der Ehrenamtlichen, die ihr Amt nicht mehr übernehmen würden, sagen, es sei zu zeitintensiv. Als weitere Gründe folgen «zu wenig Zusammenhalt im Team» (25\%), «andere Interessen und Verpflichtungen» (22\%), «zu wenig Anerkennung» (15\%), «zu viel Bürokratie und Leerlauf» (12\%), «zu viel Verantwortung» (10\%) sowie «zu wenig Mitsprache und Mitbestimmung» (10 \%). Ganz ähnliche Antworten erhält man, wenn man die Ehrenamtlichen, die angeben, sie würden das Amt «unter Umständen» wieder übernehmen, nach diesen Umständen fragt. Auch hier steht an erster Stelle «müsste mehr Zeit haben» (47\%) vor «besserer Zusammenhalt im Team» (30 \%), «weniger andere Verpflichtungen» (29\%), «weniger Bürokratie und Leerlauf» (18\%), «mehr Anerkennung» (11\%), «mehr Mitsprache und Mitbestimmung» (9\%) und «weniger Verantwortung» (5\%).

\section{Durchschnittlich dauert ein formelles Engagement bereits 18 Jahre}

Die Mehrzahl der Freiwilligen engagiert sich schon seit vielen Jahren, wobei die Dauer des Engagements nachvollziehbarerweise vom Alter der Personen abhängt. Im Durchschnitt engagieren sich die Freiwilligen seit 18 Jahren in Vereinen oder Organisationen. Bei einem Viertel sind es mindestens zo Jahre, bei einem Fünftel noch keine 5 Jahre. Betrachtet man das durchschnittliche Engagement in den verschiedenen Bereichen, so sind die Engagierten in politischen Parteien, in Religionsgemeinschaften und Kirchen, in Sportclubs und in kulturellen Vereinen besonders viele Jahre aktiv, während das Enga- 
gement in Selbsthilfegruppen, in politischen oder öffentlichen Ämtern, in Jugendorganisationen, in sozialen und karitativen Organisationen sowie in Elternräten weniger lange dauert. Dies liegt jedoch häufig daran, dass das Durchschnittsalter der Engagierten in diesen Bereichen tiefer liegt (Jugendorganisationen, Elternräte) oder dass das Engagement durch Bestimmungen oder andere Faktoren zeitlich begrenzt ist (politisches oder öffentliches Amt, Elternräte). Die Zufriedenheit mit dem Amt hängt nicht mit der Dauer des Engagements zusammen. Personen, die sich schon lange freiwillig engagieren und ein Ehrenamt ausüben, würden ihr Amt ebenso häufig wieder übernehmen wie Personen, die sich noch nicht so lange freiwillig engagieren.

\section{Ehemals formell Freiwillige berichten von guten Erfahrungen}

Will man mehr über die Situation und Zufriedenheit der Freiwilligen erfahren, so müssen natürlich auch diejenigen zu Wort kommen, die ihr Amt aufgegeben haben. Von den 61 Prozent der Schweizer Bevölkerung, die zur Zeit nicht formell freiwillig tätig sind, hat gut die Hälfte (52 \%) früher einmal Aufgaben oder Arbeiten in einem Verein oder einer Organisation ausgeübt. Für einige liegt dieses Engagement erst ein oder zwei Jahre zurück, für andere über vierzig Jahre. Im Durchschnitt ist man seit gut neun Jahren nicht mehr formell freiwillig tätig. Am häufigsten war ein Engagement in einem Sportverein (41\%) oder in einem kulturellen Verein (22\%). Je über 10 Prozent engagierten sich in einer Religionsgemeinschaft oder Kirche, in einer sozialen oder karitativen Organisation, in einem Spiel- oder Hobbyverein, in einem Gemeinde-, Orts- oder Quartierverein oder in einer Jugendorganisation. Wenn die ehemaligen Freiwilligen ihr früheres Engagement auf einer Skala von o «sehr negative Erfahrungen» bis 10 «sehr positive Erfahrungen» bewerten müssen, so resultiert daraus eine Durchschnittsnote von 7.9. Die überwältigende Mehrheit spricht also von guten oder sehr guten Erfahrungen, nur eine Minderheit von 4 Prozent bewertet das frühere Engagement negativ und gibt eine Note unter 5. Die Bewertungen unterscheiden sich kaum zwischen Geschlecht, Alter, Nationalität oder Bereich. Die höchste Note gibt es von den Engagierten in einer Umwelt-, Natur- oder Tierschutzorganisation (8.3), 
die tiefste von den ehemaligen Engagierten in einem Interessenverband. Aber auch hier liegt der Durchschnittswert bei hohen 7.5.

\section{Die Schwierigkeit, Beruf, Familie und Amt unter einen Hut zu bringen, führt zu Ausstiegen}

Die positiven Erfahrungen der ehemals Freiwilligen widerspiegeln sich auch in den Gründen, weshalb sie ihre Freiwilligenkarriere beendet haben. Die wichtigsten vier Gründe, die für den Ausstieg verantwortlich gemacht werden, beziehen sich nicht direkt auf die Freiwilligentätigkeit. Es handelt sich um berufliche oder familiäre Gründe, um Ortswechsel oder um Altersgründe (vgl. Abbildung 5.2). Die Beendigung des Engagements hat also eher mit der

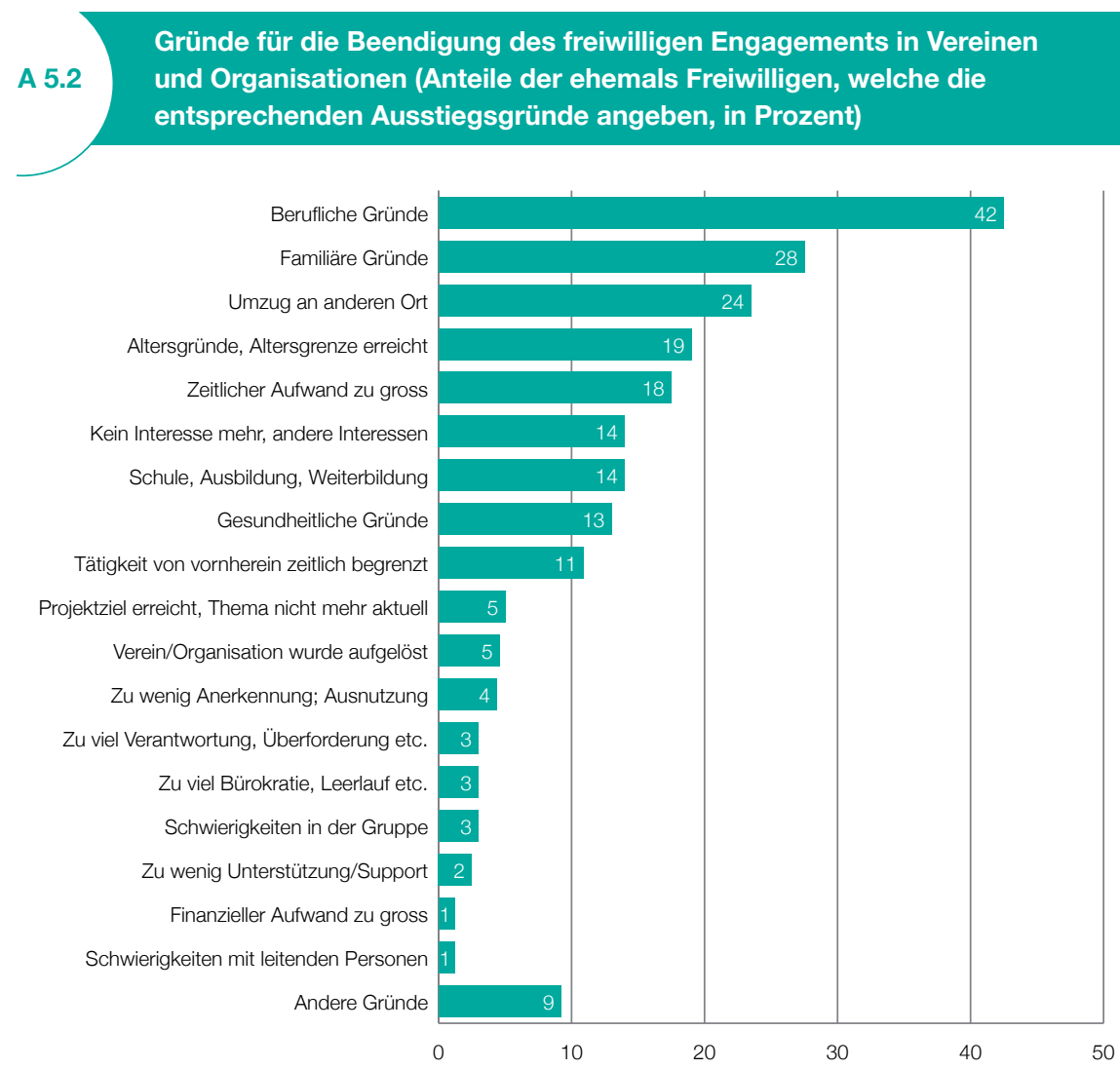

Anzahl Befragte: 1528. 
Belastung und der Work-Life-Balance sowie mit Lebensereignissen zu tun als mit Ärger im Verein oder Unzufriedenheit mit der Organisation. Die Schwierigkeit, Arbeit, Privatleben und Ehrenamt in Einklang zu bringen und der zeitliche Aufwand sind die häufigsten Ausstiegsgründe. Auslöser wie «zu wenig Anerkennung», «Überforderung», «zu viel Verantwortung», «Bürokratie und Leerlauf», «Schwierigkeiten in der Gruppe» sowie «mangelnde Unterstützung» oder «finanzieller Aufwand» kommen zwar vor, werden aber eher selten als Grund für den Ausstieg genannt.

\subsection{Motive der informell Freiwilligen und der Spendenden}

\section{Bei der informellen Freiwilligenarbeit geht es zunächst ums Helfen}

In Abbildung 5.3 sind die Motive aufgeführt, die für das informelle Freiwilligenengagement genannt werden. Hier steht nicht der Spass an erster Stelle, sondern das Helfen. Es liegt in der Natur der Sache, dass bei der informellen Freiwilligenarbeit, bei der Betreuung und Pflege eine zentrale Rolle spielt, die Hilfeleistung stärker betont wird. Dazu gehört auch die häufige Nennung der Motive «anderen Personen etwas zurückgeben» und «das Gefühl, gebraucht zu werden». Beim informellen Engagement schwingen noch stärker als beim formellen Engagement Vorstellungen des sozialen Tauschs und der Reziprozität mit: Leistungen werden zwischen den Generationen zeitverschoben erwidert (vgl. Bourdieu 1998, Nadai 2004).

\section{Auch informelle Freiwilligentätigkeiten machen Spass}

Auch wenn es bei der informellen Freiwilligentätigkeit mehr um die Unterstützung und den Beistand geht, bleibt der Spass nicht einfach auf der Strecke. Gut die Hälfte der informellen Freiwilligen sagt, dass ihnen die Tätigkeit Spass mache. Die finanzielle Entschädigung spielt praktisch keine Rolle, und 


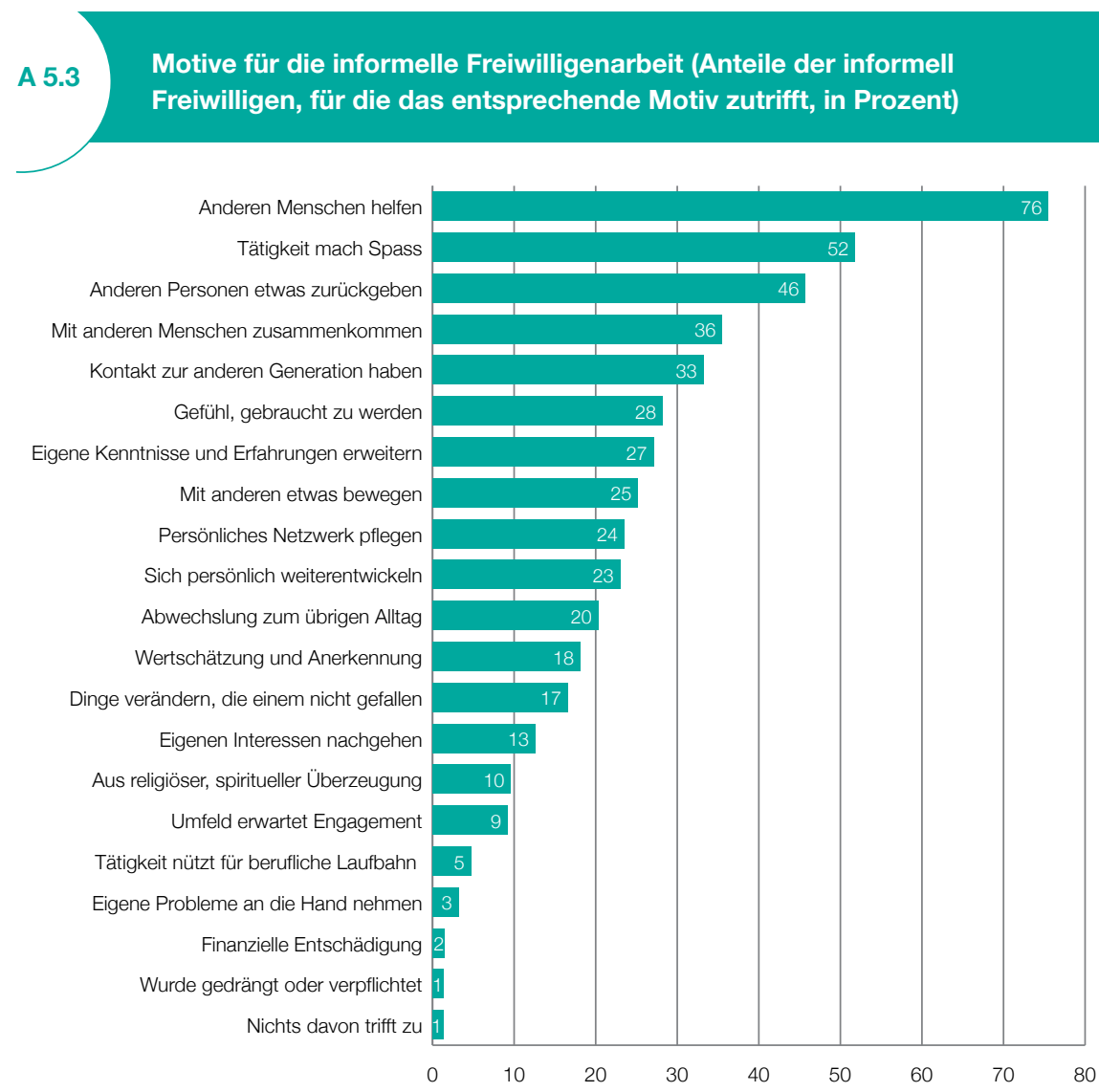

Anzahl Befragte: 2262.

es wird auch kaum jemand explizit gedrängt oder verpflichtet, Erwartungen aus dem Umfeld gibt es dagegen schon. Auch die sozialen Kontakte und die Möglichkeiten, mit anderen etwas bewegen oder sich weiterentwickeln zu können, spielen eine Rolle. Sie sind aber deutlich weniger starke Triebfedern als beim formellen Engagement (vgl. Kapitel 5.1).

Je nach Alter, Geschlecht und Bereich, in dem man sich informell freiwillig engagiert, werden die einzelnen Motive etwas anders gewichtet, ohne dass sich jedoch die Reihenfolge der Motive gross ändert. Während bei den unter 45-Jährigen das Hilfemotiv, das Zusammenkommen mit anderen Menschen, die Pflege des persönlichen Netzwerks sowie die Möglichkeiten, 
mit anderen etwas bewegen oder den eigenen Interessen nachgehen zu können, etwas stärker gewichtet werden, haben bei den über 65-Jährigen der Kontakt zur anderen Generation sowie das Gefühl, gebraucht zu werden, einen etwas höheren Stellenwert. Für Frauen sind das Hilfemotiv, das Zusammenkommen mit anderen, das Gefühl, gebraucht zu werden, sowie die Möglichkeiten, sich persönlich weiterentwickeln und Kenntnisse erweitern zu können, etwas wichtiger als für Männer, und die Tätigkeit bereitet ihnen auch etwas häufiger Spass. Anderen Personen etwas zurückgeben zu können, hat bei der Pflege von Betagten und Kranken einen höheren Stellenwert, während bei der Mithilfe bei gemeinnützigen Projekten öfters die Möglichkeit, mit anderen etwas bewegen und Dinge verändern zu können, wichtig ist. Hier macht die Tätigkeit - ähnlich wie bei der Betreuung von Kindern - auch besonders häufig Spass. Vergleicht man die Motive der informell Freiwilligen im eigentlichen Sinne mit den informell Freiwilligen, von deren Engagement ausschliesslich verwandte Personen profitieren, so werden bei ersteren das Hilfemotiv, das Zusammenkommen mit anderen Menschen, die Gestaltungsmöglichkeiten (gemeinsam etwas bewegen können), die persönlichen Interessen und Entwicklungsmöglichkeiten sowie die Erweiterung von Kenntnissen und Erfahrungen häufiger genannt, während Letztere etwas häufiger den Kontakt zur anderen Generation, die Erwartungen aus dem Umfeld sowie die erhaltene Wertschätzung und Anerkennung erwähnen.

\section{Mit Spenden will man unterstützen, helfen und zum Gemeinwohl beitragen}

Spenden tut man, weil man die Ziele einer Organisation unterstützen, Menschen in Notlagen helfen oder etwas zum Gemeinwohl beitragen will (vgl. Abbildung 5.4). Ein Drittel spendet aber auch, um gewisse Dienstleistungen beziehen zu können, wie man sie zum Beispiel bei einer Mitgliedschaft bei der Rega erhält. Wenn der Bezug der Dienstleistung zum primären Ziel einer Spende wird, könnte man Spenden allerdings nicht mehr als freiwilliges Engagement bezeichnen. Das gleiche gilt, wenn man nur zum Steuersparen spenden würde. Dass man die Spende von den Steuern abziehen kann, scheint allerdings nur ein guter Nebeneffekt, nicht aber ein Hauptmotiv zu sein. Nur ein Achtel der Spendenden gibt den Steuerabzug als Beweggrund an. 
Bei höheren Spendensummen werden nicht nur die Steuerabzugsmöglichkeiten häufiger genannt, sondern auch der Beitrag zum Gemeinwohl, die Hilfe für Menschen in Notsituationen sowie religiöse oder spirituelle Überzeugungen.

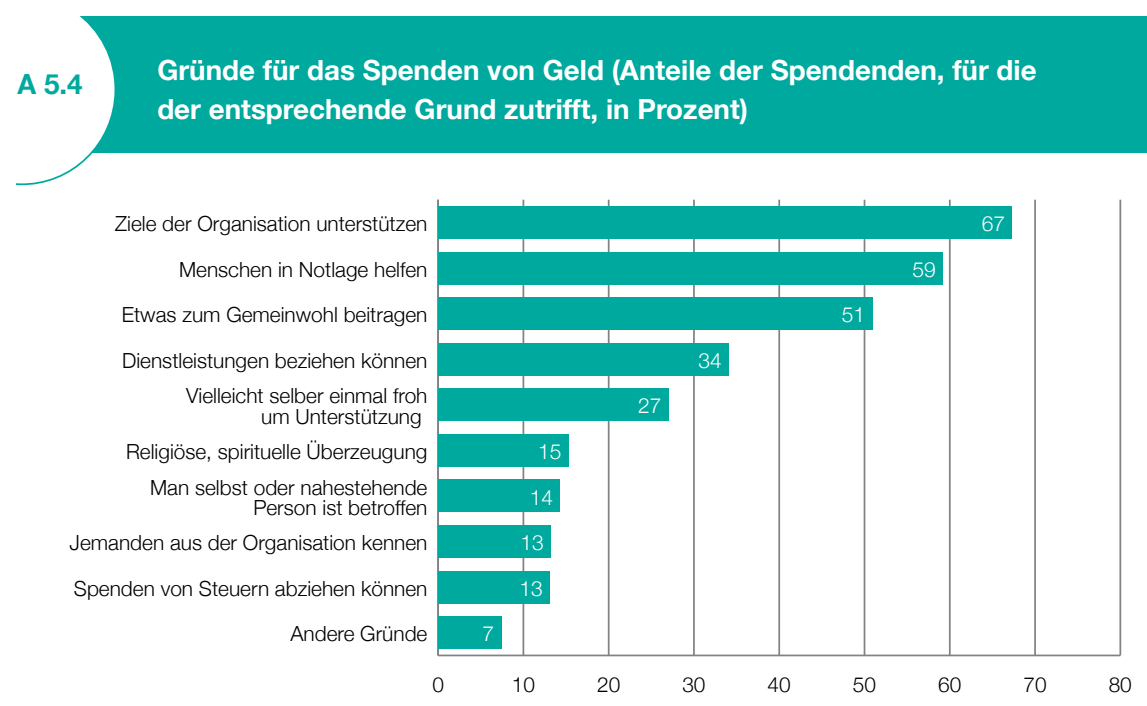

Anzahl Befragte: 3491.

\subsection{Potenzial und Mobilisierung zukünftiger Freiwilliger}

\section{An der grundsätzlichen Bereitschaft fehlt es nicht}

Im Folgenden wollen wir uns dem Potenzial der Freiwilligenarbeit zuwenden und der Frage, wie dieses Potenzial besser genutzt werden kann. Dazu legen wir zunächst den Fokus auf die 61 Prozent der Schweizer Bevölkerung, die sich nicht in einem Verein oder einer Organisation freiwillig engagieren. Diese Personen, die keine formelle Freiwilligenarbeit leisten, wurden gefragt, ob sie interessiert wären, sich (wieder) in Vereinen und Organisationen zu 
engagieren und dort freiwillig und unentgeltlich Aufgaben zu übernehmen. 16 Prozent beantworten die Frage mit «ja, sicher», 50 Prozent mit «ja, vielleicht» und nur 34 Prozent mit «nein» (vgl. Abbildung 5.5). Selbst wenn man einräumt, dass diese Frage völlig unverbindlich ist, und die Personen, die im Monitor Auskunft geben, ein überdurchschnittliches Interesse an Freiwilligkeit haben, offenbaren diese Zahlen ein grosses Potenzial für formelle Freiwilligenarbeit. Umgerechnet auf die Bevölkerung könnten sich 4o Prozent der in der Schweiz lebenden Personen vorstellen, (wieder) einmal formelle Freiwilligenarbeit zu leisten.

\section{Das ungenutzte Potenzial}

Bei diesen potenziellen formell Freiwilligen handelt es sich häufiger um Personen, die bereits Mitglied in einem Verein oder einer Organisation sind (vgl. Abbildung 5.5). Es ist aber bemerkenswert, dass sich auch viele Nichtmitglieder vorstellen könnten, zukünftig Aufgaben oder Arbeiten in einem Verein oder einer Organisation zu übernehmen. Für Vereine und Organisationen bedeutet dies, dass es sich bei der Suche nach Freiwilligen lohnen würde, nicht nur unter den eigenen Mitgliedern Ausschau zu halten.

Aufgrund der Befunde in Abschnitt 5.2 ist es wenig erstaunlich, dass Personen, die schon einmal in einem Verein oder einer Organisation freiwillig tätig waren, häufiger mit einem klaren «ja» auf die Fragen nach einem möglichen Wiedereinstieg antworten. Eine höhere Bereitschaft signalisieren auch Frauen, jüngere Personen, Personen aus der französisch- und italienischsprachigen Schweiz, StadtbewohnerInnen und in der Schweiz lebende Ausländerinnen und Ausländer. Ein überdurchschnittliches Interesse zeigen also genau jene Bevölkerungsgruppen, die heute noch weniger in die formelle Freiwilligenarbeit einbezogen sind (man vergleiche dazu Abbildung 5.5 mit Abbildung 2.3.).

$\mathrm{Zu}$ den Personen, die sich noch nicht freiwillig engagieren, kommen noch diejenigen, die bereits formelle Freiwilligenarbeit leisten und sich vorstellen können, ihr Engagement auszubauen. Von den Freiwilligen in Vereinen und Organisationen können sich 16 Prozent vorstellen, ihr formelles Engagement auszubauen. Weitere 35 Prozent antworten auf die Fragen nach einem Ausbau des Engagements mit «eher ja», während 44 Prozent «eher nein» 

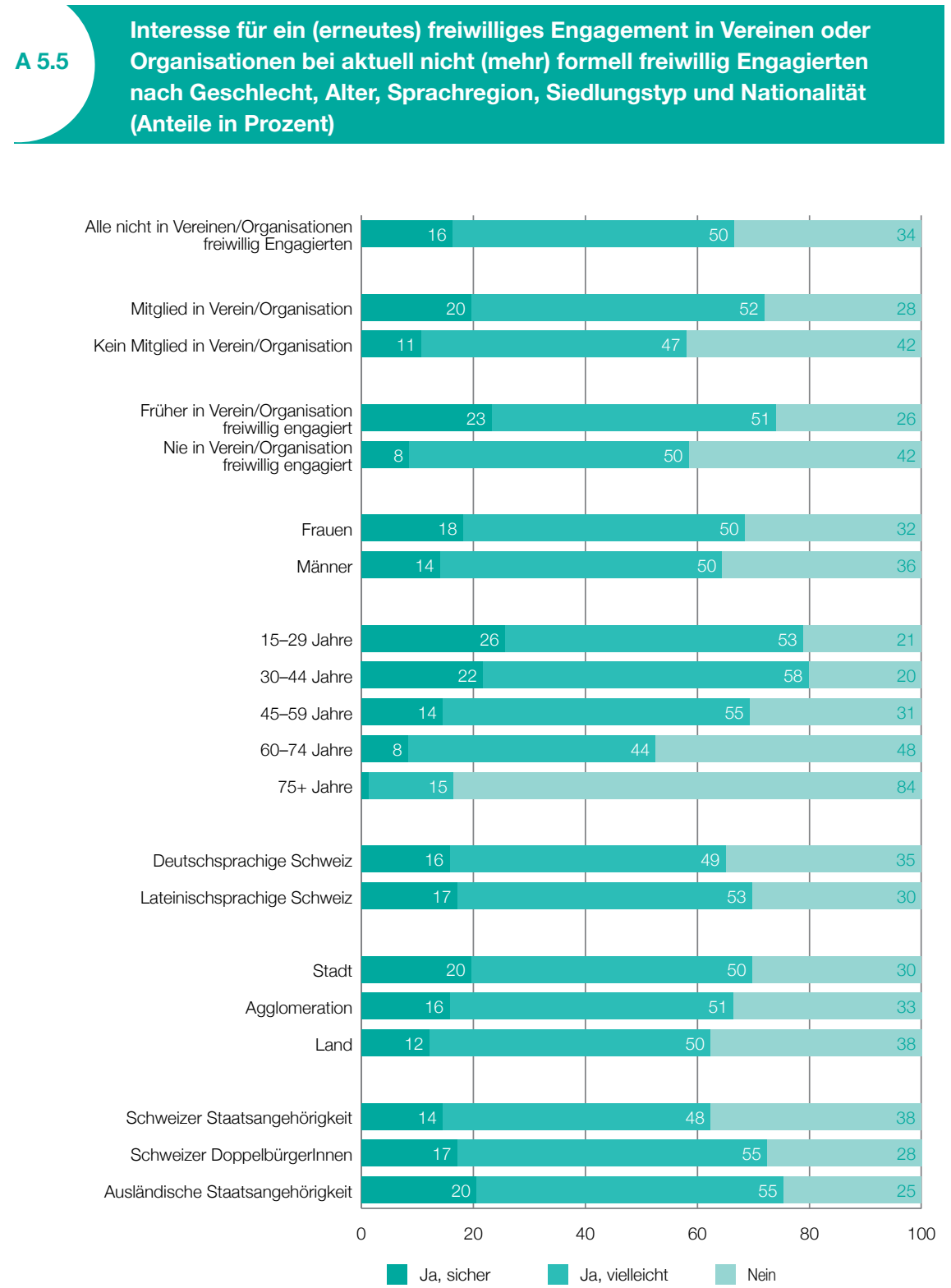

Anzahl Befragte: 2956. Lesebeispiel: Von den aktuell nicht formell freiwillig Engagierten, die sich aber früher einmal in dieser Form engagierten, sind 23 Prozent sicher interessiert, sich wieder in Vereinen oder Organisationen freiwillig zu engagieren, 51 Prozent sind vielleicht interessiert und 26 Prozent sind nicht interessiert. 
und 5 Prozent «nein, auf keinen Fall» angekreuzt haben. Freiwillige, die sich vorstellen können ihr Engagement auszubauen, sind jünger, eher weiblich, eher Personen aus der Romandie oder dem Tessin, eher StädterInnen und eher Ausländerinnen und Ausländer. Das Profil entspricht also genau jenem der potenziellen WiedereinsteigerInnen und unterstreicht das Potenzial in den Bevölkerungsgruppen, die heute noch weniger in die formelle Freiwilligenarbeit eingebunden sind.

\section{Bedingungen für Einstieg: Zeit, gutes Thema, Flexibilität, ein tolles Team}

Von einer einfachen Interessensbekundung in einem Fragebogen bis zur tatsächlichen Übernahme einer Funktion in einem Verein oder einer Organisation kann es ein langer Weg sein. Fragt man die Leute, was passieren müsste, dass sie diesen Schritt tatsächlich wagen würden, erhält man die in Abbildung 5.6 aufgeführten Antworten. Als erste Bedingung müssten die an Freiwilligenarbeit interessierten Personen genügend Zeit haben. Dann müsste es ein gutes Thema bzw. Anliegen sein, das Engagement dürfte nicht an allzu fixe Zeiten gebunden sein und es müssten die richtigen Leute mitmachen. Wichtig wäre auch, dass man konkret angefragt wird oder über Einsatzmöglichkeiten informiert ist und dass sich der Einsatz zeitlich begrenzen lässt. Die Anerkennung und Wertschätzung der Arbeit sowie die fachliche Unterstützung werden von einem Zehntel als Voraussetzung für ein Engagement genannt.

\section{Wenig Zeit, mangelndes Interesse, Angst vor Verpflichtungen sowie Alter verhindern Engagement}

Fragt man umgekehrt jene Personen, die kein Interesse an der Übernahme von Aufgaben und Arbeiten in einem Verein oder einer Organisation haben, weshalb sie sich nicht engagieren wollen, so wird zunächst ebenfalls auf die fehlende Zeit verwiesen: 39 Prozent geben an, keine Zeit dafür zu haben. Weitere Gründe sind: «kein Interesse, andere Interessen/Verpflichtungen» (32\%), «möchte mich nicht für regelmässiges Engagement verpflichten» (31\%), «bin zu alt» $(31 \%)$ und «gesundheitliche Gründe bzw. zu wenig Energie» (13\%). 
Nur wenige Nennungen (je unter $5 \%$ ) erhalten dagegen: «zu wenig Anerkennung», «zu wenig qualifiziert», «zu wenige Vorteile und Vergünstigungen», «zu jung» und «keine Ansprechpartner».

\section{Freiwillige müssen persönlich angefragt und überzeugt werden}

Aus Abbildung 5.6 wird deutlich, dass ein Fünftel der potenziellen Freiwilligen konkret für ein Engagement angefragt werden müsste. Wie wichtig für die Gewinnung der Freiwilligen der persönliche Kontakt und die effektive Anfrage sind, zeigt sich auch, wenn wir die zur Zeit aktiven formell Freiwilligen fragen, woher für sie der Anstoss kam, ihre Freiwilligentätigkeit zu übernehmen. 46 Prozent der Freiwilligen wurden von (leitenden) Personen aus den jeweiligen Vereinen und Organisationen überzeugt und bewegt. Für 31 Prozent kam der Anstoss von FreundInnen und Bekannten, für 15 Prozent von Familienmitgliedern, für 8 Prozent von den eigenen Kindern, die im Verein aktiv waren, für 7 Prozent von der Gemeinde und für 3 Prozent vom

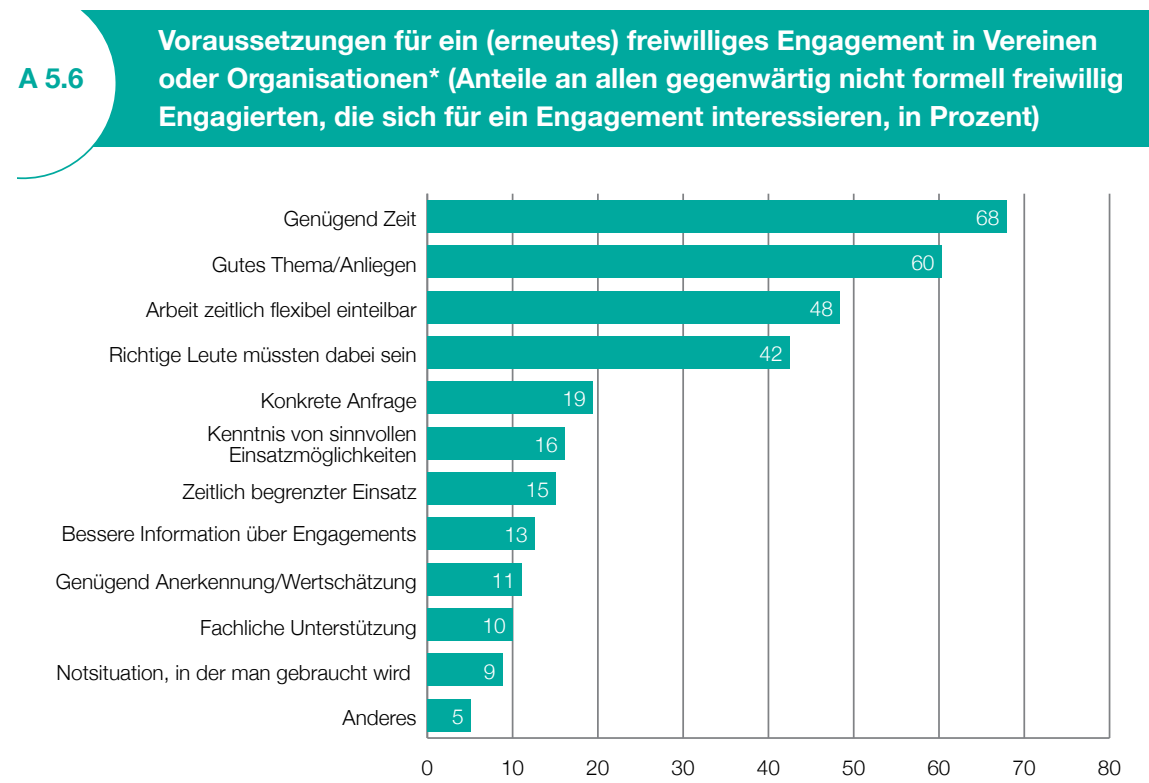

Anzahl Befragte: 1945. Hinweis: * Antwort auf die Frage: «Was müsste passieren, damit Sie sich in Vereinen oder Organisationen freiwillig engagieren?» 
Arbeitgeber. Relativ selten - d.h. von jeweils höchstens 3 Prozent - werden Informations- oder Kontaktstellen, Internet-Plattformen und soziale Netzwerke, Hinweise aus Presse, Radio und Fernsehen oder spezielle Internetplattformen genannt. Allerdings muss man hier berücksichtigen, dass die Übernahme der Funktion häufig schon weit über zehn Jahre zurückliegt und die neuen Möglichkeiten, die sich heute bieten, noch nicht genutzt werden konnten. Das direkte Ansprechen und Überzeugen dürfte aber auch zukünftig der Königsweg zur Freiwilligenarbeit bleiben. Wenn die zukünftigen Freiwilligen zudem motiviert sind, Spass haben und Kompetenzen entwickeln können, fällt die Überzeugungsarbeit sicher leichter. Immerhin 37 Prozent der heutigen Freiwilligen sagen nämlich, es sei ihnen ein Bedürfnis gewesen sich zu engagieren. Bei 23 Prozent kam der Anstoss von eigenen Erlebnissen und Erfahrungen und 17 Prozent sagen, sie seien in die Freiwilligenarbeit hineingewachsen.

\section{Soziale Organisationen und Umweltorganisationen als Wunschbereiche}

Die Hälfte der Personen, die sich für ein erneutes oder erstmaliges freiwilliges Engagement in Vereinen oder Organisationen interessieren, hat schon konkrete Vorstellungen, wo sie sich engagieren möchten. Tabelle 5.1 gibt einen Überblick über die Wunschbereiche der potenziellen Freiwilligen. Bemerkenswert sind die häufigen Nennungen von sozialen und karitativen Organisationen sowie der Umwelt- und Tierschutzorganisationen. Letztere werden besonders häufig auch von Personen genannt, die sich bis anhin nicht formell freiwillig engagiert haben. Die Umwelt- und Tierschutzorganisationen sind auch der Bereich mit der grössten Differenz zwischen aktuellem und zukünftig denkbarem Engagement. Das aktuelle Engagement in diesem Bereich ist noch relativ tief, das Interesse für ein zukünftiges Engagement aber umso grösser. Genau umgekehrt ist es beim Sportverein: Hier engagieren sich aktuell am meisten Freiwillige; das Interesse für ein zukünftiges Engagement ist aber deutlich geringer als bei den sozialen Organisationen und den Umwelt- und Tierschutzorganisationen. 


\begin{tabular}{|c|c|c|}
\hline & $\begin{array}{l}\text { Personen mit früherem } \\
\text { Engagement }\end{array}$ & $\begin{array}{c}\text { Personen ohne früheres } \\
\text { Engagement }\end{array}$ \\
\hline Soziale, karitative, gemeinnützige Organisation & 43 & 40 \\
\hline Umwelt-, Tierschutzorganisation & 34 & 48 \\
\hline Sportclub, -verein & 32 & 24 \\
\hline Kultureller Verein & 32 & 15 \\
\hline Spiel-, Hobby-, Freizeitverein & 23 & 22 \\
\hline Menschenrechtsorganisation & 19 & 25 \\
\hline Gemeinde-, Orts-, Quartierverein & 20 & 14 \\
\hline Selbsthilfegruppe & 10 & 16 \\
\hline Elternrat, Elternvertretung an Schulen & 8 & 10 \\
\hline Öffentlicher Dienst & 7 & 11 \\
\hline Politisches oder öffentliches Gremium/Amt & 10 & 6 \\
\hline Religionsgemeinschaft, Kirche oder kirchennahe Organisation & 10 & 5 \\
\hline Interessenverband & 9 & 6 \\
\hline Jugendorganisation & 8 & 6 \\
\hline Politische Partei & 8 & 4 \\
\hline
\end{tabular}

Anzahl Befragte: Personen mit früherem freiwilligen Engagement in Vereinen oder Organisationen: 604; Personen ohne früheres freiwilliges Engagement in Vereinen oder Organisationen: 365. Lesebeispiel: 32 Prozent der Personen, die sich früher formell freiwillig engagiert haben und interessiert wären, sich wieder freiwillig zu engagieren - und auch schon eine Vorstellung haben, in welchem Bereich das wäre - nennen einen kulturellen Verein als Wunschbereich. Bei den Personen ohne ein früheres Engagement sind es 15 Prozent.

\subsection{Unterstützungsleistungen für die Freiwilligenarbeit}

Alle Personen, die formelle Freiwilligenarbeit leisten, wurden nach Massnahmen gefragt, mit denen man das ehrenamtliche und freiwillige Engagement fördern und unterstützen könnte. Die Befragten sollten dabei ganz konkret an Verbesserungen ihrer eigenen Situation denken. In der oberen Hälfte von Abbildung 5.7 geht es um Veränderungen durch den Verein oder die Organisation selbst, in der unteren Hälfte um Unterstützungsleistungen seitens der Öffentlichkeit, der Politik und des Arbeitsgebers. Letztere werden häufiger gewählt als erstere: ${ }^{1}$ Prozent der Freiwilligen haben keine entsprechenden Verbesserungsvorschläge an ihre Vereine und Organisationen, 24 Prozent 

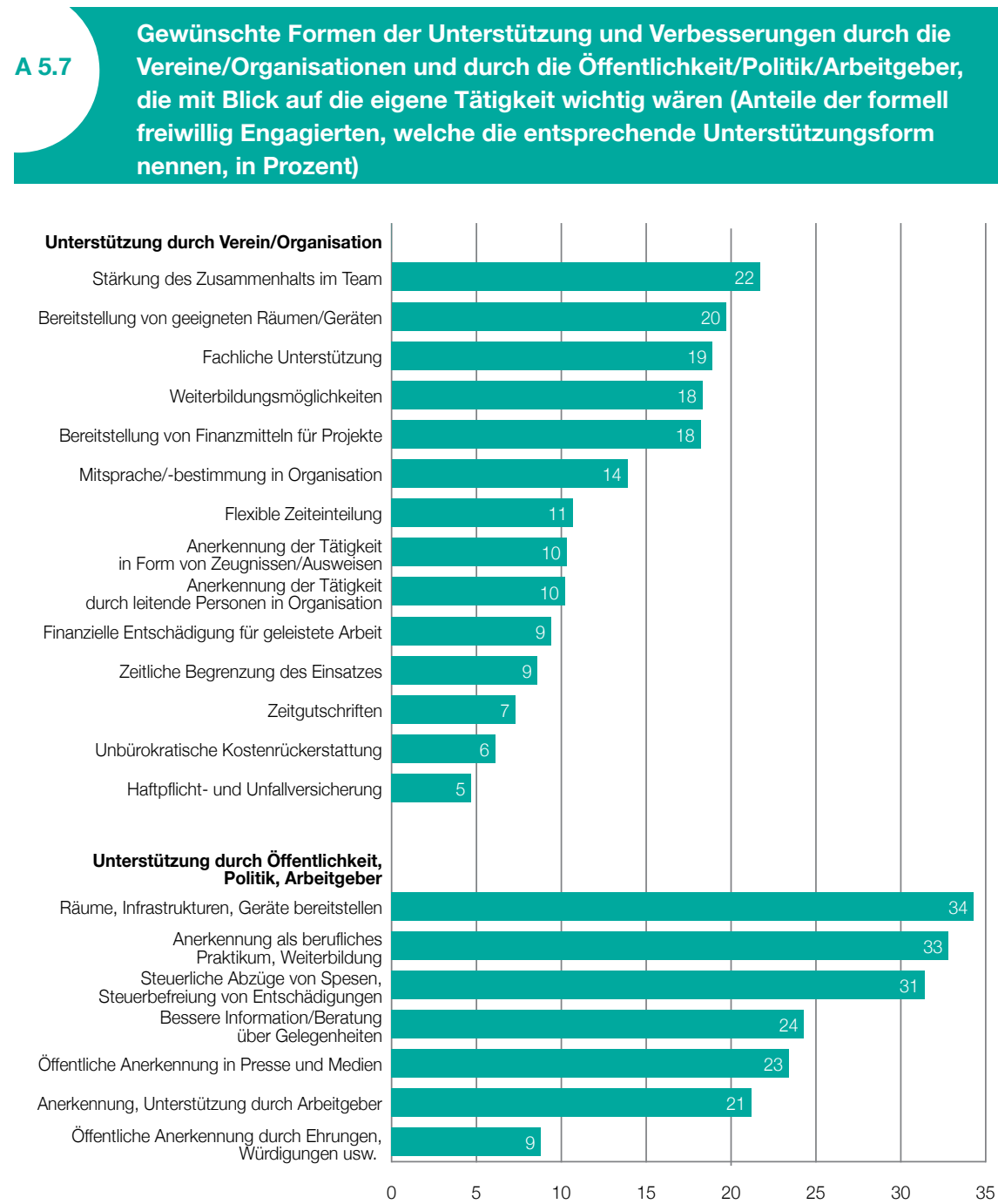

Anzahl Befragte: Unterstützung durch Verein/Organisation: 1908; Unterstützung durch Öffentlichkeit, Politik, Arbeitgeber: 1895.

brauchen keine zusätzlichen Unterstützungsleistungen durch Öffentlichkeit, Politik und Arbeitgeber.

Die Ehrenamtlichen sehen bei fast allen Punkten mehr Verbesserungspotenzial als die Freiwilligen ohne gewähltes Amt. Dies dürfte auch daran liegen, dass sich Ehrenamtliche mehr mit ihrer Aufgabe identifizieren, 
mehr Verantwortung tragen und besser hinter die Kulissen der formellen Freiwilligenarbeit sehen.

\section{Breite Palette an Verbesserungsvorschlägen für Vereine und Organisationen}

Die Freiwilligen wünschen sich mit Blick auf ihre Tätigkeit die folgenden Verbesserungen durch ihren Verein oder ihre Organisation (vgl. Abbildung 5.7): Verbesserungen bei der Teambildung und der Infrastruktur, fachliche Unterstützung, Weiterbildungsmöglichkeiten und mehr Finanzmittel für bestimmte Projekte. Daneben wird eine ganze Palette weiterer Massnahmen genannt: von mehr Mitsprache, flexiblerer Zeiteinteilung, mehr Anerkennung (durch Zeugnisse und Ausweise sowie seitens der hauptamtlichen Personen) über höhere finanzielle Entschädigungen, zeitliche Begrenzung der Einsätze bis zu Zeitgutschriften, Kostenrückerstattung oder Versicherungsleistungen. Auffällig ist, dass es nicht eine Fördermassnahme gibt, die klar heraussticht, sondern einen ganzen Strauss von möglichen Massnahmen. In den Antworten widerspiegeln sich die unterschiedlichen Situationen in den Vereinen und Organisationen und die Heterogenität der Herausforderungen und Probleme. Es lassen sich auch nicht einfache Zuordnungen einzelner Problemlagen und Verbesserungsvorschläge für die jeweiligen Bereiche finden, sondern nur leicht unterschiedliche Gewichtungen. Freiwillige in politischen Parteien, öffentlichen Ämtern und Interessenverbänden wünschen sich etwas häufiger mehr fachliche Unterstützung. Finanzielle Mittel für Projekte wären noch etwas stärker in Menschenrechts- und Umweltverbänden, in politischen Parteien und in Jugendorganisationen gefragt, und die Bereitstellung geeigneter Räume und Geräte ist ein Anliegen, das von den Freiwilligen in Religionsgemeinschaften und in Spiel-, Hobby- und Freizeitvereinen etwas häufiger genannt wird.

\section{Unterstützungsmassnahmen durch Öffentlichkeit, Politik und Arbeitgeber}

Mit Blick auf mögliche Unterstützungsmassnahmen durch die Öffentlichkeit, die Politik oder die Arbeitgeber stehen die Bereitstellung von Infrastrukturen 
und Geräten, die Anerkennung der Tätigkeit als berufliches Praktikum oder Weiterbildung sowie die Steuerbefreiung von Entschädigungen ganz oben auf der Wunschliste. Verbesserungspotenzial sieht man auch bei der Information über Einsatzmöglichkeiten sowie bei der Anerkennung durch die Medien und durch den Arbeitgeber. Der öffentlichen Anerkennung durch Ehrungen und Würdigungen kommt dagegen eine geringere Bedeutung zu. Auch hier lassen sich nicht bestimmte Forderungen einzelnen Bereichen zuordnen, sondern es zeigen sich eher graduelle Unterschiede. Die Anerkennung der Tätigkeit als berufliches Praktikum wird beispielsweise in Menschenrechtsund Umweltverbänden etwas stärker thematisiert, eine bessere Information und Beratung zu freiwilligen Tätigkeiten hingegen in sozialen und karitativen Organisationen.

\section{Unterstützung der Betreuungs- und Pflegearbeit durch den Staat}

Auch die informell Freiwilligen, welche sich um Betagte, Kranke oder Behinderte kümmern, wurden gefragt, ob sie sich bei der Betreuung und Pflege dieser Personen mehr Unterstützung wünschen. Während 58 Prozent keine Unterstützung brauchen, würden 12 Prozent mehr Unterstützung klar begrüssen und zo Prozent zumindest teilweise. Bei denjenigen, die sich mehr Unterstützung wünschen, richtet sich der Wunsch am häufigsten an den Staat bzw. die Gemeinde (58\%), zum Beispiel mit Leistungen in Form von Spitexangeboten, Kinderkrippen oder Betreuungsgutschriften. 37 Prozent wünschen sich mehr Unterstützung durch Angehörige oder Verwandte, 15 Prozent vom Arbeitgeber (z. B. durch flexible Arbeitszeiten) und 22 Prozent von anderen Stellen.

\section{Verantwortung von Angehörigen, Umfeld, Staat, Wirtschaft und Freiwilligenorganisationen}

Sämtliche Personen - unabhängig davon, ob sie sich engagieren oder nicht wurden gefragt, wer ihrer Ansicht nach mehr Verantwortung und Aufgaben übernehmen sollte, wenn einzelne Personen und Familien überfordert sind und Hilfe benötigen. Häufig sehen die Befragten mehrere AkteurInnen in der 
Pflicht (vgl. Abbildung 5.8). Zwei Drittel erwarten ein höheres Engagement von Angehörigen oder Personen aus dem jeweiligen sozialen Umfeld, knapp 60 Prozent von staatlichen Stellen. Auch von Freiwilligenorganisationen und von der Wirtschaft wird ein Beitrag erwartet.
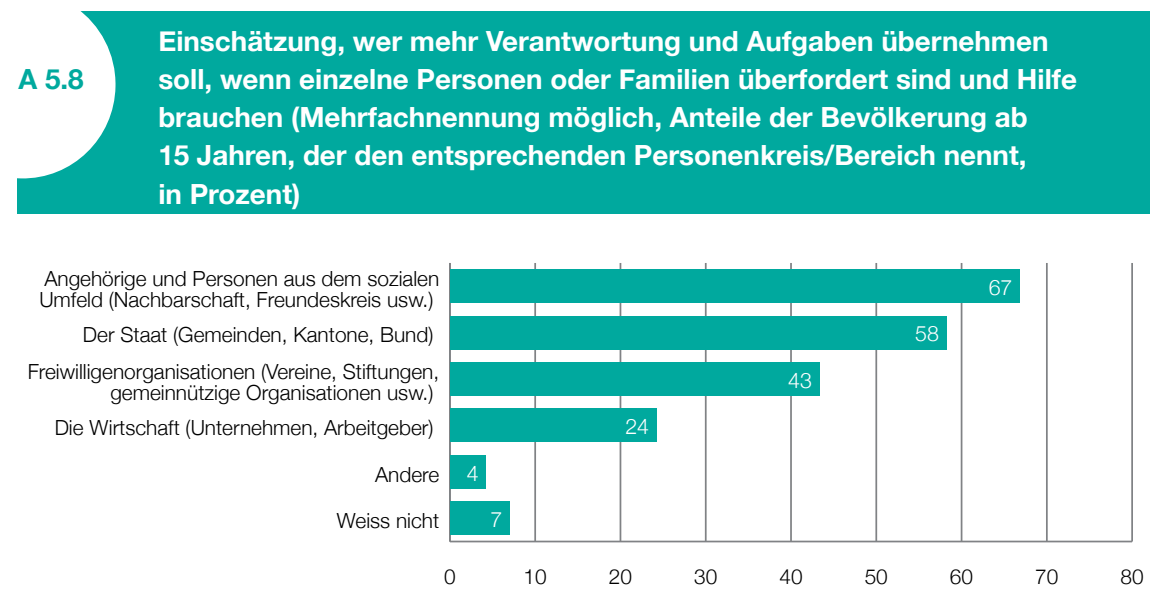

Anzahl Befragte: 4907.

Frauen messen den Freiwilligenorganisationen ein etwas stärkeres Gewicht bei als Männer, und in der Deutschschweiz sind die Erwartungen gegenüber den Angehörigen und dem sozialen Umfeld noch etwas höher (69\%) als in der Romandie (6o \%) und im Tessin (62 \%). Auch bei Personen, die tatsächlich Betagte, Kranke oder Behinderte betreuen und pflegen, rangieren die Angehörigen und das soziale Umfeld sowie der Staat an erster Stelle, sie betonen aber auch die Bedeutung der Freiwilligenorganisationen (von 52 \% genannt).

\section{Beachtliche Unterstützungsleistungen durch die Arbeitgeber}

Wer sich formell oder informell freiwillig engagiert und gleichzeitig erwerbstätig und angestellt ist, wurde gefragt, ob sie oder er vom Arbeitgeber bei der Freiwilligenarbeit unterstützt wird. Bei 37 Prozent ist dies der Fall, bei 63 Prozent nicht. Männer profitieren häufiger von einer Unterstützung 
ihres Arbeitgebers (41\%) als Frauen (34\%). Personen mit einem Engagement in Vereinen oder Organisationen werden eher unterstützt (41\%) als Personen, die sich informell freiwillig engagieren (31\%). Auch wenn man nur die formell Freiwilligen betrachtet, bleibt ein Geschlechterunterschied bestehen. In der Deutschschweiz können die Freiwilligen eher auf die Unterstützung ihres Arbeitgebers zählen als in der Romandie.

Die häufigste Form der Unterstützung sind flexible Arbeitszeiten (65\%). 34 Prozent können bestimmte Infrastrukturen (Räume, Telefon, Kopierer etc.) nutzen, 31 Prozent werden zumindest teilweise für ihre Freiwilligentätigkeiten freigestellt, 22 Prozent geniessen unterschiedliche Formen der Anerkennung (von Lob bis hin zur Beförderung) und 12 Prozent nennen andere Formen der Unterstützung. 


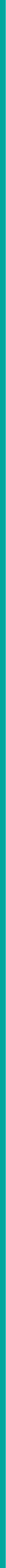


1 Freiwilligkeit

2 Formelle Freiwilligenarbeit und Ehrenamt

3 Informelle Freiwilligenarbeit

4 Spenden und weitere Formen von Freiwilligkeit

5 Motive, Mobilisierung und Potenzial

6 Politisches Engagement, Vertrauen und die Freiwilligkeit

Untersuchungsmethode und Stichprobe

Literaturverzeichnis 

Dass freiwilliges Engagement nicht nur zur Integration und zum Zusammenhalt der Gesellschaft beiträgt, sondern auch Vertrauen schaffen kann, ist wissenschaftlich hinreichend belegt (vgl. Freitag 2016; Putnam 2000; Traunmüller et al. 2012; Lamprecht et al. 2018). In ihrem Buch zum sozialen Kapital in der Schweiz bezeichnen Freitag und Bauer (2016) Freiwilligenorganisationen in Anlehnung an de Tocqueville (2016 [1835]) nicht nur als «Schulen der Demokratie», sondern auch als «Schulen des Vertrauens». In diesem Kapitel wird der Zusammenhang von politischem Interesse, politischer Beteiligung, Vertrauen und Freiwilligkeit genauer unter die Lupe genommen.

\section{Interesse an Politik immer noch hoch, aber je nach Bevölkerungsgruppe unterschiedlich}

Auf einer Skala von o (gar nicht an Politik interessiert) bis 1o (sehr stark an Politik interessiert) kommt die Schweizer Bevölkerung auf einen durchschnittlichen Wert von 5.9. Ein hohes Interesse (7-10) haben 47 Prozent, ein mittleres Interesse (4-6) 33 Prozent und 20 Prozent interessieren sich nur wenig oder gar nicht für Politik (o-3). Männer interessieren sich mehr für Politik als Frauen, ältere Personen mehr als jüngere, DeutschschweizerInnen
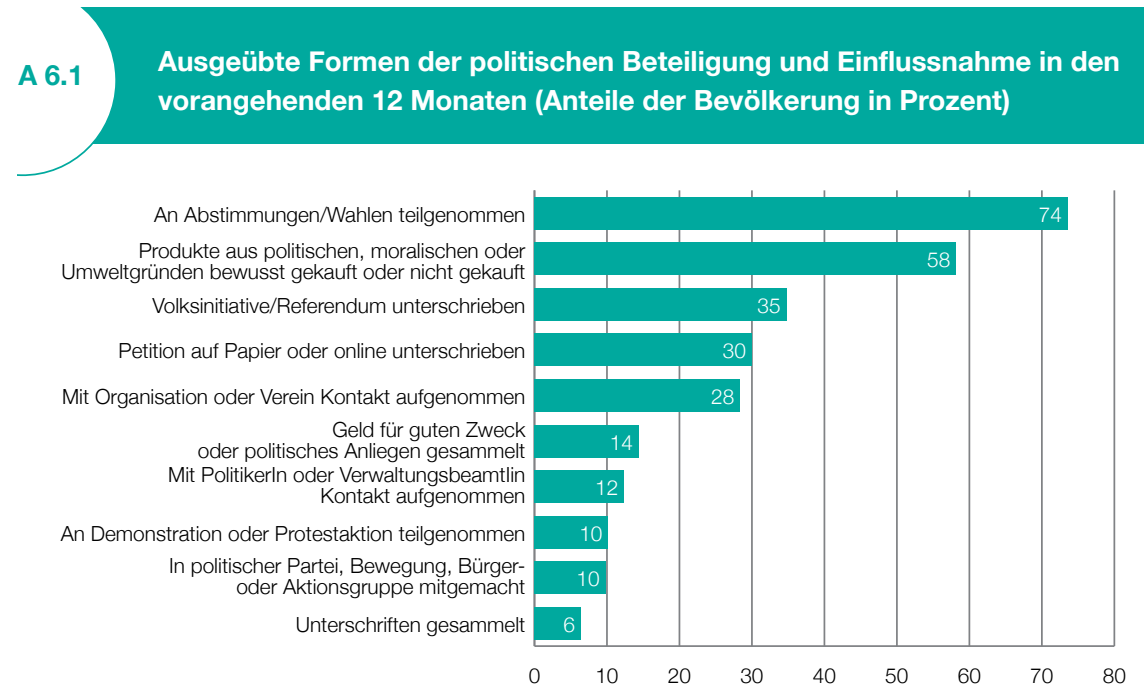

Anzahl Befragte: 4491. 
mehr als die Romands und TessinerInnen, Städter mehr als BewohnerInnen in der Agglomeration und auf dem Land, Schweizer mehr als die in der Schweiz lebenden AusländerInnen. Das Interesse an Politik steigt zudem mit der Bildung und dem Einkommen. Von den verschiedenen Möglichkeiten, wie man sich politisch beteiligen und Einfluss nehmen kann, steht das Abstimmen und Wählen an erster Stelle (vgl. Abbildung 6.1). Relativ häufig werden auch Dinge aus politischen und moralischen Gründen gekauft bzw. nicht gekauft oder Initiativen, Referenden und Petitionen unterschrieben. Die Teilnahme an Protestaktionen oder das Mitmachen in einer Partei, politischen Bewegung oder Bürger- und Aktionsgruppe sind dagegen selten.

\section{Hohes Vertrauen in den sozialen Nahraum, weniger Vertrauen in Fremde und Medien}

Wie das politische Interesse wurde auch das Vertrauen auf einer Skala von o (überhaupt kein Vertrauen) bis 10 (volles Vertrauen) gemessen, wobei unterschieden wurde zwischen Vertrauen gegenüber unterschiedlichen Personengruppen einerseits und verschiedenen politischen und gesellschaftlichen Instanzen andererseits (vgl. Abbildung 6.2). Die Mehrheit der Bevölkerung vertraut ihren Mitmenschen, wobei es beträchtliche Unterschiede gibt, je nachdem wie nahe man jemandem steht. Während das Vertrauen gegenüber Verwandten und FreundInnen sehr gross ist, bleibt man gegenüber fremden Personen deutlich skeptischer. Nur noch 21 Prozent haben ein hohes Vertrauen in Personen, denen sie zum ersten Mal begegnen. Interessant sind die Unterschiede im Vertrauen gegenüber verschiedenen Einrichtungen des öffentlichen Lebens. Das Vertrauen in die Gerichte und die Gemeindeverwaltung, aber auch in den Bundesrat ist signifikant höher als das Vertrauen ins nationale Parlament. Während eine klare Mehrheit ein hohes Vertrauen in die Wissenschaft hat, schlägt den Medien deutlich mehr Misstrauen entgegen. Die Unterschiede nach Geschlecht, Alter, Wohnregion, Nationalität, Bildung und Einkommen sind beim Vertrauen weniger stark als beim politischen Interesse. Zwischen Männern und Frauen gibt es beispielsweise keine signifikanten Unterschiede bezüglich des Vertrauens gegenüber Personen mit der einzigen Ausnahme, dass Frauen ihren Freundinnen und Freunden noch etwas mehr vertrauen als Männer. Zwei markante Unterschiede sind 
dennoch erwähnenswert: Das Vertrauen in die Wissenschaft wächst mit der Höhe des Bildungsabschlusses deutlich an, und die in der Schweiz lebenden Ausländerinnen und Ausländer vertrauen den politischen Institutionen in der Schweiz mehr als die einheimische Bevölkerung.
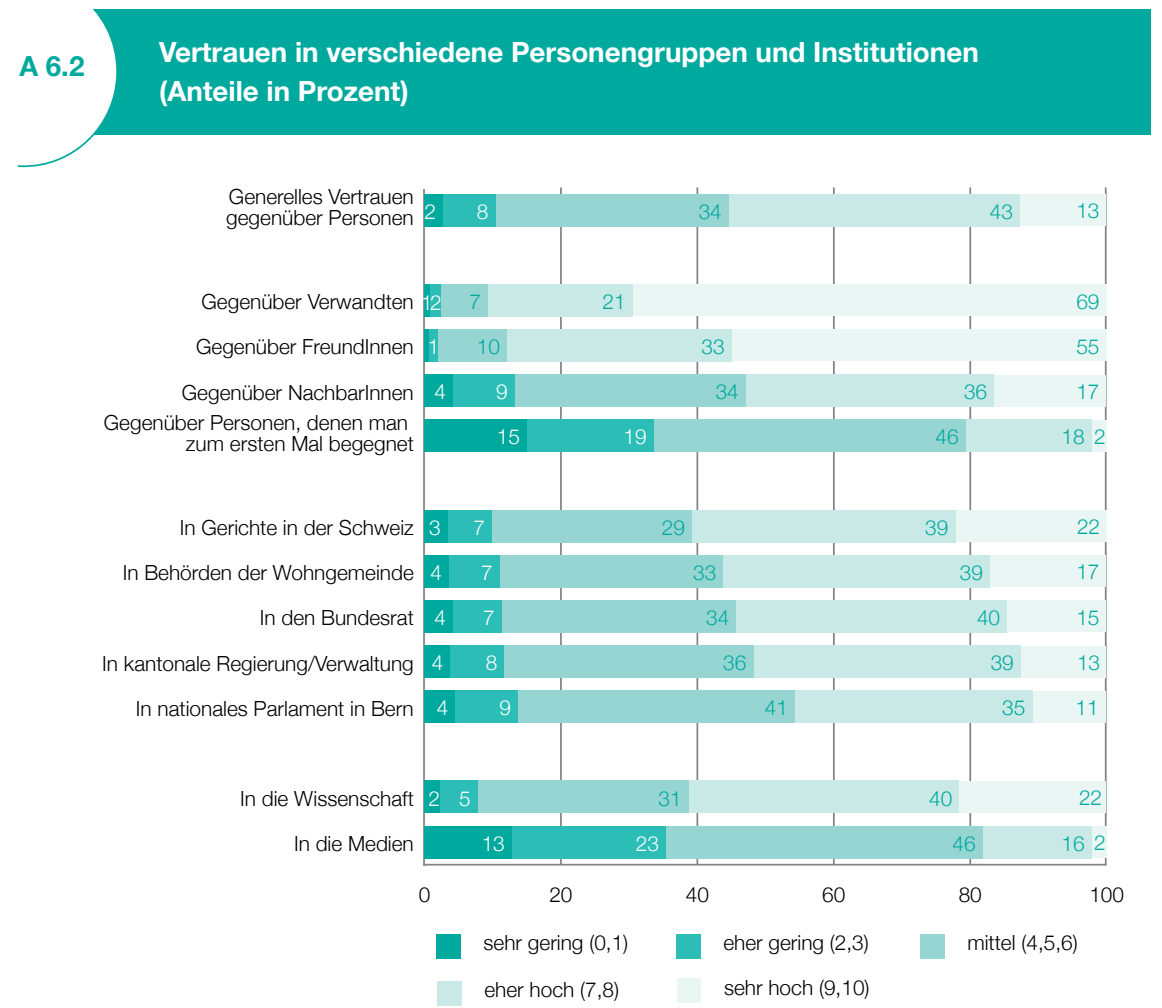

Anzahl Befragte: zwischen 4927 und 4941. Lesebeispiel: Bei der Einstufung des Vertrauens gegenüber den Nachbarlnnen auf einer Skala von 0 bis 10, auf welcher 0 «überhaupt kein Vertrauen» und 10 «volles Vertrauen» bedeutet, äussern 4 Prozent ein sehr geringes Vertrauen (Werte 0 oder 1), 9 Prozent ein eher geringes Vertrauen (Werte 2 oder 3), 34 Prozent ein mittleres Vertrauen (Werte 4 bis 6), 36 Prozent ein eher hohes Vertrauen (Werte 7 oder 8) und 17 Prozent ein sehr hohes Vertrauen (Werte 9 oder 10).

\section{Treffen mit Freunden, Verwandten und Kolleginnen}

In Zusammenhang mit dem Vertrauen gegenüber Personen wurde auch erfragt, wie oft man sich mit Freundinnen und Freunden, mit Verwandten oder Kolleginnen und Kollegen trifft, die nicht im eigenen Haushalt leben. Während genau die Hälfte das mindestens mehrmals pro Woche tut, tref- 
fen sich 1o Prozent der Bevölkerung höchstens einmal im Monat mit ihnen nahestehenden Personen. Grosse Unterschiede gibt es dabei zwischen den Altersgruppen und den Sprachregionen. Je älter man wird, desto weniger trifft man sich mit Freundinnen, Verwandten und Kollegen, und in der Romandie und im Tessin sind Treffen unter FreundInnen und Verwandten deutlich häufiger als in der Deutschschweiz.

\section{Freiwillige haben mehr Vertrauen in ihre Mitmenschen}

Wie Freiwilligkeit mit Vertrauen zusammenhängt, veranschaulicht Abbildung 6.3. Personen, die Freiwilligenarbeit leisten, haben deutlich mehr Vertrauen in ihre Mitmenschen als Personen, die nicht freiwillig tätig sind. Wenn sie nicht nur informelle, sondern auch formelle Freiwilligentätigkeiten übernehmen, steigt das Vertrauen nochmals zusätzlich an. Die Unterschiede im Vertrauen zeigen sich sowohl gegenüber Personen im sozialen Nahraum als auch gegenüber Fremden. Personen, die sich häufig mit Freunden, Verwandten oder Kolleginnen treffen, haben zudem deutlich mehr Vertrauen in ihre Mitmenschen als Personen, die weniger Kontakt zu nahestehenden Personen haben.

Beim Vertrauen in die politischen Institutionen, in die Wissenschaft und in die Medien sind die Zusammenhänge weniger eindeutig. Unter den Personen, die keine Freiwilligenarbeit leisten, gibt es zwar leicht mehr Personen, die den politischen Institutionen, der Wissenschaft und den Medien misstrauen. Bei den Personen mit eher oder sehr hohem Vertrauen in die Institutionen findet man aber keine klaren Unterschiede zwischen formellen, informellen oder nicht Freiwilligen. Das Vertrauen in die politischen Institutionen hängt weniger mit Freiwilligkeit zusammen als vielmehr mit dem politischen Interesse und in geringerem Masse mit der politischen Partizipation. Das Interesse an der Politik und das Vertrauen in die politischen Institutionen gehen Hand in Hand. Am meisten Vertrauen haben Personen, die sich in der Mitte oder etwas links der Mitte einordnen. 

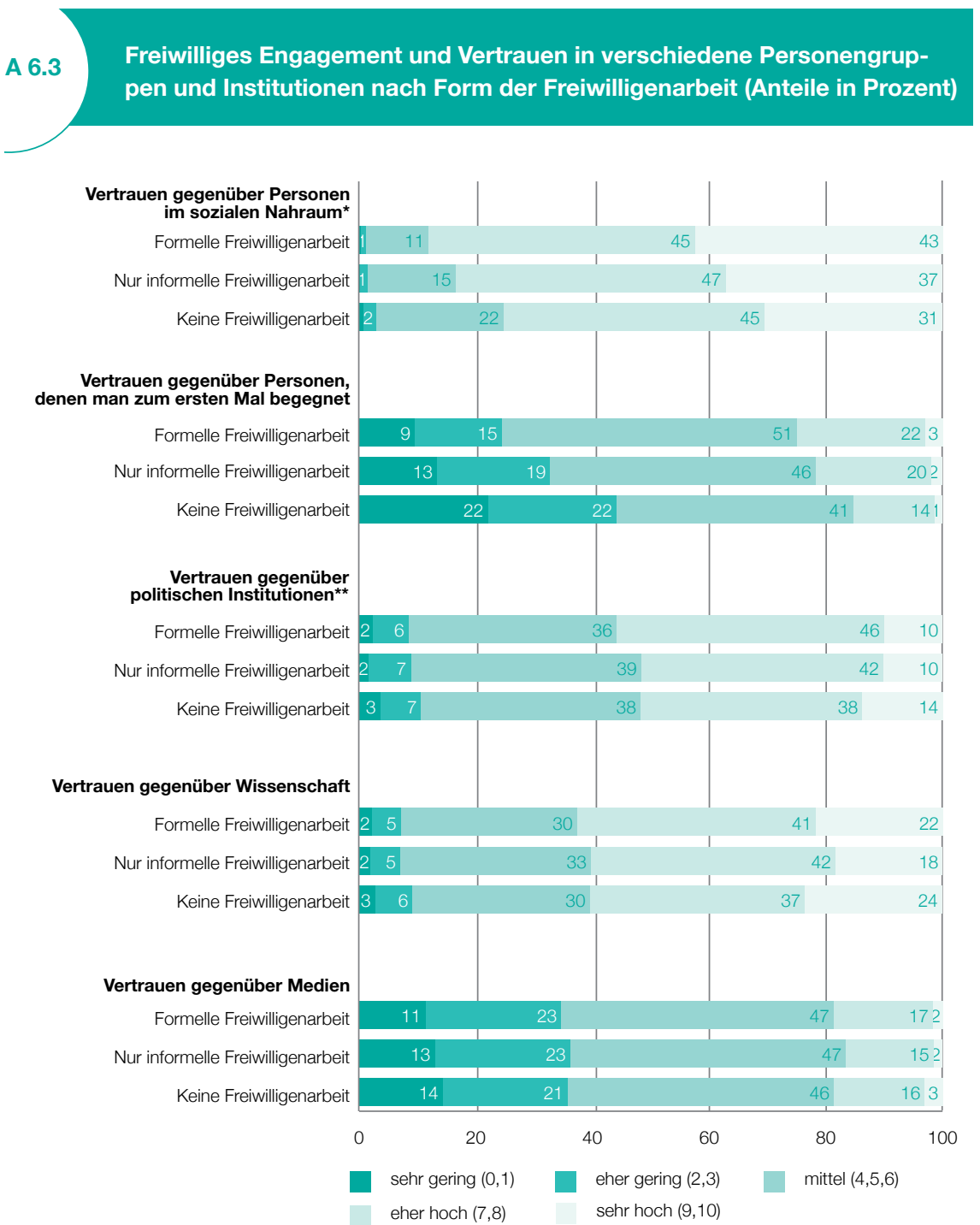

Anzahl Befragte: zwischen 4927 und 4941. Hinweise: * Zusammenfassender Index aus Vertrauen gegenüber Verwandten, Freundlnnen und Nachbarn. ${ }^{\star \star}$ Zusammenfassender Index aus Vertrauen gegenüber dem Bundesrat, dem nationalen Parlament, der kantonalen Regierung/Verwaltung, den Behörden in der Wohngemeinde und den Gerichten in der Schweiz. Lesebeispiel: 9 Prozent der Personen, die sich in einem Verein oder einer Organisation freiwillig engagieren, äussern ein sehr geringes Vertrauen gegenüber Personen, denen sie zum ersten Mal begegnen (Werte 0 oder 1). Bei Personen, die sich nur informell freiwillig engagieren sind es 13 Prozent und bei Personen ohne Freiwilligenarbeit sind es 22 Prozent. 


\section{Das Vertrauen in die Medien und in die Wissenschaft ist von anderen Faktoren abhängig}

Wenn man die verschiedenen Faktoren, die einen Einfluss auf das Vertrauen haben, in einem hier nicht dargestellten Mehrvariablen-Modell testet, so zeigt sich, dass das freiwillige Engagement einen signifikanten Einfluss auf das Vertrauen in Personen aus dem sozialen Nahraum und in fremde Personen hat. Daneben spielen aber auch Faktoren wie die Bildung, das politische Interesse sowie die Häufigkeit des Treffens mit anderen Personen eine wichtige Rolle. Auch das Vertrauen in die politischen Institutionen hängt mit dem freiwilligen Engagement zusammen, einen stärkeren Effekt haben hier jedoch die Bildung, das politische Interesse und - wie bereits angesprochen - die Nationalität. Das freiwillige Engagement hat dagegen keinen signifikanten Einfluss auf das Vertrauen in die Wissenschaft und in die Medien. Während beim Vertrauen in die Wissenschaft die Bildung, das politische Interesse und das Alter den stärksten Einfluss haben, sind beim Vertrauen in die Medien vor allem das politische Interesse sowie die politische Ausrichtung gemessen am Links-Rechts-Schema bedeutsam. Bei einer Ausrichtung auf die Mitte und bei einer eher linken Grundhaltung ist das Vertrauen in die Medien tendenziell grösser als bei einer rechten Grundhaltung.

Zusammenfassend kann man sagen, dass sich die Schweizer Bevölkerung nicht nur durch ein hohes freiwilliges Engagement auszeichnet, sondern auch durch eine relativ hohe politische Beteiligung und ein hohes Mass an Vertrauen. Ob die politische Beteiligung und das Vertrauen in die Mitmenschen sowie in die politischen und gesellschaftlichen Institutionen dazu führt, dass man sich eher freiwillig engagiert, oder ob umgekehrt das freiwillige Engagement dazu führt, dass man mehr Vertrauen gewinnt und sich eher politisch einbringt, kann hier nicht abschliessend beurteilt werden. Beides dürfte zu einem Teil richtig sein, und die Effekte verstärken sich wahrscheinlich gegenseitig. 



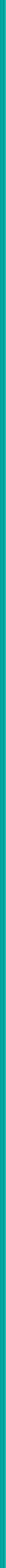


1 Freiwilligkeit

2 Formelle Freiwilligenarbeit

2 und Ehrenamt

3 Informelle Freiwilligenarbeit

4 Spenden und weitere Formen von Freiwilligkeit

5 Motive, Mobilisierung und Potenzial

(6)

Politisches Engagement, Vertrauen und die Freiwilligkeit

Untersuchungsmethode und Stichprobe

Literaturverzeichnis 



\section{Erhebungsmethode}

Der Monitor 2020 wurde in einem sequenziellen Web-Papier-Modus durchgeführt. Befragt wurde in den drei Landessprachen Deutsch, Französisch und Italienisch. Die Erhebung dauerte vom 11. April bis zum 26. August 2019.

Die Stichprobe wurde vom Bundesamt für Statistik aus dem Stichprobenrahmen für Personen- und Haushaltserhebungen (SRPH) gezogen. Die Zielpersonen erhielten ein Ankündigungsschreiben mit der Schweizerischen Gemeinnützigen Gesellschaft (SGG) als AbsenderIn. Das Ankündigungsschreiben war unterschrieben vom Präsidenten der SGG und dem Studienleiter und enthielt neben verschiedenen Informationen die Zugangsdaten zum individuellen Onlinefragebogen. Zur Information und Unterstützung der Befragten wurden eine Helpline eingerichtet sowie eine Mailadresse angegeben, über die man den schriftlichen Fragebogen bestellen konnte. Zudem wurde eine Informationswebsite aufgeschaltet, auf der man sich über den Freiwilligen-Monitor, die Trägerschaft sowie den Datenschutz informieren konnte. Diese Webseite enthielt auch eine FAQ zur Durchführung der Befragung.

Die Online-Befragung wurde im «responsive design» durchgeführt: Man konnte also die Befragung bequem auf unterschiedlichen Endgeräten (Desktop-PC, Laptop, Tabletcomputer, Smartphone) ausfüllen.

Wer nicht aufdas Ankündigungsschreiben reagierte, erhielt im Abstand von jeweils je drei Wochen zwei Erinnerungsschreiben. Mit dem zweiten Erinnerungsschreiben wurde zusätzlich ein schriftlicher Fragebogen mit einem frankierten Rückantwort-Couvert verschickt. Der Papierfragebogen umfasste 36 Seiten und wurde als $\mathrm{A}_{4}$-Broschüre gedruckt.

Bei der Erhebung wurde eng mit dem LINK Institut in Luzern zusammengearbeitet. LINK war unter der Leitung von Susanne Graf vorwiegend für die technischen Aspekte der Befragung verantwortlich: den Pretest, die Helpline, das Adressmanagement, die Programmierung des Fragebogens, das Hosting der Online-Befragung, das Dateneinlesen und die Datenaufbereitung sowie für Druck, Verpackung und Versand der Briefschaften. 


\section{Fragebogenentwicklung und Pretest}

Der Fragebogen wurde in Zusammenarbeit mit der Projektgruppe und unter Berücksichtigung der Anliegen der Partner auf der Grundlage der früheren Freiwilligen-Monitore weiterentwickelt und für die Online-Befragung optimiert. Die Übersetzungen des Fragebogens ins Französische und Italienische wurden vom Bundesamt für Statistik erstellt, sodass die Konkordanz mit der Schweizerischen Arbeitskräfteerhebung (SAKE) sichergestellt war.

Vom 12. bis 17. Dezember 2018 wurde ein Pretest mit 201 Testpersonen durchgeführt. Getestet wurden Programmablauf und Filterführung, Verständlichkeit der Fragestellungen, Vollständigkeit der Antwortvorgaben und Ausfülldauer. Grundsätzlich wurde das Thema als spannend eingeschätzt und der Fragebogen zwar als etwas lang, aber verständlich und übersichtlich taxiert. Die Online-Version funktionierte auf allen Geräten gut. Das Begleitmaterial (Ankündigungsschreiben, Internetseite und Informationen) sowie die Übersetzungen und die Gestaltung des schriftlichen Fragebogens erwiesen sich als hilfreich und zweckmässig. Im Anschluss an den Pretest mussten deshalb lediglich kleinere Anpassungen und Optimierungen vorgenommen werden.

\section{Grundgesamtheit, Stichprobenziehung, Ausschöpfung und Repräsentativität}

Die Grundgesamtheit des Freiwilligen-Monitors 2020 ist die ständige Wohnbevölkerung in der Schweiz im Alter ab 15 Jahren. Damit auch kleine Kantone angemessen in der Stichprobe vertreten sind, erfolgte die Stichprobenziehung durch das BFS geschichtet nach Kantonen mit einem leichten Oversampling der kleinen Kantone. Für die Auswertungen auf der Ebene der Gesamtschweiz wurde dieses Oversampling mit einer Gewichtung nach Kantonen sowie zusätzlich nach Geschlecht korrigiert. Die Stichprobe wurde in eine Basisstichprobe (22 290 Adressen) und eine Reservestichprobe (2 248 Adressen) unterteilt.

Insgesamt haben 5002 Personen den Fragebogen vollständig ausgefüllt: $3576(72 \%)$ online und $1426(28 \%)$ auf Papier. Bei 24 o8o zugestellten Ankündigungsbriefen (inkl. Reserve) ergibt dies eine Teilnahmequote von 20.8 Prozent. In der Romandie (Teilnahmequote: $23.0 \%$,) und in der italienischsprachigen 
Schweiz (21.8 \%) wurde eine bessere Ausschöpfung erreicht als in der Deutschschweiz (20.0\%). Die Teilnahmequote liegt etwas unter der Teilnahmequote von 2014 (24.6\%; in beiden Fällen wird die Bruttoausschöpfung verglichen), was auch daran lag, dass im Web-Papier-Modus keine telefonische Erinnerung vorgesehen war. Für das Ausfüllen des Online-Fragebogens benötigten die Befragten durchschnittlich 25 Minuten. 323 Personen bzw. 8 Prozent der Online-Antwortenden haben den Fragebogen nicht vollständig ausgefüllt. Diese «AbbrecherInnen» wurden aus den Daten entfernt.

Die Teilnehmenden repräsentieren die ständige Wohnbevölkerung in der Schweiz. Die verschiedenen Altersgruppen sind korrekt abgebildet. Neben der erwähnten Gewichtung zum Ausgleich des leichten Oversamplings der kleinen Kantone war nur eine kleine Verzerrung nach Geschlecht zu korrigieren (an der Befragung haben 53.4\% Frauen und $46.6 \%$ Männer teilgenommen).

Ein besonderes Augenmerk muss auf die Stichprobenausfälle wegen Sprachproblemen gerichtet werden, da es sich dabei nicht um «stichprobenneutrale» Ausfälle handelt. Die Migrationsbevölkerung, die keine der drei Landessprachen Deutsch, Französisch, Italienisch spricht, in denen die Fragen gestellt wurden, konnte an der Befragung nicht teilnehmen. Korrekterweise müsste deshalb von der «sprachassimilierten ständigen Wohnbevölkerung in der Schweiz» gesprochen werden. Wie in den früheren Monitoren müssen wir zudem davon ausgehen, dass insbesondere formell freiwillig engagierte Personen eher am Monitor teilnehmen. Vor diesem Hintergrund wurden die Ergebnisse mit der Schweizerischen Arbeitskräfteerhebung (SAKE) verglichen und validiert.

\section{Vergleich mit den früheren Monitoren}

Eine besondere Herausforderung bei den Freiwilligen-Monitoren besteht in den Vergleichen über die Zeit, da verschiedene Anpassungen an der Erhebungsmethode, an der Stichprobenziehung und am Fragebogen vorgenommen werden mussten. Die bisherigen Freiwilligen-Monitore beruhen auf folgenden Erhebungsmethoden: Bei den Freiwilligen-Monitoren 2007 und 2010 (Erhebungsjahre 2006 und 2009) handelt es sich um telefonische Befragungen (CATI: Computer Assisted Telephone Interviews). Der Freiwilligen-Monitor 
2016 (Erhebungsjahr 2014) wurde im Mixed-Mode durchgeführt. Die kontaktierten Personen konnten die Fragen entweder telefonisch beantworten (CATI) oder den Fragebogen online ausfüllen (CAWI: Computer Assisted Web Interviews). Erst für den vorliegenden Freiwilligen-Monitor 2020 (Erhebungsjahr 2019) wurde auf einen sequenziellen Web-Papier-Modus gewechselt. Die Basis für die Stichprobenziehung war in den ersten beiden Monitoren das Telefonverzeichnis von Swiss Directories. Ab 2014 wurde die Stichprobe vom Bundesamt für Statistik aus dem Stichprobenregister für Personen- und Haushaltserhebungen (SRPH) gezogen, das auf den Einwohnerregistern der Gemeinden und der Kantone beruht.

Die Anpassungen bei der Erhebungsmethode und bei der Stichprobenziehung erfolgten auch aufgrund der neuesten Entwicklungen im Telekommunikationsbereich und den Veränderungen im Kommunikationsverhalten der Bevölkerung. Immer weniger Personen in der Schweiz haben einen Festnetzanschluss und lassen ihre Telefonnummer registrieren. Vor allem viele junge Personen nutzen heute nur noch Mobiltelefone und sind weitaus weniger bereit, an telefonischen Befragungen in der Länge des FreiwilligenMonitors teilzunehmen. Gleichzeitig haben immer mehr Personen Zugang zum Internet, die früheren Alters- und Bildungsunterschiede bei der Internetnutzung haben sich weitgehend ausgeglichen.

Für die Zeitvergleiche haben die Methodenwechsel verschiedene Konsequenzen. Jede Erhebungsmethode hat ihre Vor- und Nachteile und bringt Anpassungen am Fragebogen mit sich (vgl. Jacob et al. 2019). Die grösste Herausforderung besteht darin, dass die Teilnahmechancen für verschiedene Bevölkerungsgruppen von der Erhebungsmethode abhängen können. Personen, die sehr engagiert sind und sich für ein Thema interessieren, machen eher bei Online-Befragungen mit. Weniger motivierte Personen lassen sich besser in einem Telefongespräch überzeugen, allerdings nur, wenn sie auch tatsächlich kontaktiert werden können und das Telefongespräch annehmen. Streng genommen erlauben die Methodenbrüche und die sich ändernde Zusammensetzung der Stichprobe keine direkten Vergleiche zwischen den verschiedenen Freiwilligen-Monitoren (vgl. auch Freitag et al. 2016,38). Trotzdem haben wir gewisse Zeitvergleiche vorgenommen, wobei die Ergebnisse jeweils für die verschiedenen Erhebungsarten getrennt dargestellt und mit der nötigen Zurückhaltung interpretiert wurden. 


\section{Analysen und Signifikanzen}

Obwohl der Freiwilligen-Monitor auf einer breiten Datenbasis beruht und die Daten nach streng wissenschaftlichen Kriterien erhoben und ausgewertet wurden, muss berücksichtigt werden, dass sich Stichprobenerhebungen stets innerhalb gewisser Fehlerspannen bewegen. Das bekannteste Mass zur statistischen Kontrolle dieser Fehlerspanne ist der so genannte Vertrauensbereich. Die Grösse des Vertrauensbereichs berechnet sich mit folgender Formel:

$$
\begin{aligned}
& V= \pm 2 \sqrt{\frac{p(100-p)}{n}} \\
& V=\text { Vertrauensbereich } \\
& \mathrm{p}=\text { Anteil der Befragten, die eine bestimmte Antwort gegeben haben (in Prozentpunkten) } \\
& \mathrm{n}=\text { ungewichtete Stichprobengrösse }
\end{aligned}
$$

Wenn also 39 Prozent der Befragten in der Stichprobe angeben, dass sie in den letzten vier Wochen formelle Freiwilligenarbeit geleistet haben, so liegt bei einer Stichprobengrösse von 5002 Personen der «wahre» Wert in der Grundgesamtheit mit einer Wahrscheinlichkeit von 95 Prozent zwischen 37.6. und 40.4 Prozent (Vertrauensbereich: \pm 1.4 Prozentpunkte).

Die dargestellten Ergebnisse wurden zusätzlich mit verschiedenen (multivariaten) Analysen abgesichert und auf statistische Signifikanz geprüft. Aus Platzgründen fand die Darstellung der entsprechenden Analysen keinen Eingang in den Text. 


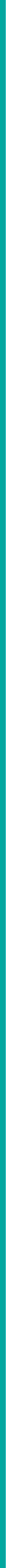


1 Freiwilligkeit

2 Formelle Freiwilligenarbeit

2 und Ehrenamt

3 Informelle Freiwilligenarbeit

4. Spenden und weitere Formen von Freiwilligkeit

5 Motive, Mobilisierung und Potenzial

(6)

Politisches Engagement, Vertrauen und die Freiwilligkeit

Untersuchungsmethode und Stichprobe

Literaturverzeichnis 

Ackermann, Maya, Kathrin Ackermann und Markus Freitag (2017): VereinsMonitor. Das ehrenamtliche Engagement in der Schweiz. Zürich: MigrosKulturprozent.

Ammann, Herbert (2001): Von Freiwilligkeit sei die Rede. Ein Vorschlag zur Klärung der Begriffe. Zürich: Schweizerische Gemeinnützige Gesellschaft. Ammann, Herbert (2008): Begrifflichkeiten und deren Auswirkung auf die Forschung am Beispiel des Freiwilligen-Monitors. In: Herbert Ammann, Raimund Hasse, Monika Jakobs und Gabriela Riemer-Kafka (Hrsg). Freiwilligkeit. Ursprünge, Erscheinungsformen, Perspektiven. Zürich: Seismo, S. 19-35. Ammann, Herbert (2011): «Forschung Freiwilligkeit» in der Schweiz. Ein nationaler Sonderweg mit internationaler Resonanz? In: Eckhard Priller, Mareike Alscher, Dietmar Dathe und Rudolf Speth (Hrsg.): Zivilengagement. Herausforderungen für Gesellschaft, Politik und Wissenschaft. Münster: Lit, S. $227-248$.

Ammann, Herbert, Ruth Bachmann und Riccarda Schaller (2004):Unternehmen unterstützen Freiwilligkeit. Zürich: Seismo.

Aregger, Doris (2012): Freiwillige Engagierte - Engagierte Freiwillige. Wer sind die Schweizer Freiwilligen und was leisten sie? Eine empirische Analyse der Determinanten der Freiwilligenarbeit in der Schweiz. Abhandlung zur Erlangung der Doktorwürde der Philosophischen Fakultät der UniversitätZürich. Schweiz. BFS (2015): Freiwilliges Engagement in der Schweiz 2013/14. Neuchâtel: Bundesamt für Statistik.

Bourdieu, Pierre (1998): Praktische Vernunft. Zur Theoriedes Handelns. Frankfurt am Main: Suhrkamp.

Braun, Sebastian (2003): Zwischen Gemeinschaftsorientierung und Selbstverwirklichung: Motive zu freiwilligem Engagement. In: Jürgen Baur und Sebastian Braun (Hrsg.) Integrationsleistungen von Sportvereinen als Freiwilligenorganisationen. Aachen: Meyer \& Meyer, S. 242-267.

Braun, Sebastian und Tina Nobis (Hrsg.) (2011): Migration, Integration und Sport: Zivilgesellschaft vor Ort. Wiesbaden: VS Verlag.

Bühlmann, Jacqueline und Beat Schmid (1999): Unbezahlt-aber trotzdem Arbeit: Zeitaufwand für Haus- und Familienarbeit, Ehrenamt, Freiwilligenarbeit und Nachbarschaftshilfe. Neuchâtel: Bundesamt für Statistik. 
Bühlmann, Marc und Markus Freitag (2007): Freiwilligkeit als soziales Kapital der Gesellschaft. Ein Vergleich der Schweizer Kantone. In: Peter Farago (Hrsg.): Freiwilliges Engagement in der Schweiz: Ergebnisse neuer Forschungen. Zürich: Seismo, S. 56-107.

Degen, Bernard (2010): Geschichte der NPO in der Schweiz. In: Bernd Helmig, Hans Lichtsteiner und Markus Gmür (Hrsg.): Der Dritte Sektor der Schweiz. Die Schweizer Länderstudie im Rahmen des Johns Hopkins Comparative Nonprofit Sector Project (CNP). Bern: Haupt, S. 59-97.

Eckhardt, Giana M. und Fleura Bardhi (2015): The sharing economy isn't about sharing at all. Harvard Business Review, 28. Januar 2015, https://hbr. $\mathrm{org} / 2015 / \mathrm{o} /$ the-sharing-economy-isnt-about-sharing-at-all [aufgerufen am 27.2.2020].

Farago, Peter (Hrsg.) (2007): Freiwilliges Engagement in der Schweiz. Zürich: Seismo.

Farago, Peter und Herbert Ammann (Hrsg.) (2006): Monetarisierung der Freiwilligkeit. Referate und Zusammenfassungen der 5 . Tagung der Freiwilligenuniversität vom 30 . bis 31.5.2005 in Luzern. Zürich: Seismo.

Freitag, Markus (Hrsg.) (2016): Das soziale Kapital der Schweiz. Zürich: Verlag Neue Zürcher Zeitung.

Freitag, Markus und Paul C. Bauer (2016): Was uns zusammenhält: Zwischenmenschliches Vertrauen als soziales Kapital in der Schweiz. In: Markus Freitag (Hrsg.): Das soziale Kapital der Schweiz. Zürich: Verlag Neue Zürcher Zeitung, S. 149-179.

Freitag, Markus, Anita Manatschal, Kathrin Ackermannund Maya Ackermann (2016): Freiwilligen-Monitor Schweiz 2016. Zürich: Seismo.

Freitag, Markus und Isabelle Stadelmann-Steffen (2011): Die Schweizer Gemeinden und ihre Vereine: Strukturen - Leistungen - Bewertungen. In: Herbert Ammann (Hrsg.): Grenzen-Los! Fokus Gemeinde: Freiwilliges Engagement in Deutschland, Österreich und der Schweiz. Referate der 2. Internationalen Vernetzungskonferenz 2010 in Rüschlikon. Zürich: Seismo, S. 35-66.

Haberkern, Klaus (2009): Pflege in Europa. Familie und Wohlfahrtsstaat. Wiesbaden: VS Verlag für Sozialwissenschaften.

Jacob, Rüdiger, Andreas Heinz und Jean Philippe Décieux (2019): Umfrage. Einführung in die Methoden der Umfrageforschung. Berlin: De Gruyter. 
Ladner, Andreas, Reto Steiner, Katia Horber-Papazian, Julien Fiechter, Caroline Jacot-Descombes, Claire Kaiser (2013): Gemeindemonitoring 2009/2010. Bericht zur fünften gesamtschweizerischen Gemeindeschreiberbefragung. Bern: KPM-Verlag.

Lamprecht, Markus, Adrian Fischer, Rahel Bürgi und Hanspeter Stamm (2018): Vertrauens-Monitor. Gesellschaftliches Engagement und Vertrauen. Zürich: Migros-Kulturprozent.

Lamprecht, Markus, Adrian Fischer und Hanspeter Stamm (2011): Die Schweizer Sportvereine. Strukturen, Leistungen, Herausforderungen. Zürich: Seismo.

Linder, Wolf (2012): Schweizerische Demokratie. Institutionen, Prozesse, Perspektiven. Bern: Haupt.

Müller, Andreas (Hrsg.) (2015): Bürgerstaat und Staatsbürger. Milizpolitikzwischen Mythos und Moderne. Zürich: Verlag Neue Zürcher Zeitung.

Mutz, Michael und Ulrike Burrmann (2015): Zur Beteiligung junger Migrantinnen und Migranten am Vereinssport. In: Ulrike Burrmann, Michael Mutz und Ursula Zender (Hrsg.) Jugend, Migration und Sport. Kulturelle Unterschiede und die Sozialisation zum Vereinssport. Wiesbaden: Springer. S. 69-90.

Nadai, Eva (1996): Gemeinsinn und Eigennutz. Freiwilliges Engagement im Sozialbereich. Bern: Haupt.

Nadai, Eva (2004): Begrifflichkeit im Themenfeld Freiwilligenarbeit. In: Guido Münzel, Sandro Guzzi Heeb, Bernadette Kadishi, Eva Nadai und Jacqueline Schön-Bühlmann (2004): Bericht zur Freiwilligenarbeit in der Schweiz. Neuchâtel: Bundesamt für Statistik., S. 16-34.

Nollert, Michael und Christian Huser (2007): Freiwillig Aktive in der Schweiz: Einflussfaktoren und typische Profile. In: Peter Farago (Hrsg.): Freiwilliges Engagement in der Schweiz. Zürich: Seismo, S. 14-55.

Priller, Eckhard, Mareike Alscher, Dietmar Dathe und Rudolf Speth (Hrsg.) (2011): Zivilengagement. Herausforderungen für Gesellschaft, Politik und Wissenschaft. Münster: Lit.

Putnam, Robert D. (1995): Bowling alone: America's declining social capital. Journal of Democracy 6(1), S. 65-78.

Putnam, Robert D. (2000): Bowling alone: The collapse and revival of American community. New York: Simon \& Schuster. 
Samochowiec, Jakub, Leonie Thalmann und Andreas Müller (2018): Die neuen Freiwilligen-DieZukunftzivilgesellschaftlicherPartizipation. Rüschlikon: GDI Gottlieb Duttweiler Institut.

Schön-Bühlmann, Jacqueline (2008): Freiwilligenarbeit in der Schweiz. Neuchâtel: Bundesamt für Statistik.

Schön-Bühlmann, Jacqueline (2011): Freiwilligenarbeit in der Schweiz: Unterschiede zwischen ausgewählten regionalen Gliederungen. Neuenburg: BFS aktuell, Bundesamt für Statistik.

Schüll, Peter (2006): Motive und Erwartungen von Freiwilligen. Eine Ernüchterung für Monetarsierungsbefürworter? In: Peter Farago und Herbert Ammann (Hrsg.): Monetarisierung der Freiwilligkeit. Referate und Zusammenfassungen der 5. Tagung der Freiwilligenuniversität vom 30. bis 31.5.2005 in Luzern. Zürich: Seismo, S. 310-325.

Schumacher, Beatrice, (Hrsg.) (2010). Freiwilligverpflichtet. Gemeinnütziges Denken und Handeln in der Schweiz seit 180o. Zürich: Verlag Neue Zürcher Zeitung. Simonson, Julia, Claudia Vogel und Clemens Tesch-Römer (Hrsg.) (2016): Freiwilliges Engagement in Deutschland. Der Deutsche Freiwilligensurvey 2014. Berlin: Deutsches Zentrum für Altersfragen.

Stadelmann-Steffen, Isabelle, Markus Freitag und Marc Bühlmann (2007): Freiwilligen-Monitor Schweiz 2007. Zürich: Seismo.

Stadelmann-Steffen, Isabelle, Richard Traunmüller, Birte Gundelach und Markus Freitag (2010): Freiwilligen-Monitor Schweiz 2010. Zürich: Seismo.

Stokar, Thomas von, Martin Peter, Remo Zandonella, Vanessa Angst, Kurt Pärli, Gabi Hildesheimer, Johannes Scherrer, Wilhelm Schmid (2018): Sharing Economy - teilen statt besitzen. Zürich: vdf Hochschulverlag.

Tocqueville, Alexis de (2016 [1835]): Über die Demokratie in Amerika. Stuttgart: Reclam.

Traunmüller, Richard, Isabelle Stadelmann-Steffen, Kathrin Ackermann und Markus Freitag (2012): Zivilgesellschaft in der Schweiz: Analysen zum Vereinsengagement auf lokaler Ebene. Zürich: Seismo.

Vogel, Claudia und Clemens Tesch-Römer (2016): Informelle Unterstützung ausserhalb des Engagements: Instrumentelle Hilfen, Kinderbetreuung und Pflege im sozialen Nahraum. In: Julia Simonson, Claudia Vogel und Clemens Tesch-Römer (Hrsg.): Freiwilliges Engagement in Deutschland. Der Deutsche Freiwilligensurvey 2014. Berlin: Deutsches Zentrum für Altersfragen, S. 251-280. 
Wehner, Theo und Stefan T. Güntert (Hrsg.) (2015): Psychologie der Freiwilligenarbeit. Motivation, Gestaltung und Organisation. Berlin, Heidelberg: Springer.

Wilson, John (2000): Volunteering. Annual Review of Sociology 26(1), S. 215-240. 



\section{Dank}

Der Freiwilligen-Monitor Schweiz 202o ist ein Gemeinschaftswerk zahlreicher Personen, Gremien und Organisationen, denen ein herzliches «Dankeschön» gebührt.

Zuallererst möchten wir uns bei den über 5 ooo Personen herzlich bedanken, die sich die Zeit und die Mühe genommen haben, die Fragen zum freiwilligen Engagement zu beantworten. Ohne ihren freiwilligen Einsatz wäre diese Studie nicht möglich geworden.

Die Schweizerische Gemeinnützige Gesellschaft (SGG) hat im UNO-Jahr der Freiwilligen anno 2001 entschieden, die Freiwilligenarbeit der Schweiz langfristig zu erforschen und zu fördern. Seither hat die SGG über fünf Millionen Franken in die Förderung der Freiwilligenarbeit in der Schweiz investiert.

Die Mitglieder der SGG-Kommission Forschung Freiwilligkeit (KFF) unter der Leitung von Peter Farago sowie deren „Projektgruppe Freiwilligen-Monitor“ haben alle vier Freiwilligen-Monitore geplant und begleitet.

Jacqueline Schön-Bühlmann vom Bundesamt für Statistik (BFS) hat seit Beginn dafür gesorgt, dass das BFS für den Freiwilligen-Monitor jeweils die Stichproben zieht und für die Monitor-Befragung die bestmöglichen und aktuellsten Adressen zur Verfügung stellt.

Migros-Kulturprozent und die Beisheim Stiftung haben diesen Monitor ideell und finanziell zusammen mit der SGG gefördert. Und weitere zo Organisationen haben die Entwicklung und Herausgabe des Monitors als «Partner Resultate»* oder als «Partner Mitsprache»** unterstützt.

Die wissenschaftliche Forschungsarbeit dieses Monitors leisteten Markus Lamprecht, Adrian Fischer sowie Hanspeter Stamm von Lamprecht \& Stamm Sozialforschung und Beratung. Die Befragung bei den über 5000 Personen wurde vom LINK Institut durchgeführt.

Franziska Dörig vom Seismo Verlag fördert die Buchserie «Freiwilligkeit», in der auch der vierte Freiwilligen-Monitor Schweiz erscheinen darf - zum 
ersten Mal auch auf Französisch sowie als PDF zum Herunterladen. Die französische Übersetzung erfolgte durch Irène Stumm.

Ihnen allen gilt ein herzlicher Dank, merci beaucoup, grazie mille, grazia fitg!

Lukas Niederberger

Geschäftsleiter SGG

* «Partner Resultate» des Freiwilligen-Monitors erhalten vertiefte Analysen des Monitors für ihre Wirkungsbereiche und können vertiefte Analysen der Monitordaten in Auftrag geben. Folgende Organisationen sind «Monitor-Partner Resultate»: Bundesamt für Sport BASPO, Die dargebotene Hand Schweiz, Evangelisch-reformierte Kirche Schweiz EKS, Förderverein Nachbarschaftshilfe Zürich, Gemeinnützige Gesellschaft der Stadt Luzern, Kanton Schaffhausen (Departement des Innern), Kanton Uri (Amt für Soziales), Kanton Zürich (Sicherheitsdirektion), Ökonomische Gemeinnützige Gesellschaft des Kantons Bern OGG, Procap Schweiz, Schweizerischer Arbeitgeberverband, Schweizerischer Städteverband SSV, Schweizerisches Rotes Kreuz SRK.

** «Partner Mitsprache» des Freiwilligen-Monitors geniessen die Vorteile der «Partner Resultate» und können zusätzlich ihre Fragen und Themen in die Monitor-Befragung einbringen. Es sind die folgenden Organisationen: Benevol Schweiz, Caritas Schweiz, Dachverband Schweizer Jugendparlamente DSJ, Gemeinnützige Gesellschaft des Kantons St. Gallen GGKSG, Kanton Zug (Direktion des Innern), Pro Juventute Schweiz, Pro Senectute Schweiz, Römisch-katholische Zentralkonferenz RKZ, Schweizerische Arbeitsgemeinschaft der Jugendverbände SAJV, Schweizerische Gemeinnützige Frauen SGF, Schweizerischer Gemeindeverband SGV, Schweizerisches Rotes Kreuz SRK, Stadt Zürich (Gesundheits- und Umweltdepartement), Stiftung Mercator Schweiz. 
Die Soziologen Markus Lamprecht, Adrian Fischer und Hanspeter Stamm arbeiten für das Sozialforschungsbüro Lamprecht \& Stamm, das seit 1993 für verschiedene Bundesämter, kantonale und lokale Stellen sowie für öffentliche und private Organisationen tätig ist. Zu ihren Forschungsschwerpunkten gehören Sport, Freizeit, Vereine, Gesundheit und soziale Ungleichheit. Aus ihrer Arbeit sind zahlreiche Artikel in der Fach- und Tagespresse sowie verschiedene Bücher entstanden. Im Seismo Verlag erschienen «Die soziale Ordnung der Freizeit» (1994), "Sport zwischen Kultur, Kult und Kommerz» (2002), "Soziale Ungleichheiten in der Schweiz» (2003) und «Die Schweizer Sportvereine» (2012).

Der Freiwilligen-Monitor Schweiz 2020 informiert über den aktuellen Stand des unbezahlten gesellschaftlichen Engagements. In der Schweiz wird der freiwillige Einsatz zum Wohl von Mensch, Gesellschaft und Umwelt mit einer landesweiten Befragung regelmässig erforscht. Der Freiwilligen-Monitor Schweiz bringt in Erfahrung, warum sich Personen inmitten einer monetär geprägten Welt ohne Lohn fürs Gemeinwohl einsetzen und so den Zusammenhalt und die Lebendigkeit der Gesellschaft fördern. Der Freiwilligen-Monitor zeigt Trends und Entwicklungen der Freiwilligenarbeit auf und bietet dadurch wichtige Grundlagen für das Freiwilligen-Management in Verbänden und Vereinen, Heimen, Kirchen und Hilfswerken. Die im Monitor aufgezeigte Entwicklung des freiwilligen Engagements ist gleichzeitig auch ein Gradmesser für die Lebendigkeit sozialer Beziehungen und die Gemeinwohlorientierung in der Schweiz. Die Förderung des gesellschaftlichen Zusammenhalts und des freiwilligen Engagements bildet die Kernaufgabe der Schweizerischen Gemeinnützigen Gesellschaft (SGG). Die SGG hat den FreiwilligenMonitor Schweiz vor bald 20 Jahren initiiert und gibt inn alle vier bis fünf Jahre heraus.

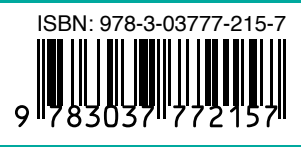

